\title{
Pacific
}

Journal of

Mathematics

\section{DEGENERATE TWO-BOUNDARY CENTRALIZER ALGEBRAS}

\section{ZAJJ DAUGHERTY}




\title{
DEGENERATE TWO-BOUNDARY CENTRALIZER ALGEBRAS
}

\author{
ZAJJ DAUGHERTY
}

Diagram algebras (for example, graded braid groups, Hecke algebras and Brauer algebras) arise as tensor power centralizer algebras, algebras of commuting operators for a Lie algebra action on a tensor space. This work explores centralizers of the action of a complex reductive Lie algebra $\mathfrak{g}$ on tensor space of the form $M \otimes N \otimes V^{\otimes k}$. We define the degenerate twoboundary braid algebra $\varphi_{k}$ and show that centralizer algebras contain quotients of this algebra in a general setting. As an example, we study in detail the combinatorics of special cases corresponding to Lie algebras $\mathfrak{g l}_{n}$ and $\mathfrak{s l}_{n}$ and modules $M$ and $N$ indexed by rectangular partitions. For this setting, we define the degenerate extended two-boundary Hecke algebra $\mathcal{H}_{k}^{\text {ext }}$ as a quotient of $\varphi_{k}$, and show that a quotient of $\mathscr{H}_{k}^{\text {ext }}$ is isomorphic to a large subalgebra of the centralizer. We further study the representation theory of $\mathcal{H}_{k}^{\text {ext }}$ to find that the seminormal representations are indexed by a known family of partitions. The bases for the resulting modules are given by paths in a lattice of partitions, and the action of $\mathcal{K}_{k}^{\text {ext }}$ is given by combinatorial formulas.

1. Introduction 91

2. The degenerate two-boundary braid algebra 93

3. The degenerate two-boundary Hecke algebra 98

4. Tensor space as a $\mathscr{H}_{k}^{\text {ext }}$-module 106

5. Seminormal representations of $\mathscr{H}_{k}^{\text {ext }} \quad 125$

Acknowledgments 141

References 141

\section{Introduction}

The phenomenon now known as Schur-Weyl duality was first studied by Frobenius and Schur in their work connecting the representation theory of the symmetric groups and the general linear groups. It has since stimulated many advances in

Supported by the National Science Foundation under grant number 0353038.

MSC2010: primary 20C08; secondary 05E10, 17B10.

Keywords: representation theory, combinatorics, Lie theory, Hecke algebras, braid groups, diagram algebras, tantalizers, Schur-Weyl duality. 
the development of tensor power centralizer algebras, algebras of operators which preserve symmetries in a tensor space. Striking examples include these:

(1) The Brauer algebras in [Brauer 1937] centralize the action of symplectic and orthogonal groups on tensor space $\left(\mathbb{C}^{n}\right)^{\otimes k}$.

(2) The graded Hecke algebra of type A centralizes the action of $\mathfrak{s l}_{n}$ on $L(\lambda) \otimes$ $\left(\mathbb{C}^{n}\right)^{\otimes k}$, where $L(\lambda)$ is the irreducible $\mathfrak{s l}_{n}$ module indexed by a partition $\lambda$; see [Arakawa and Suzuki 1998].

(3) The degenerate affine Wenzl algebra in [Nazarov 1996] centralizes the action of symplectic and orthogonal groups on $L(\lambda) \otimes\left(\mathbb{C}^{n}\right)^{\otimes k}$.

Orellana and Ram [2007] give a unified approach to studying tensor power centralizer algebras, including the affine and cyclotomic Hecke and Birman-MurakamiWenzl algebras.

Gier and Nichols [2009] uncovered in the study of loop models and spin chains in statistical mechanics yet another potential use of Schur-Weyl duality. They discovered a connection between the two-boundary Temperley-Lieb algebra and a quotient of the affine Hecke algebra of type C. The Temperley-Lieb algebra is the centralizer of the quantum group ${U_{q}}_{\mathfrak{s l}_{2}}$ on tensor space $M \otimes N \otimes\left(\mathbb{C}^{2}\right)^{\otimes k}$, where $M$ and $N$ are simple $U_{q} \mathfrak{s l}_{2}$-modules, which suggested the possibility of constructing affine Hecke algebra type $\mathrm{C}$ modules explicitly using Schur-Weyl duality tools.

In Section 2, we begin studying the centralizer of the action of $\mathfrak{g}$ on $M \otimes N \otimes V^{\otimes k}$, where $\mathfrak{g}$ is a finite-dimensional complex reductive Lie algebra and $M, N$, and $V$ are finite-dimensional irreducible $\mathfrak{g}$-modules. We define the degenerate two-boundary braid algebra $\mathscr{G}_{k}$, an associative algebra over the complex numbers. This braid algebra can be pictured as the degeneration of the quantum group analog, group algebra of the braid group in a space with two punctures, a generalization of the affine braid group studied in [Orellana and Ram 2007]. The algebra $\mathscr{G}_{k}$ is generated by

$$
\mathbb{C}\left[x_{1}, \ldots, x_{k}\right], \mathbb{C}\left[y_{1}, \ldots, y_{k}\right], \mathbb{C}\left[z_{0}, z_{1}, \ldots, z_{k}\right], \text { and } \mathbb{C} S_{k},
$$

with relations twisting the polynomial rings and the symmetric group together. The first main theorem, Theorem 2.1, is that $\varphi_{k}$ acts on $M \otimes N \otimes V^{\otimes k}$ and that this action commutes with the action of $\mathfrak{g}$. In many cases, both historic and new, this action will produce $\operatorname{End}_{\mathfrak{g}}\left(M \otimes N \otimes V^{\otimes k}\right)$. For example,

(i) when $\mathfrak{g}=\mathfrak{g l}_{n}$ or $\mathfrak{s l}_{n}, V$ is the standard representation, and

(a) $M$ and $N$ are trivial, the image of $\varphi_{k}$ in $\operatorname{End}_{\mathfrak{g}}\left(M \otimes N \otimes V^{\otimes k}\right)$ is the same as that of the symmetric group $S_{k}$;

(b) $M$ is trivial and $N$ is a simple highest weight module, the image of $\varphi_{k}$ in $\operatorname{End}_{\mathfrak{g}}\left(M \otimes N \otimes V^{\otimes k}\right)$ is the same as that of the graded Hecke algebra of type A; 
(ii) when $\mathfrak{g}=\mathfrak{s o}_{n}$ or $\mathfrak{s p}_{2 n}, V$ is the standard representation, and

(a) $M$ and $N$ are trivial, the image of $\varphi_{k}$ in $\operatorname{End}_{\mathfrak{g}}\left(M \otimes N \otimes V^{\otimes k}\right)$ is the same as that of the Brauer algebras;

(b) $M$ is trivial and $N$ is a simple highest weight module, the image of $\varphi_{k}$ in $\operatorname{End}_{\mathfrak{g}}\left(M \otimes N \otimes V^{\otimes k}\right)$ is the same as that of the degenerate affine Wenzl algebra.

We discuss the specifics of these examples in Remark 2.2.

In Section 3, we consider the new cases where $\mathfrak{g}=\mathfrak{s l}_{n}$ or $\mathfrak{g l}_{n}, M=L\left(\left(a^{p}\right)\right)$ (the finite-dimensional irreducible $\mathfrak{g}$-module indexed by the rectangular partition with $p$ parts of length $a), N=\left(\left(b^{q}\right)\right)$, and $V$ is the standard representation. Theorem 4.9 states that a twist of the representation given in Theorem 2.1 factors through this quotient. We call this quotient of $\varphi_{k}$ the extended degenerate two-boundary Hecke algebra $\mathscr{H}_{k}^{\text {ext }}$.

We further study the representation theory of $\mathscr{H}_{k}^{\text {ext }}$ throughout Sections 4 and 5, classifying the seminormal representations. Using the combinatorics of Young tableaux, we describe these representations explicitly in Sections 4.3 and 5. The basis elements for the resulting modules are given by paths in a lattice of partitions, and the action of $\mathcal{H}_{k}^{\text {ext }}$ is given in terms of contents of boxes in those partitions.

This work may proceed in a number of directions. First, an analogous theory may also be developed for centralizers of type B, C, and D, which will parallel that of the degenerate affine Wenzl algebra as studied in [Nazarov 1996; Ariki et al. 2006]. Also, functorial techniques developed in [Orellana and Ram 2007] may be used to promote the study of calibrated $\mathscr{H}_{k}^{\text {ext }}$-modules, given in Section 5, to that of all standard modules. This should extend to the study of standard modules for types B, C, and D as well.

Finally, one subalgebra of $\mathscr{H}_{k}^{\text {ext }}$, the degenerate two-boundary Hecke algebra $\mathscr{H}_{k}$, is of particular interest since it is strikingly similar to the graded Hecke algebra of type $\mathrm{C}$. This can be seen through the combinatorics that are presented throughout Sections 4.3 and 5 and in the action of the type $C$ Weyl group in the final proof of the paper. This observation suggests the possibility of studying representations of type C Hecke algebras using Schur-Weyl duality techniques, a study that is further developed in forthcoming papers.

\section{The degenerate two-boundary braid algebra}

Fix $k \in \mathbb{Z}_{\geq 0}$. Let $S_{k}$ be the symmetric group, which is generated by simple transpositions $s_{i}=(i i+1)$ and braid relations

$$
s_{i} s_{i+1} s_{i}=s_{i+1} s_{i} s_{i+1} \quad \text { and } \quad s_{i} s_{j}=s_{j} s_{i} \quad \text { if } j \neq i \pm 1 .
$$


The main object of study in this section is the degenerate two-boundary braid algebra, denoted $\mathscr{G}_{k}$, and is a two-boundary analog to the degenerate one-boundary braid algebra in [Daugherty et al. 2011]. By design, we will see in the Section 2.1 that $\varphi_{k}$ acts on tensor space of a specific form for a finite-dimensional reductive Lie algebra. The algebra $\mathscr{G}_{k}$ is generated over $\mathbb{C}$ by

$$
t_{\mathrm{w}} \quad \text { for } \mathrm{w} \in S_{k}, \quad x_{1}, \ldots, x_{k}, \quad y_{1}, \ldots, y_{k}, \quad \text { and } z_{0}, z_{1}, \ldots, z_{k},
$$

subject to relations as follows. Let $m_{1}=0$ and, for $j>1$, define $m_{j}=\sum_{1 \leq i<j} m_{i, j}$, where

$$
\begin{aligned}
m_{j-1, j} & =x_{j}-t_{s_{j-1}} x_{j-1} t_{s_{j-1}} & & \text { and } \\
m_{i, j} & =t_{(i j-1)} m_{j-1, j} t_{(i j-1)} & & \text { for } 1 \leq i<j-1 .
\end{aligned}
$$

Then $\mathscr{G}_{k}$ is the associative algebra generated over $\mathbb{C}$ by elements (2-2) with relations

$$
\begin{aligned}
& z_{i}=x_{i}+y_{i}-m_{i} \quad \text { for } 1 \leq i \leq k, \\
& t_{\mathrm{w}} t_{\mathrm{w}^{\prime}}=t_{\mathrm{ww}^{\prime}} \quad \text { for } \mathrm{w} \in S_{k} \\
& x_{i} x_{j}=x_{j} x_{i}, \quad y_{i} y_{j}=y_{j} y_{i}, \quad z_{i} z_{j}=z_{j} z_{i} \quad \text { for all admissible } i, j, \\
& t_{s_{i}} x_{j}=x_{j} t_{s_{i}}, \quad t_{s_{i}} y_{j}=y_{j} t_{s_{i}}, \quad t_{s_{i}} z_{j}=z_{j} t_{s_{i}} \quad \text { for } j \neq i, i+1, \\
& \left(z_{0}+\cdots+z_{i}\right) x_{j}=x_{j}\left(z_{0}+\cdots+z_{i}\right), \\
& \left(z_{0}+\cdots+z_{i}\right) y_{j}=y_{j}\left(z_{0}+\cdots+z_{i}\right) \quad \text { for } i \geq j, \\
& t_{s_{i}}\left(x_{i}+x_{i+1}\right)=\left(x_{i}+x_{i+1}\right) t_{s_{i}}, \quad \text { for } 1 \leq i \leq k-1, \\
& t_{s_{i}}\left(y_{i}+y_{i+1}\right)=\left(y_{i}+y_{i+1}\right) t_{s_{i}} \quad \text { for } \\
& \left(t_{s_{i}} t_{s_{i+1}}\right)\left(x_{i+1}-t_{s_{i}} x_{i} t_{s_{i}}\right)\left(t_{s_{i+i}} t_{s_{i}}\right)=x_{i+2}-t_{s_{i+1}} x_{i+1} t_{s_{i+1}}, \\
& \left(t_{s_{i}} t_{s_{i+1}}\right)\left(y_{i+1}-t_{s_{i}} y_{i} t_{s_{i}}\right)\left(t_{s_{i+i}} t_{s_{i}}\right)=y_{i+2}-t_{s_{i+1}} y_{i+1} t_{s_{i+1}} \\
& \quad \text { for } 1 \leq i \leq k-2,
\end{aligned}
$$

(2-11) $\quad x_{i+1}-t_{s_{i}} x_{i} t_{s_{i}}=y_{i+1}-t_{s_{i}} y_{i} t_{s_{i}}$ for $1 \leq i \leq k-1$.

2.1. Action on tensor space. Let $\mathfrak{g}$ be a finite-dimensional complex reductive Lie algebra. We fix a triangular decomposition

$$
\mathfrak{g}=\mathfrak{n}^{-} \oplus \mathfrak{h} \oplus \mathfrak{n}^{+}, \quad \text { with } \mathfrak{n}^{+}=\bigoplus_{\alpha \in R^{+}} \mathfrak{g}_{\alpha},
$$

and let $R^{+}$be a fixed set of positive roots for $\mathfrak{g}$. A weight is an element of $\mathfrak{h}^{*}=\operatorname{Hom}(\mathfrak{h}, \mathbb{C})$.

The trace form $\langle\cdot, \cdot\rangle: \mathfrak{g} \otimes \mathfrak{g} \rightarrow \mathbb{C}$ associated to a faithful representation $\theta$ of $\mathfrak{g}$ is defined by

$$
\langle x, y\rangle=\operatorname{tr}(\theta(x) \theta(y)) .
$$


This is an ad-invariant, symmetric, bilinear form that is nondegenerate on both $\mathfrak{g}$ and $\mathfrak{h}$. Therefore the map $\mathfrak{h} \rightarrow \mathfrak{h}^{*}$ defined by $h \mapsto\langle h, \cdot\rangle$ and $h_{\mu} \mapsto \mu$ is an isomorphism, where $h_{\mu}$ is the unique element of $\mathfrak{h}$ such that $\left\langle h_{\mu}, h\right\rangle=\mu(h)$ for all $h \in \mathfrak{h}$. Define the symmetric, bilinear, nondegenerate form $\langle\cdot, \cdot\rangle: \mathfrak{h}^{*} \otimes \mathfrak{h}^{*} \rightarrow \mathbb{C}$ by $\langle\lambda, \mu\rangle=\left\langle h_{\lambda}, h_{\mu}\right\rangle$.

Let $M, N$, and $V$ be finite-dimensional simple $\mathfrak{g}$-modules, and consider the action of $\mathfrak{g}$ on the tensor space $M \otimes N \otimes V^{\otimes k}$. Denote the centralizer of the action of $\mathfrak{g}$ on a $\mathfrak{g}$-module $U$ by

$$
\operatorname{End}_{\mathfrak{g}}(U)=\{\varphi \in \operatorname{End}(U) \mid x \varphi=\varphi x \text { for all } x \in \mathfrak{g}\} .
$$

We will construct a homomorphism $\Phi: \varphi_{k} \rightarrow \operatorname{End}_{\mathfrak{g}}\left(M \otimes N \otimes V^{\otimes k}\right)$ using the observation that the map given by

$$
\operatorname{End}_{\mathfrak{g}}(U) \rightarrow \operatorname{End}_{\mathfrak{g}}\left(U \otimes U^{\prime}\right), \quad \varphi \mapsto \varphi \otimes \operatorname{id}_{U^{\prime}}
$$

is an injective algebra homomorphism for any $\mathfrak{g}$-modules $U$ and $U^{\prime}$.

Fix a basis $\left\{b_{i}\right\}$ for $\mathfrak{g}$ and let $\left\{b_{i}^{*}\right\}$ be the dual basis to $\left\{b_{i}\right\}$ with respect to $\langle\cdot, \cdot\rangle$. The Casimir element of the enveloping algebra $\mathcal{U} \mathfrak{g}$ is

$$
\kappa=\sum_{i} b_{i} b_{i}^{*}
$$

and is central in $\cup \mathfrak{g}$. If $U$ and $U^{\prime}$ are $\mathfrak{g}$-modules, then $\kappa$ acts on $U \otimes U^{\prime}$ by

$$
\kappa \otimes \mathrm{id}_{U^{\prime}}+\mathrm{id}_{U} \otimes \kappa+2 \gamma, \quad \text { where } \gamma=\sum_{i} b_{i} \otimes b_{i}^{*} .
$$

Since $\kappa$ and $\gamma$ are independent of the choice of the basis, we have, in particular,

$$
\gamma=\sum_{i} b_{i}^{*} \otimes b_{i}
$$

Let $\gamma_{j, j^{\prime}}$ be the operator given by the action of $\gamma$ on the $j$ and $j^{\prime}$ factors of $V^{\otimes k}$ (acting by the identity on all other factors). Note that $\gamma_{j, j^{\prime}}=\gamma_{j^{\prime}, j}$ because of (2-15b). Similarly, for a factor $X(X=M, N, M \otimes N$, or $V$, where applied), we use the following notation:

$\gamma_{M, N} \quad \gamma$ acting on factors $M$ and $N$ in a tensor space,

$\gamma_{X, i} \quad \gamma$ acting on factor $X$ and the $i^{\text {th }}$ copy of $V$ in a tensor space,

$\kappa_{X} \quad \kappa$ acting on the factor $X$ in a tensor space,

$\kappa_{X, \leq j} \quad \kappa$ acting on the factor $X$ and the first $j$ factors of $V$, where $\kappa_{X, \leq 0}=\kappa_{X}$. 
Using (2-15) to apply $\kappa$ iteratively to $M \otimes V^{\otimes k}, N \otimes V^{\otimes k}$, and $M \otimes N \otimes V^{\otimes k}$, we find that as operators on $M \otimes N \otimes V^{\otimes k}$, for $X=M, N$ or $M \otimes N$,

$$
\kappa_{X, \leq j}=\kappa_{X}+j \kappa_{V}+2\left(\sum_{1 \leq i \leq j} \gamma_{X, i}+\sum_{1 \leq r<s \leq j} \gamma_{r, s}\right) .
$$

Theorem 2.1. There is an algebra homomorphism

$$
\Phi: \mathscr{G}_{k} \rightarrow \operatorname{End}_{\mathfrak{g}}\left(M \otimes N \otimes V^{\otimes k}\right)
$$

defined by

$$
\begin{array}{ll}
\Phi\left(x_{i}\right)=\frac{1}{2}\left(\kappa_{M, \leq i}-\kappa_{M, \leq i-1}\right), \quad & \Phi\left(y_{i}\right)=\frac{1}{2}\left(\kappa_{N, \leq i}-\kappa_{N, \leq i-1}\right), \\
\Phi\left(z_{i}\right)=\frac{1}{2}\left(\kappa_{M \otimes N, \leq i}-\kappa_{M \otimes N, \leq i-1}+\kappa_{V}\right) & \text { for } 1 \leq i \leq k, \\
\Phi\left(z_{0}\right)=\frac{1}{2}\left(\kappa_{M \otimes N}-\kappa_{M}-\kappa_{N}\right)=\gamma_{M, N}, & \\
\Phi\left(t_{s_{i}}\right)=\mathrm{id}_{M} \otimes \mathrm{id}_{N} \otimes \mathrm{id}_{V}^{\otimes(j-1)} \otimes s \otimes \mathrm{id}_{V}^{\otimes(k-j-1)} & \text { for } 1 \leq i \leq k-1,
\end{array}
$$

where $s \cdot(u \otimes v)=v \otimes u$.

Proof. The $t_{s_{i}}$ act by simple transpositions, so they generate an action of $\mathbb{C} S_{k}$ on $V^{\otimes k}$. Since the coproduct is cocommutative, the action of $\mathbb{C} S_{k}$ commutes with the $\mathfrak{g}$-action.

Since $\kappa$ is central, $\kappa_{M, \leq i} \in \operatorname{End}_{\mathfrak{g}}\left(M \otimes V^{\otimes i}\right)$. By (2-13), this means $\kappa_{M, \leq i} \otimes$ $\mathrm{id}_{V}^{j-i}$ is an element of $\operatorname{End}_{\mathfrak{g}}\left(M \otimes V^{\otimes j}\right)$ for $i<j$. Thus the actions of $\kappa_{M, \leq i}$ for $i=1,2, \ldots, k$, and so the actions of $x_{1}, \ldots, x_{k}$, pairwise commute. Similarly, $\left\{y_{1}, \ldots, y_{k}\right\}$ and $\left\{z_{0}, \ldots, z_{k}\right\}$ each act commutatively on $M \otimes N \otimes V^{\otimes k}$. Again by (2-13), these operators are also all contained in $\operatorname{End}_{\mathfrak{g}}\left(M \otimes N \otimes V^{\otimes k}\right)$. Moreover, since $M, N$, and $V$ are simple, $\kappa_{M}, \kappa_{N}$, and $\kappa_{V}$ act as constants. So

$$
\Phi\left(z_{0}+\cdots+z_{i}\right)=\frac{1}{2}\left(\kappa_{M \otimes N, \leq i}+i \kappa_{V}-\kappa_{M}-\kappa_{N}\right)
$$

commutes with $\kappa_{M, \leq j}$ and $\kappa_{N, \leq j}$ for $j \leq i$, verifying (2-8).

The relations in (2-9) follow from

$$
\begin{aligned}
& \Phi\left(t_{s_{i}}\left(x_{i}+x_{i+1}\right)\right)=\frac{1}{2} t_{s_{i}}\left(\kappa_{M, \leq i+1}-\kappa_{M, \leq i-1}\right) \\
& =\frac{1}{2} t_{s_{i}}\left(\gamma_{M, i}+\gamma_{M, i+1}+2 \kappa_{V}+2 \sum_{\ell=1}^{i-1}\left(\gamma_{\ell, i}+\gamma_{\ell, i+1}\right)+2 \gamma_{i, i+1}\right) \quad \text { by }(2-16) \\
& =\frac{1}{2}\left(\gamma_{M, i+1}+\gamma_{M, i}+2 \kappa_{V}+2 \sum_{\ell=1}^{i-1}\left(\gamma_{\ell, i+1}+\gamma_{\ell, i}\right)+2 \gamma_{i+1, i}\right) t_{s_{i}} \\
& =\Phi\left(\left(x_{i}+x_{i+1}\right) t_{s_{i}}\right)
\end{aligned}
$$

(a similar computation confirms $\left.\Phi\left(t_{s_{i}}\left(y_{i}+y_{i+1}\right)\right)=\Phi\left(\left(y_{i}+y_{i+1}\right) t_{s_{i}}\right)\right)$. The action of the symmetric group commutes with the action of $\mathfrak{g}$, and if $j<i, \kappa_{X, \leq j}$ acts by 
the identity on the $i$ and $i+1$ factors of $V^{\otimes k}$. Thus

$$
t_{s_{i}} \kappa_{X, \leq j}=\kappa_{X, \leq j} t_{s_{i}} \quad \text { if } j \neq i \text { and } X=M, N \text {, or } M \otimes N,
$$

is satisfied for all $i \neq j$, which implies (2-7).

Finally, as operators on $M \otimes N \otimes V^{\otimes k}$ via $\Phi$,

$$
x_{i}=\frac{1}{2}\left(\kappa_{M, \leq i}-\kappa_{M, \leq i-1}\right)=\frac{1}{2} \kappa_{V}+\gamma_{M, i}+\sum_{1 \leq \ell<i} \gamma_{\ell, i} \quad \text { by }(2-16)
$$

and similarly

$$
y_{i}=\frac{1}{2} \kappa_{i}+\gamma_{N, i}+\sum_{1 \leq \ell<i} \gamma_{\ell, i} \quad \text { and } \quad z_{i}=\kappa_{V}+\gamma_{N, i}+\gamma_{M, i}+\sum_{1 \leq \ell<i} \gamma_{\ell, i} .
$$

So

$$
m_{i, i+1}=x_{i+1}-t_{s_{i}} x_{i} t_{s_{i}}=y_{i+1}-t_{s_{i}} y_{i} t_{s_{i}}=z_{i+1}-t_{s_{i}} z_{i} t_{s_{i}}=\gamma_{i, i+1} .
$$

So (2-11) and (2-4) are satisfied. Since $t_{s_{i}} t_{s_{i+1}} \gamma_{i, i+1} t_{s_{i+1}} t_{s_{i}}=t_{s_{i}} \gamma_{i, i+2} t_{s_{i}}=\gamma_{i+1, i+2}$, relation (2-10) follows from (2-18).

Remark 2.2. As discussed in the introduction, the degenerate two-boundary braid algebra is meant to be the degeneration of the group algebra of the two-boundary braid group, which is the braid group in a space with two punctures, or "flag poles". The two-boundary braid group is the generalization of the affine braid group used in [Orellana and Ram 2007], and just like the affine braid group, has many centralizer algebras for quantum groups as quotients. Analogously, $\varphi_{k}$ has many familiar centralizer algebras for Lie algebras as quotients, and the map in Theorem 2.1 factors through these quotients (in some cases after applying an automorphism). For example:

(1) When $\mathfrak{g}=\mathfrak{g l}_{n}$ or $\mathfrak{s l}_{n}$ and $V$ is the standard representation, the action of $t_{s_{i}}$ on $V \otimes V$ is the same as that of $\gamma$ on $V \otimes V$. So

(a) when $M$ and $N$ are trivial, the images of $x_{i}$ and $y_{i}$ are linear combinations of the images of $t_{s_{j}}$ for $j=1, \ldots, i-1$, and so the image of $\varphi_{k}$ in $\operatorname{End}\left(M \otimes N \otimes V^{\otimes k}\right)$ is the same as that of the symmetric group $S_{k}$; and

(b) when $M$ is trivial and $N$ is a simple highest weight module, the image of $x_{i}$ is redundant as above, and the image of $\varphi_{k}$ in $\operatorname{End}\left(M \otimes N \otimes V^{\otimes k}\right)$ (after a version of the automorphism in Lemma 4.8 when $\mathfrak{g}=\mathfrak{s l}_{n}$ ) is the same as that of the graded Hecke algebra of type A.

(2) When $\mathfrak{g}=\mathfrak{s o}_{n}$ or $\mathfrak{s p}_{2 n}, V$ is the standard representation, and $M$ is trivial, we hope to see the Brauer algebra in [Brauer 1937] and the degenerate affine Wenzl algebra in [Nazarov 1996] (when $N$ is trivial or not, respectively), in 
which case we expect to see elements that are diagrammatically represented by

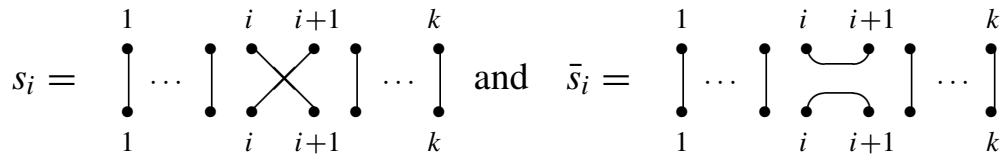

The diagram $s_{i}$ corresponds to $t_{s_{i}}$ in $\varphi_{k}$, and the diagram $\bar{s}_{i}$ corresponds to the element $e_{i}=t_{s_{i}} y_{i}-y_{i+1} t_{s_{i}}-1$. The map $\Phi$ factors through the quotient of $\varphi_{k}$ by the relations in [Nazarov 1996, §4] (after an automorphism in the case where $\mathfrak{g}=\mathfrak{s p}_{2 n}$ ) or by relations in [Daugherty et al. 2011, §2.2] (with no automorphism), as is shown in [Daugherty et al. 2010]. So,

(a) when $M$ and $N$ are trivial, the image of $\varphi_{k}$ in $\operatorname{End}\left(M \otimes N \otimes V^{\otimes k}\right)$ is the same as that of the Brauer algebras, and

(b) when $M$ is trivial and $N$ is a simple highest weight module, the image of $\varphi_{k}$ in $\operatorname{End}\left(M \otimes N \otimes V^{\otimes k}\right)$ is the same as that of the degenerate affine Wenzl algebra.

\section{The degenerate two-boundary Hecke algebra}

Our next goal is to consider the case where $\mathfrak{g}$ is of type $\mathfrak{g l}_{n}$ or $\mathfrak{s l}_{n}$, and $M, N$, and $V$ are three specific $\mathfrak{g}$-modules. In general, even if we specify $V$ to be the standard representation as usual, the decomposition of $M \otimes N$ is not in general multiplicity free, and so the method of considering quotients of the braid algebra in studying centralizers $\operatorname{End}_{\mathfrak{g}}\left(M \otimes N \otimes V^{\otimes k}\right)$ is ineffective. However, in the case where $M$ and $N$ are indexed by rectangular partitions, it is an amazing consequence of the Littlewood-Richardson rule that the decomposition of $M \otimes N$ is multiplicity free. Furthermore, when constructing Hecke algebras in the quantum case, one places quadratic relations on all generators corresponding to the $\mathscr{R}$-matrices. In the degenerate case, these generators are specifically $t_{s_{1}}, \ldots, t_{s_{k-1}}, x_{1}$ and $y_{1}$. By choosing $M$ and $N$ to be indexed by rectangular partitions, we will force quadratic relations on $x_{1}$ and $y_{1}$ as desired.

In this way, we use the representations of $\varphi_{k}$ in Theorem 2.1 to motivate the construction of a new algebra, the degenerate extended two-boundary Hecke algebra. In Section 4 we will carefully lay out the combinatorics behind this construction and explore this motivation further. This section is devoted to the definition and two presentations of $\mathscr{H}_{k}^{\text {ext }}$.

Fix $a, b, p, q \in \mathbb{Z}_{>0}$. The degenerate extended two-boundary Hecke algebra $\mathscr{H}_{k}^{e x t}$ is the quotient of the degenerate two-boundary braid algebra by the relations

$$
t_{s_{i}} x_{i}=x_{i+1} t_{s_{i}}-1 \text { and } t_{s_{i}} y_{i}=y_{i+1} t_{s_{i}}-1 \quad \text { for } i=1, \ldots, k-1 \text {, }
$$


and

$$
\left(x_{1}-a\right)\left(x_{1}+p\right)=0, \quad\left(y_{1}-b\right)\left(y_{1}+q\right)=0 .
$$

The degenerate two-boundary Hecke algebra $\mathscr{H}_{k}$ is the subalgebra of $\mathscr{H}_{k}^{\text {ext }}$ generated by $x_{1}, \ldots, x_{k}, y_{1}, \ldots, y_{k}, z_{1}, \ldots, z_{k}, t_{s_{1}}, \ldots, t_{s_{k-1}}$.

Proposition 3.1 provides a presentation of $\mathcal{H}_{k}^{\text {ext }}$ that is a consolidation of the presentation of $\varphi_{k}$ using the quotient in (3-1) and (3-2). We follow this up with Theorem 3.2, which provides a much more efficient presentation we will make use of in Section 5.

Proposition 3.1. Define

$$
x_{i}=t_{s_{i-1}} x_{i-1} t_{s_{i-1}}+t_{s_{i-1}} \quad \text { and } \quad z_{i}=t_{s_{i-1}} z_{i-1} t_{s_{i-1}}+t_{s_{i-1}} \quad \text { for } i=2, \ldots, k,
$$

and

$m_{1}=0, \quad m_{i}=\sum_{j=1}^{i-1} t_{(j i)} \quad$ for $i>0 \quad$ and $\quad y_{i}=z_{i}-x_{i}+m_{i} \quad$ for $i=1, \ldots, k$.

Then $\mathscr{H}_{k}^{e x t}$ is generated as an algebra over $\mathbb{C}$ by $x_{1}, z_{0}, z_{1}$ and $t_{\mathrm{w}}$ for $\mathrm{w} \in S_{k}$ with relations

$$
t_{\mathrm{w}} t_{\mathrm{w}^{\prime}}=t_{\mathrm{ww}^{\prime}} \quad \text { for } \mathrm{w}, \mathrm{w}^{\prime} \in S_{k}
$$

and the quadratic relations

$$
\left(x_{1}-a\right)\left(x_{1}+p\right)=0, \quad\left(y_{1}-b\right)\left(y_{1}+q\right)=0 \quad \text { for } a, b, p, q \in \mathbb{Z}_{>0},
$$

the commutation relations

$$
\begin{aligned}
& t_{s_{i}} x_{j}=x_{j} t_{s_{i}}, t_{s_{i}} z_{j}=z_{j} t_{s_{i}} \quad \text { for } j \neq i, i+1, \\
& x_{i} x_{j}=x_{j} x_{i}, y_{i} y_{j}=y_{j} y_{i}, z_{i} z_{j}=z_{j} z_{i}, z_{0} z_{i}=z_{i} z_{0} \quad \text { for } 1 \leq i, j \leq k \text {, } \\
& x_{j} z_{i}=z_{i} x_{j} \quad \text { for } i>j \text {, }
\end{aligned}
$$

and the twisting relations

$$
\begin{aligned}
& x_{i}\left(z_{0}+\cdots+z_{i}\right)=\left(z_{0}+\cdots+z_{i}\right) x_{i}, \\
& y_{i}\left(z_{0}+\cdots+z_{i}\right)=\left(z_{0}+\cdots+z_{i}\right) y_{i} \quad \text { for } i=1, \ldots, k .
\end{aligned}
$$

Proof. Equation (2-3) can be rewritten as

$$
\begin{aligned}
m_{j, j+1} & =x_{j+1}-t_{s_{j}} x_{j} t_{s_{j}}=t_{s_{j}}, \\
m_{i, j} & =t_{(i j-1)} m_{j-1, j} t_{(i j-1)}=t_{(i j)} \quad \text { if } i<j-1 .
\end{aligned}
$$


So $m_{1}=0$ and $m_{i}=\sum_{1<j<i} t_{(i j)}$. Therefore (2-4) implies

$$
\begin{aligned}
t_{s_{i}} z_{i} t_{s_{i}} & =t_{s_{i}}\left(x_{i}+y_{i}-m_{i}\right) t_{s_{i}} \\
& =x_{i+1}-t_{s_{i}}+y_{i+1}-t_{s_{i}}-t_{s_{i}}\left(\sum_{1<j<i} t_{(i j)}\right) t_{s_{i}} \\
& =x_{i+i}+y_{i+1}-t_{s_{i}}-t_{s_{i}}-\sum_{1<j<i} t_{(i+1 j)} \\
& =x_{i+i}+y_{i+1}-m_{i}-t_{s_{i}}=z_{i+1}-t_{s_{i}} .
\end{aligned}
$$

Similarly, for $i=1, \ldots, k-1$, any two of

$$
x_{i+1}-t_{s_{i}} x_{i} t_{s_{i}}=t_{s_{i}}, \quad y_{i+1}-t_{s_{i}} y_{i} t_{s_{i}}=t_{s_{i}}, \quad \text { and } \quad z_{i+1}-t_{s_{i}} z_{i} t_{s_{i}}=t_{s_{i}}
$$

imply the third. So we use (3-1) to discard the generators $x_{2}, \ldots, x_{k}, y_{1}, \ldots, y_{k}$, and $z_{2}, \ldots, z_{k}$, by defining

$x_{i}=t_{s_{i-1}} x_{i-1} t_{s_{i-1}}+t_{s_{i-1}} \quad$ and $\quad z_{i}=t_{s_{i-1}} z_{i-1} t_{s_{i-1}}+t_{s_{i-1}} \quad$ for $i=2, \ldots, k$,

and $\quad y_{i}=z_{i}-x_{i}+m_{i} \quad$ for $i=1, \ldots, k$.

Relation (2-9) can be rewritten as

$t_{s_{i}} x_{i}-x_{i+1} t_{s_{i}}=t_{s_{i}}\left(t_{s_{i}} x_{i}-x_{i+1} t_{s_{i}}\right) t_{s_{i}} \quad$ and $\quad t_{s_{i}} y_{i}-y_{i+1} t_{s_{i}}=t_{s_{i}}\left(t_{s_{i}} y_{i}-y_{i+1} t_{s_{i}}\right) t_{s_{i}}$

for $1 \leq i \leq k-1$, which is equivalent to $-1=-1$. Relation (2-10) is equivalent to

$$
\left(t_{s_{i}} t_{s_{i+1}}\right)\left(t_{s_{i}}\right)\left(t_{s_{i+i}} t_{s_{i}}\right)=t_{s_{i+1}} \quad \text { for } 1 \leq i \leq k-2,
$$

which is redundant with relation $t_{s_{i}}^{2}=1$ and the second relation in (2-1). Relation (2-11) is equivalent to $t_{s_{i}}=t_{s_{i}}$. So by introducing (3-1), we can discard relations (2-9)-(2-11). The second relation in (2-7) can also be discarded since

$t_{s_{i}} y_{j}=t_{s_{i}}\left(z_{j}-x_{j}+\sum_{\ell=1}^{j-1} t_{(\ell j)}\right)=\left(z_{j}-x_{j}+\sum_{\ell=1}^{j-1} t_{(\ell j)}\right) t_{s_{i}}=y_{j} t_{s_{i}} \quad$ for $j \neq i, i+1$,

Finally, independent of (3-1), we rewrite relation (2-8) as

$$
\begin{aligned}
& x_{i} z_{0}=z_{0} x_{i}+\left(\left(z_{1}+\cdots+z_{i}\right) x_{i}-x_{i}\left(z_{1}+\cdots+z_{i}\right)\right), \\
& y_{i} z_{0}=z_{0} y_{i}+\left(\left(z_{1}+\cdots+z_{i}\right) y_{i}-y_{i}\left(z_{1}+\cdots+z_{i}\right)\right), \quad \text { for } i=1, \ldots, k,
\end{aligned}
$$

and, for $i>j$,

$$
\begin{aligned}
x_{j} z_{i} & =x_{j}\left(z_{0}+\cdots+z_{i}\right)-x_{j}\left(z_{0}+\cdots+z_{i-1}\right) \\
& =\left(z_{0}+\cdots+z_{i}\right) x_{j}-\left(z_{0}+\cdots+z_{i-1}\right) x_{j}=z_{i} x_{j}, \\
y_{j} z_{i} & =z_{i} y_{j} .
\end{aligned}
$$

The following is a streamlined version of Proposition 3.1, which will be our favorite presentation for calculating representations in Section 5. 
Theorem 3.2. Let

$$
w_{i}=z_{i}-\frac{1}{2}(a-p+b-q) .
$$

$\mathscr{H}_{k}^{\text {ext }}$ is generated as an algebra over $\mathbb{C}$ by $w_{0}, w_{1}, \ldots, w_{k}, x_{1}, t_{s_{1}}, \ldots, t_{s_{k-1}}$ with relations the braid relations

$$
\begin{gathered}
t_{s_{i}}^{2}=1, \quad t_{s_{i}} t_{s_{i+1}} t_{s_{i}}=t_{s_{i+1}} t_{s_{i}} t_{s_{i+1}}, \quad t_{s_{i}} t_{s_{j}}=t_{s_{j}} t_{s_{i}} \quad \text { for }|i-j|>1, \\
x_{1}\left(t_{s_{1}} x_{1} t_{s_{1}}+t_{s_{1}}\right)=\left(t_{s_{1}} x_{1} t_{s_{1}}+t_{s_{1}}\right) x_{1},
\end{gathered}
$$

the quadratic relations

$$
\left(x_{1}-a\right)\left(x_{1}+p\right)=0 \text {, }
$$

the commutation relations

$$
\begin{aligned}
t_{s_{i}} w_{j} & =w_{j} t_{s_{i}} & & \text { for } j \neq i, i+1, \\
x_{1} w_{i} & =w_{i} x_{1} & & \text { for } i=2, \ldots, k, \\
x_{1} t_{s_{i}} & =t_{s_{i}} x_{1} & & \text { for } i=2, \ldots, k-1, \\
w_{i} w_{j} & =w_{j} w_{i} & & \text { for } i, j=0, \ldots, k,
\end{aligned}
$$

and twisting relations

$$
\begin{aligned}
& t_{s_{i}} w_{i}=w_{i+1} t_{s_{i}}-1 \quad \text { for } i=1, \ldots, k-1, \\
& x_{1} w_{0}=w_{0} x_{1}-\left(x_{1} w_{1}-w_{1} x_{1}\right), \\
& x_{1} w_{1}=-w_{1} x_{1}+(a-p) w_{1}+w_{1}^{2}+\frac{1}{4}\left((a+p)^{2}-(b+q)^{2}\right)
\end{aligned}
$$

Proof. With the exception of

$$
y_{i}=z_{i}-x_{i}+m_{i}=w_{i}-x_{i}+m_{i}+\frac{1}{2}(a-p+b-q),
$$

every substitution of $z_{i}=w_{i}+\frac{1}{2}(a-p+b-q)$ in the presentation in Proposition 3.1 results in a cancellation, that is,

$$
\begin{array}{ll}
t_{s_{i}} w_{j}=w_{j} t_{s_{i}} & \text { for } j \neq i, i+1, \\
w_{0} w_{i}=w_{i} w_{0} & \text { for } 1 \leq i, j \leq k, \\
x_{j} w_{i}=w_{i} x_{j} & \text { for } i>j
\end{array}
$$

and

$x_{i}\left(w_{0}+\cdots+w_{i}\right)=\left(w_{0}+\cdots+w_{i}\right) x_{i} \quad$ and $\quad y_{i}\left(w_{0}+\cdots+w_{i}\right)=\left(w_{0}+\cdots+w_{i}\right) y_{i}$, are immediate.

Next, we address (3-12) by proving the following claim: 
Claim 1. The set of relations

$$
\begin{aligned}
& \left(x_{1}-a\right)\left(x_{1}+p\right)=0, \quad\left(y_{1}-b\right)\left(y_{1}+q\right)=0, \\
& w_{1}=x_{1}+y_{1}-\frac{1}{2}(a-p+b-q)
\end{aligned}
$$

are equivalent to the set of relations

$$
\begin{aligned}
& \left(x_{1}-a\right)\left(x_{1}+p\right)=0, \\
& x_{1} w_{1}=-w_{1} x_{1}+(a-p) w_{1}+w_{1}^{2}+\frac{1}{4}\left((a+p)^{2}-(b+q)^{2}\right)
\end{aligned}
$$

Proof. (3-13) implies (3-14): First notice that

$$
\begin{aligned}
& x_{1}^{2}=(a-p) x_{1}+a p, \quad y_{1}^{2}=(b-q) y_{1}+b q, \\
& z_{1}^{2}=\left(x_{1}+y_{1}\right)^{2}=x_{1} y_{1}+y_{1} x_{1}+(a-p) x_{1}+(b-q) y_{1}+a p+b q .
\end{aligned}
$$

So

$$
\begin{aligned}
x_{1} w_{1} & +w_{1} x_{1} \\
& =x_{1}\left(x_{1}+y_{1}-(a-p+b-q) / 2\right)+\left(x_{1}+y_{1}-(a-p+b-q) / 2\right) x_{1} \\
& =2 x_{1}^{2}+\left(x_{1} y_{1}+y_{1} x_{1}\right)-(a-p+b-q) x_{1} \\
& =(a-p-(b-q)) x_{1}+2 a p+\left(x_{1} y_{1}+y_{1} x_{1}\right) .
\end{aligned}
$$

Since

$$
\begin{aligned}
& w_{1}^{2}=z_{1}^{2}-(a-p+b-q) z_{1}+\frac{1}{4}(a-p+b-q)^{2} \\
& =x_{1} y_{1}+y_{1} x_{1}+(a-p) x_{1}+(b-q) y_{1}+a p+b q \\
& -(a-p+b-q)\left(x_{1}+y_{1}\right)+\frac{1}{4}(a-p+b-q)^{2} \\
& =\left(x_{1} y_{1}+y_{1} x_{1}\right)-(b-q) x_{1}-(a-p)\left(w_{1}-x_{1}+(a-p+b-q) / 2\right) \\
& +a p+b q+\frac{1}{4}(a-p+b-q)^{2} \\
& =\left(x_{1} y_{1}+y_{1} x_{1}\right)+(a-p-(b-q)) x_{1}-(a-p) w_{1} \\
& +a p+b q-(a-p)^{2} / 4+(b-q)^{2} / 4
\end{aligned}
$$

we have $x_{1} w_{1}+w_{1} x_{1}$ is equal to

$$
\begin{aligned}
& (a-p-(b-q)) x_{1}+2 a p \\
& +\left(w_{1}^{2}-\left((a-p-(b-q)) x_{1}-(a-p) w_{1}+a p+b q-(a-p)^{2} / 4+(b-q)^{2} / 4\right)\right) \\
& =w_{1}^{2}+(a-p) w_{1}+\frac{1}{4}\left((a+p)^{2}-(b+q)^{2}\right)
\end{aligned}
$$


We show (3-14) implies (3-13): If $y_{1}=w_{1}-x_{1}+\frac{1}{2}(a-p+b-q)$, then using both relations in (3-14) to expand $x_{1}^{2}$ and $w_{1} x_{1}+x_{1} w_{1}$, direct calculation yields

$$
\begin{aligned}
\left(y_{1}-\right. & b)\left(y_{1}+q\right) \\
& =\left(w_{1}-x_{1}+\frac{1}{2}(a-p+b-q)-b\right)\left(w_{1}-x_{1}+\frac{1}{2}(a-p+b-q)+q\right) \\
& =0 .
\end{aligned}
$$

The remainder is showing that the relations in Proposition 3.1 follow from relations (3-3) and (3-4)-(3-12). As in Proposition 3.1, define $x_{i+1}=t_{s_{i}} x_{i} t_{s_{i}}+t_{s_{i}}$. By induction on $\ell$,

$$
x_{i+1}=t_{s_{i}} \cdots t_{s_{\ell+1}}\left(t_{s_{\ell}} x_{\ell} t_{s_{\ell}}+t_{s_{\ell}}\right) t_{s_{\ell+1}} \cdots t_{s_{i}}+\sum_{r=\ell+1}^{i} t_{s_{i}} \cdots t_{s_{r+1}} t_{s_{r}} t_{s_{r+1}} \cdots t_{s_{i}}
$$

Claim 2. $t_{s_{i}} x_{j}=x_{j} t_{s_{i}}$ for $i>j$.

Proof. If $i>j$, then $t_{s_{i}}$ commutes with $t_{s_{\ell}}$ for all $\ell<j$, so by (3-8) and (3-15)

$$
\begin{aligned}
& t_{s_{i}} x_{j}=t_{s_{i}}\left(t_{s_{j-1}} \cdots t_{s_{2}}\right)\left(t_{s_{1}} x_{1} t_{s_{1}}+t_{s_{1}}\right)\left(t_{s_{2}} \cdots t_{s_{j-1}}\right) \\
&+t_{s_{i}} \sum_{\ell=2}^{j-1} t_{s_{j-1}} \cdots t_{s_{\ell+1}} t_{s_{\ell}} t_{s_{\ell+1}} \cdots t_{s_{j-1}} \\
&=\left(t_{s_{j-1}} \cdots t_{s_{2}}\right)\left(t_{s_{1}} x_{1} t_{s_{1}}+t_{s_{1}}\right)\left(t_{s_{2}} \cdots t_{s_{j-1}}\right) t_{s_{i}} \\
&+\left(\sum_{\ell=2}^{j-1} t_{s_{j-1}} \cdots t_{s_{\ell+1}} t_{s_{\ell}} t_{s_{\ell+1}} \cdots t_{s_{j-1}}\right) t_{s_{i}} \\
&=x_{j} t_{s_{i}} .
\end{aligned}
$$

Claim 3. $t_{s_{i}} x_{j}=x_{j} t_{s_{i}}$ for $i<j-1$.

Proof. By (3-15),

$$
\begin{array}{r}
t_{s_{i}} x_{j}=t_{s_{i}}\left(t_{s_{j-1}} \cdots t_{s_{i+2}} t_{s_{i+1}}\right)\left(t_{s_{i}} x_{i} t_{s_{i}}+t_{s_{i}}\right)\left(t_{s_{i+1}} t_{s_{i+2}} \cdots t_{s_{j-1}}\right) \\
+t_{s_{i}} t_{s_{j-1}} \cdots t_{s_{i+2}} t_{s_{i+1}} t_{s_{i+2}} \cdots t_{s_{j-1}} \\
+t_{s_{i}} \sum_{\ell=i+2}^{j-1} t_{s_{j-1}} \cdots t_{s_{\ell+1}} t_{s_{\ell}} t_{s_{\ell+1}} \cdots t_{s_{j-1}} \\
=\left(t_{s_{j-1}} \cdots t_{s_{i+2}}\right)\left(t_{s_{i}}\right)\left(t_{s_{i+1}}\left(t_{s_{i}} x_{i} t_{s_{i}}+t_{s_{i}}\right) t_{s_{i+1}}+t_{s_{i+1}}\right)\left(t_{s_{i+2}} \cdots t_{s_{j-1}}\right) \\
+\left(\sum_{\ell=i+2}^{j-1} t_{s_{j-1}} \cdots t_{s_{\ell+1}} t_{s_{\ell}} t_{s_{\ell+1}} \cdots t_{s_{j-1}}\right) t_{s_{i}}
\end{array}
$$


But, by Claim 2, since $i+1>i$,

$$
\begin{aligned}
t_{s_{i}}\left(t_{s_{i+1}}\left(t_{s_{i}} x_{i} t_{s_{i}}+t_{s_{i}}\right) t_{s_{i+1}}+t_{s_{i+1}}\right) & =t_{s_{i}} t_{s_{i+1}} t_{s_{i}} x_{i} t_{s_{i}} t_{s_{i+1}}+t_{s_{i}} t_{s_{i+1}} t_{s_{i}} t_{s_{i+1}}+t_{s_{i}} t_{s_{i+1}} \\
& =t_{s_{i+1}} t_{s_{i}} t_{s_{i+1}} x_{i} t_{s_{i}} t_{s_{i+1}}+t_{s_{i+1}} t_{s_{i}} t_{s_{i+1}}^{2}+t_{s_{i}} t_{s_{i+1}} t_{s_{i}}^{2} \\
& =t_{s_{i+1}} t_{s_{i}} x_{i} t_{s_{i+1}} t_{s_{i}} t_{s_{i+1}}+t_{s_{i+1}} t_{s_{i}}+t_{s_{i+1}} t_{s_{i}} t_{s_{i+1}} t_{s_{i}} \\
& =t_{s_{i+1}} t_{s_{i}} x_{i} t_{s_{i}} t_{s_{i+1}} t_{s_{i}}+t_{s_{i+1}} t_{s_{i}}+t_{s_{i+1}} t_{s_{i}} t_{s_{i+1}} t_{s_{i}} \\
& =\left(t_{s_{i+1}}\left(t_{s_{i}} x_{i} t_{s_{i}}+t_{s_{i}}\right) t_{s_{i+1}}+t_{s_{i+1}}\right) t_{s_{i}} .
\end{aligned}
$$

So $t_{s_{i}} x_{j}=x_{j} t_{s_{i}}$.

Claim 4. $x_{i} x_{j}=x_{j} x_{i}$ for $i, j=1, \ldots, k$.

Proof. First, $x_{1} x_{2}=x_{2} x_{1}$ by (3-4). Next, we induct on $i$ to show $x_{i} x_{i+1}=x_{i+1} x_{i}$ for $i=1, \ldots, k-1$ :

$$
\begin{aligned}
x_{i} x_{i+1}= & x_{i} t_{s_{i}} x_{i} t_{s_{i}}+x_{i} t_{s_{i}} \\
= & \left(\left(t_{s_{i-1}} x_{i-1} t_{s_{i-1}}+t_{s_{i-1}}\right) t_{s_{i}}\left(t_{s_{i-1}} x_{i-1} t_{s_{i-1}}+t_{s_{i-1}}\right)+\left(t_{s_{i-1}} x_{i-1} t_{s_{i-1}}+t_{s_{i-1}}\right)\right) t_{s_{i}} \\
= & \left(t_{s_{i-1}} x_{i-1} t_{s_{i-1}} t_{s_{i}} t_{s_{i-1}} x_{i-1} t_{s_{i-1}}+t_{s_{i-1}} x_{i-1} t_{s_{i-1}}\right) t_{s_{i}} \\
& \quad \quad+\left(t_{s_{i-1}} t_{s_{i}} t_{s_{i-1}} x_{i-1} t_{s_{i-1}}+t_{s_{i-1}} x_{i-1} t_{s_{i-1}} t_{s_{i}} t_{s_{i-1}}\right) t_{s_{i}}+\left(t_{s_{i-1}} t_{s_{i}} t_{s_{i-1}}+t_{s_{i-1}}\right) t_{s_{i}} .
\end{aligned}
$$

But

$$
\begin{aligned}
\left(t_{s_{i-1}} x_{i-1} t_{s_{i-1}} t_{s_{i}} t_{s_{i-1}} x_{i-1} t_{s_{i-1}}+t_{s_{i-1}} x_{i-1} t_{s_{i-1}}\right) t_{s_{i}} \\
=t_{s_{i-1}} x_{i-1} t_{s_{i}} t_{s_{i-1}} t_{s_{i}} x_{i-1} t_{s_{i-1}} t_{s_{i}}+t_{s_{i-1}} x_{i-1} t_{s_{i}}^{2} t_{s_{i-1}} t_{s_{i}} \\
=t_{s_{i-1}} t_{s_{i}} x_{i-1} t_{s_{i-1}} x_{i-1} t_{s_{i}} t_{s_{i-1}} t_{s_{i}}+t_{s_{i-1}} t_{s_{i}} x_{i-1} t_{s_{i}} t_{s_{i-1}} t_{s_{i}} \\
=t_{s_{i-1}} t_{s_{i}} x_{i-1} t_{s_{i-1}} x_{i-1} t_{s_{i-1}} t_{s_{i}} t_{s_{i-1}}+t_{s_{i-1}} t_{s_{i}} x_{i-1} t_{s_{i-1}} t_{s_{i}} t_{s_{i-1}} \\
=t_{s_{i-1}} t_{s_{i}}\left(x_{i-1} t_{s_{i-1}} x_{i-1} t_{s_{i-1}}+x_{i-1} t_{s_{i-1}}\right) t_{s_{i}} t_{s_{i-1}} \\
=t_{s_{i-1}} t_{s_{i}}\left(t_{s_{i-1}} x_{i-1} t_{s_{i-1}} x_{i-1}+t_{s_{i-1}} x_{i-1}\right) t_{s_{i}} t_{s_{i-1}} \\
=t_{s_{i}}\left(t_{s_{i-1}} x_{i-1} t_{s_{i-1}} t_{s_{i}} t_{s_{i-1}} x_{i-1} t_{s_{i-1}}+t_{s_{i-1}} x_{i-1} t_{s_{i-1}}\right)
\end{aligned}
$$

and

$$
\begin{aligned}
\left(t_{s_{i-1}} t_{s_{i}} t_{s_{i-1}} x_{i-1} t_{s_{i-1}}+t_{s_{i-1}}\right. & \left.x_{i-1} t_{s_{i-1}} t_{s_{i}} t_{s_{i-1}}\right) t_{s_{i}} \\
& =t_{s_{i}} t_{s_{i-1}} x_{i-1} t_{s_{i}} t_{s_{i-1}}+t_{s_{i-1}} x_{i-1} t_{s_{i}} t_{s_{i-1}} \\
& =t_{s_{i}} t_{s_{i-1}} x_{i-1} t_{s_{i}} t_{s_{i-1}}+t_{s_{i}}^{2} t_{s_{i-1}} t_{s_{i}} x_{i-1} t_{s_{i-1}} \\
& =t_{s_{i}}\left(t_{s_{i-1}} x_{i-1} t_{s_{i-1}} t_{s_{i}} t_{s_{i-1}} t_{s_{i-1}} t_{s_{i}} t_{s_{i-1}} x_{i-1} t_{s_{i-1}}\right)
\end{aligned}
$$

and

$$
\left(t_{s_{i-1}} t_{s_{i}} t_{s_{i-1}}+t_{s_{i-1}}\right) t_{s_{i}}=t_{s_{i}} t_{s_{i-1}} t_{s_{i}}^{2}+t_{s_{i}}^{2} t_{s_{i-1}} t_{s_{i}}=t_{s_{i}}\left(t_{s_{i-1}}+t_{s_{i-1}} t_{s_{i}} t_{s_{i-1}}\right) .
$$


So

$$
\begin{aligned}
x_{i} x_{i+1} & =t_{s_{i}}\left(\left(t_{s_{i-1}} x_{i-1} t_{s_{i-1}}+t_{s_{i-1}}\right) t_{s_{i}}\left(t_{s_{i-1}} x_{i-1} t_{s_{i-1}}+t_{s_{i-1}}\right)+\left(t_{s_{i-1}} x_{i-1} t_{s_{i-1}}+t_{s_{i-1}}\right)\right) \\
& =t_{s_{i}} x_{i} t_{s_{i}} x_{i}+t_{s_{i}} x_{i}=x_{i+1} x_{i} .
\end{aligned}
$$

Finally, assume, without loss of generality, that $i<j$. Then Claim 4 follows by (3-15) and Claim 2.

Claim 5. $w_{j} x_{i}=x_{i} w_{j}$ for $j>i$.

Proof. By (3-15),

$$
x_{i}=t_{s_{i-1}} \cdots t_{s_{1}} x_{1} t_{s_{1}} \cdots t_{s_{i-1}}+\sum_{\ell=1}^{i-1} t_{s_{i-1}} \cdots t_{s_{\ell+1}} t_{s_{\ell}} t_{s_{\ell+1}} \cdots t_{s_{i-1}} .
$$

So (3-6) and (3-7) imply $w_{j} x_{i}=x_{i} w_{j}$ for $j>i$.

Claim 6. $x_{i}\left(w_{0}+\cdots+w_{i}\right)=\left(w_{0}+\cdots+w_{i}\right) x_{i}$ for $i=1, \ldots k$.

Proof. This follows by induction on $i$, with $i=1$ satisfied by (3-11). Rewrite (3-10) as $t_{s_{i}}\left(w_{i}+w_{i+1}\right)=\left(w_{i}+w_{i+1}\right) t_{s_{i}}$, so

$$
\begin{aligned}
x_{i}\left(w_{0}+\cdots+w_{i}\right) & =\left(t_{s_{i-1}} x_{i-1} t_{s_{i-1}}+t_{s_{i-1}}\right)\left(w_{0}+\cdots+w_{i}\right) \\
& =\left(w_{0}+\cdots+w_{i}\right)\left(t_{s_{i-1}} x_{i-1} t_{s_{i-1}}+t_{s_{i-1}}\right)=\left(w_{0}+\cdots+w_{i}\right) x_{i}
\end{aligned}
$$

since $x_{i-1} w_{i}=w_{i} x_{i-1}$ by Claim 5, and $t_{s_{\ell}} w_{j}=w_{j} t_{s_{\ell}}$ for $\ell<j$ by (3-6).

Claim 7. If $y_{1}=w_{1}-x_{1}+\frac{1}{2}(a-p+b-q)$ and $y_{2}=w_{2}-x_{2}+t_{s_{1}}+\frac{1}{2}(a-p+b-q)$, then $y_{1} y_{2}=y_{2} y_{1}$ and $y_{1} t_{s_{i}}=t_{s_{i}} y_{1}$ for $i>1$.

Proof. Let $K=\frac{1}{2}(a-p+b-q)$. So

$$
\begin{aligned}
y_{1} y_{2} & =\left(w_{1}-x_{1}+K\right)\left(w_{2}-\left(t_{s_{1}} x_{1} t_{s_{1}}+t_{s_{1}}\right)+t_{s_{1}}+K\right) \\
& =\left(w_{2}+K\right)\left(w_{1}-x_{1}+K\right)-\left(w_{1}-x_{1}+K\right) t_{s_{1}} x_{1} t_{s_{1}} \\
& =\left(w_{2}+K\right)\left(w_{1}-x_{1}+K\right)-\left(t_{s_{1}} x_{1} t_{s_{1}}\right) K-w_{1} t_{s_{1}} x_{1} t_{s_{1}}+x_{1} t_{s_{1}} x_{1} t_{s_{1}} \\
& =\left(w_{2}+K\right)\left(w_{1}-x_{1}+K\right)-\left(t_{s_{1}} x_{1} t_{s_{1}}\right) K+x_{1} t_{s_{1}} x_{1} t_{s_{1}}-\left(t_{s_{1}} w_{2}-1\right) x_{1} t_{s_{1}} \\
& =\left(w_{2}+K\right)\left(w_{1}-x_{1}+K\right)-\left(t_{s_{1}} x_{1} t_{s_{1}}\right) K+x_{1} t_{s_{1}} x_{1} t_{s_{1}}+x_{1} t_{s_{1}}-t_{s_{1}} x_{1} w_{2} t_{s_{1}} \\
& =\left(w_{2}+K\right)\left(w_{1}-x_{1}+K\right)-\left(t_{s_{1}} x_{1} t_{s_{1}}\right) K+t_{s_{1}} x_{1} t_{s_{1}} x_{1}+t_{s_{1}} x_{1}-t_{s_{1}} x_{1}\left(t_{s_{1}} w_{1}+1\right) \\
& =\left(w_{2}+K\right)\left(w_{1}-x_{1}+K\right)-\left(t_{s_{1}} x_{1} t_{s_{1}}\right) K+t_{s_{1}} x_{1} t_{s_{1}} x_{1}-t_{s_{1}} x_{1} t_{s_{1}} w_{1} \\
& =\left(w_{2}-t_{s_{1}} x_{1} t_{s_{1}}+K\right)\left(w_{1}-x_{1}+K\right) \\
& =y_{2} y_{1} .
\end{aligned}
$$

The latter is simply $t_{s_{i}}$ commuting with $w_{1}, x_{1}$, and $K$ for $i>1$. 
Claim 8. Let $m_{i}=\sum_{j=1}^{i-1} t_{(j i)}$ and $K=\frac{1}{2}(a-p+b-q)$. If $y_{1}=w_{1}-x_{1}+K$, then

$$
y_{i}=w_{i}-x_{i}+m_{i}+K \quad \text { and } \quad y_{i}=t_{s_{i-1}} y_{i-1} t_{s_{i-1}}+t_{s_{i-1}}
$$

for $i=2, \ldots, k$ are equivalent definitions of $y_{i}$.

Proof. Since $t_{s_{j}} t_{(i j)} t_{s_{j}}=t_{(i j+1)}$, we have $t_{s_{j}} m_{j} t_{s_{j}}=m_{j+1}-t_{s_{j}}$, and so

$$
\begin{aligned}
t_{s_{i}} y_{i} t_{s_{i}}+t_{s_{i}} & =t_{s_{i}}\left(w_{i}-x_{i}+m_{i}+K\right) t_{s_{i}}+t_{s_{i}} \\
& =\left(w_{i+1}-t_{s_{i}}\right)-\left(x_{i+1}-t_{s_{i}}\right)+\left(m_{i+1}-t_{s_{i}}\right)+K+t_{s_{i}} \\
& =w_{i+1}-x_{i+1}+m_{i+1}+K=y_{i+1} .
\end{aligned}
$$

The other direction follows by induction.

Claim 9. If $y_{i}$ is as in Claim 8, then

$$
\begin{aligned}
& y_{i} y_{j}=y_{j} y_{i} \quad \text { for } i, j=1, \ldots, k, \quad t_{s_{i}} y_{j}=y_{j} t_{s_{i}} \quad \text { for } j \neq i, i+1, \\
& y_{i} w_{0}=w_{0} y_{i}+\left(\left(w_{1}+\cdots+w_{i}\right) y_{i}-y_{i}\left(w_{1}+\cdots+w_{i}\right)\right) \quad \text { for } i=1, \ldots k .
\end{aligned}
$$

Proof. These follow from Claims 7 and 8 analogously to the $x_{i}$-valued relations above.

This completes the proof of Theorem 3.2.

As a final remark, Theorem 3.2 implies $\mathscr{H}_{k}^{\text {ext }} \cong \mathbb{C}\left[w_{0}\right] \otimes \mathscr{H}_{k}$ as vector spaces.

\section{Tensor space as a $\mathscr{H}_{k}^{\text {ext }}$-module}

Now we fix $\mathfrak{g}=\mathfrak{g l}_{n}$ or $\mathfrak{s l}_{n}$, and show that for special choices of $\mathfrak{g}$-modules $M, N$, and $V$, the algebra $\mathcal{H}_{k}^{e x t}$ acts on tensor space $M \otimes N \otimes V^{\otimes k}$ by a twist of the representation $\Phi$ in Theorem 2.1 via an automorphism of $\mathscr{G}_{k}$. We go on to explore seminormal representations arising from this representation.

4.1. Preliminaries on $\mathfrak{g l}_{\boldsymbol{n}}$ and $\mathfrak{s l}_{n}$. Let $V=\mathbb{C}^{n}$ with orthonormal basis $\left\{v_{1}, \ldots, v_{n}\right\}$. We consider the Lie algebras

$$
\mathfrak{g l}_{n}=\operatorname{End}(V) \quad \text { and } \quad \mathfrak{s l}_{n}=\{x \in \operatorname{End}(V) \mid \operatorname{tr}(x)=0\} .
$$

Let $\varepsilon_{1}, \ldots, \varepsilon_{n}$ be the orthonormal basis of the weight space $\mathfrak{h}^{*}$, where if $E_{i, j}$ in $\operatorname{End}(V)$ is given by $E_{i, j} v_{k}=\delta_{j, k} v_{i}$, then $\varepsilon_{i}\left(E_{j, j}\right)=\delta_{i, j}$. The set of positive roots is given by

$$
R^{+}=\left\{\varepsilon_{i}-\varepsilon_{j} \mid 1 \leq i<j \leq n\right\} .
$$

The set of roots is $R=R^{+} \cup R^{-}$, where $R^{-}=\left\{-\alpha \mid \alpha \in R^{+}\right\}$, and has basis $\left\{\alpha_{i}=\varepsilon_{i}-\varepsilon_{i+1} \mid i=1, \ldots, n-1\right\}$. 


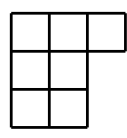

$\mathfrak{g}=\mathfrak{s l}_{n}$

(I)

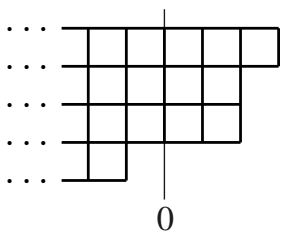

$\mathfrak{g}=\mathfrak{g l}_{n}$

(II)

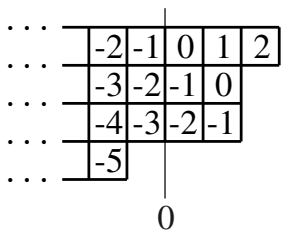

(III)

Figure 1. Weights represented as multisegments. (I) Partition associated to $\lambda=3 \varepsilon_{1}+2 \varepsilon_{2}+2 \varepsilon_{3}-\frac{7}{n}\left(\varepsilon_{1}+\cdots+\varepsilon_{n}\right)$. (II) Multisegment associated to $\lambda=3 \varepsilon_{1}+2 \varepsilon_{2}+2 \varepsilon_{3}-\varepsilon_{4}$. (III) Multisegment from (II) filled in with contents, as defined in (4-4).

The finite-dimensional irreducible $\mathfrak{s l}_{n}$-modules are indexed by the dominant integral weights

$$
P^{+}=\left\{\begin{array}{l|l}
\lambda=\lambda_{1} \varepsilon_{1}+\cdots+\lambda_{n-1} \varepsilon_{n-1}-\frac{|\lambda|}{n}\left(\varepsilon_{1}+\cdots+\varepsilon_{n}\right) & \begin{array}{l}
\lambda_{i} \in \mathbb{Z}_{\geq 0}, \\
\lambda_{1} \geq \cdots \geq \lambda_{n-1} \geq 0, \\
|\lambda|=\lambda_{1}+\cdots+\lambda_{n-1}
\end{array}
\end{array}\right\},
$$

and we identify each weight $\lambda$ with the partition with $\lambda_{i}$ boxes in row $i$. The finite-dimensional irreducible $\mathfrak{g l}_{n}$-modules are indexed by the dominant integral weights

$$
P^{+}=\left\{\lambda=\lambda_{1} \varepsilon_{1}+\cdots+\lambda_{n} \varepsilon_{n} \mid \lambda_{i} \in \mathbb{Z}, \lambda_{1} \geq \cdots \geq \lambda_{n}\right\},
$$

and we identify each weight $\lambda$ the partition which extends infinitely to the left, and ends on the right in column $\lambda_{i}$. For examples of each, see Figure 1 parts (I) and (II). In the case where $\mathfrak{g}=\mathfrak{g l}_{n}$ and $\lambda_{i} \geq 0$ for all $1 \leq i \leq n$, we often represent $\lambda$ as a finite partition, leaving off boxes to the left of 0 . In either case, the first fundamental weight is indexed by a single box, that is, it is given by

$$
\omega_{1}= \begin{cases}\varepsilon_{1} & \mathfrak{g}=\mathfrak{g l}_{n}, \\ \varepsilon_{1}-\frac{1}{n}\left(\varepsilon_{1}+\cdots+\varepsilon_{n}\right) & \mathfrak{g}=\mathfrak{s l}_{n} .\end{cases}
$$

Now let $L(\lambda)$ be the finite-dimensional irreducible highest weight $\mathfrak{g}$-module of weight $\lambda$, that is, the irreducible $\mathfrak{g}$-module generated by highest weight vector $v_{\lambda}^{+}$ of weight $\lambda$ with action

$$
h v_{\lambda}^{+}=\lambda(h) v_{\lambda}^{+} \quad \text { and } \quad x v_{\lambda}^{+}=0 \quad \text { for } h \in \mathfrak{h}, x \in \mathfrak{n}^{+} .
$$

In particular, when $\mathfrak{g}=\mathfrak{g l}_{n}$ or $\mathfrak{s l}_{n}$, the standard representation is $L\left(\omega_{1}\right)$. 
We can calculate the decomposition numbers for the tensor product of two highest weight modules using the Littlewood-Richardson rule [Macdonald 1995, I,1]. The two special cases we require are as follows.

Example 4.1 (adding a box). For $\mathfrak{g}=\mathfrak{g l}_{n}$ or $\mathfrak{s l}_{n+1}$ and $\mu \in P^{+}$,

$$
L(\mu) \otimes L\left(\omega_{1}\right)=\bigoplus_{\lambda \in \mu^{+}} L(\lambda), \quad \text { where } \mu^{+}=\left\{\begin{array}{l}
\text { partitions of height } \leq n \\
\text { obtained by adding a box to } \mu
\end{array}\right\} .
$$

Example 4.2 (rectangles [Stanley 1986, Lemma 3.3; Okada 1998, Theorem 2.4]). Let $p \geq q$ and $a, b$ be nonnegative integers. Denote the rectangular partition with $p$ rows of length $a$ by $\left(a^{p}\right)$. Then each $L(\lambda)$ has multiplicity 1 in $L\left(\left(a^{p}\right)\right) \otimes L\left(\left(b^{q}\right)\right)$ if $\lambda \in \mathscr{P}$, and is zero otherwise, where $\mathscr{P}=\mathscr{P}\left(\left(a^{p}\right),\left(b^{q}\right)\right)$ is the set of partitions $\lambda$ with height $\leq p+q$ such that

$$
\begin{aligned}
& \lambda_{q+1}=\lambda_{q+2}=\cdots=\lambda_{p}=a, \\
& \lambda_{q} \geq \max (a, b), \\
& \lambda_{i}+\lambda_{p+q-i+1}=a+b \quad \text { for } i=1, \ldots, q .
\end{aligned}
$$

In other words, $\mathscr{P}$ is the set of partitions made by placing $\left(b^{q}\right)$ to the right of $\left(a^{p}\right)$, carving a corner out of $\left(b^{q}\right)$, rotating it $180^{\circ}$ and gluing it to the bottom of $\left(a^{p}\right)$. For example,
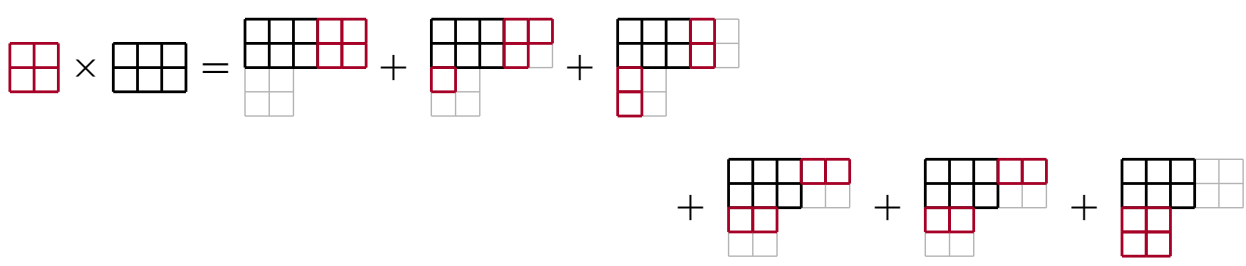

A useful visualization of these partitions is given in Figure 2.

4.1.1. The Casimir element and the operator $\gamma$. When $\mathfrak{g}=\mathfrak{s l}_{n}$, we distinguish the weight

$$
\rho=\frac{1}{2} \sum_{\alpha \in R^{+}} \alpha=\frac{1}{2} \sum_{i=1}^{n}(n+1-2 i) \varepsilon_{i} .
$$

When $\mathfrak{g}=\mathfrak{g l}_{n}$, we choose the analogous weight

$$
\delta=(n-1) \varepsilon_{1}+(n-2) \varepsilon_{2}+\cdots+\varepsilon_{n-1}=\sum_{i=1}^{n}(n-i) \varepsilon_{i},
$$

which matches [Macdonald 1995, I,1]. Keeping $\mathfrak{g}=\mathfrak{g l}_{n}$ or $\mathfrak{s l}_{n}$, recall from (2-14) and (2-15) that

$$
\kappa=\sum_{i} b_{i} b_{i}^{*} \quad \text { and } \quad \gamma=\sum_{i} b_{i} \otimes b_{i}^{*}
$$



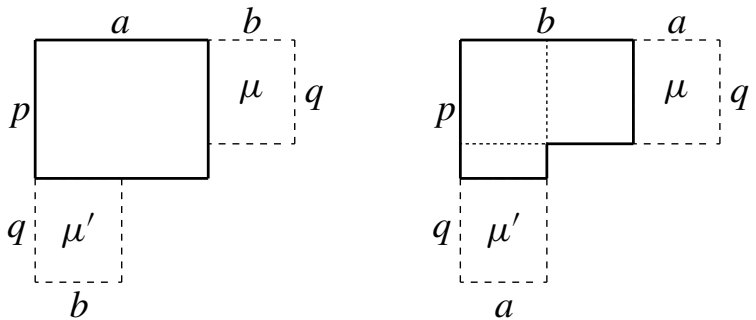

Figure 2. An illustration of partitions in $\mathscr{P}=\mathscr{P}\left(\left(a^{p}\right),\left(b^{q}\right)\right)$. Outlined sections are filled full with boxes, and dashed regions are filled with complementary partitions. At left, the case $a>b: \mu$ is a partition in a $b \times q$ box, and $\mu^{\prime}$ is the $180^{\circ}$ rotation of $\left(b^{q}\right) / \mu$. At right, the case $a<b: \mu$ is a partition in an $a \times q$ box, and $\mu^{\prime}$ is the $180^{\circ}$ rotation of $\left(a^{q}\right) / \mu$.

Lemma 4.1. The Casimir element $\kappa$ acts on $L(\lambda)$ by the constant

$$
\kappa_{L(\lambda)}= \begin{cases}\langle\lambda, \lambda+2 \delta\rangle-(n-1)|\lambda| & \text { when } \mathfrak{g}=\mathfrak{g l}_{n}, \\ \langle\lambda, \lambda+2 \rho\rangle & \text { when } \mathfrak{g}=\mathfrak{s l}_{n} .\end{cases}
$$

It follows that if $L(\lambda)$ is a submodule of $L(\mu) \otimes L(\nu)$, then $\gamma$ acts on the $L(\lambda)$ isotypic component of $L(\mu) \otimes L(v)$ by the constant

$$
\gamma_{\mu \nu}^{\lambda}= \begin{cases}\frac{1}{2}(\langle\lambda, \lambda+2 \delta\rangle-\langle\mu, \mu+2 \delta\rangle-\langle\nu, v+2 \delta\rangle) & \text { when } \mathfrak{g}=\mathfrak{g l}_{n}, \\ \frac{1}{2}(\langle\lambda, \lambda+2 \rho\rangle-\langle\mu, \mu+2 \rho\rangle-\langle\nu, v+2 \rho\rangle) & \text { when } \mathfrak{g}=\mathfrak{s l}_{n} .\end{cases}
$$

Proof. Both cases are classical results. We include here an argument for $\mathfrak{g}=\mathfrak{g l}_{n}$, as it is illustrative of both. For the action of $\kappa$ when $\mathfrak{g}=\mathfrak{s l}_{n}$, see also [Jacobson 1962, Section 8.2]. The elementary matrices $\left\{E_{i j} \mid 1 \leq i, j \leq n\right\}$ form a basis of $\mathfrak{g l}_{n}$ with dual basis $\left\{E_{j i} \mid 1 \leq i, j \leq n\right\}$ with respect to the trace form. So

$$
\kappa=\sum_{1 \leq i, j \leq n} E_{i j} E_{j i}=\sum_{i=1}^{n} E_{i i} E_{i i}+\sum_{1 \leq i<j \leq n}\left(E_{i i}-E_{j j}+2 E_{j i} E_{i j}\right),
$$

and therefore

$$
\begin{aligned}
\kappa v_{\lambda}^{+} & =\left(\sum_{i=1}^{n} \lambda_{i}^{2}+\sum_{1 \leq i<j \leq n} \lambda_{i}-\lambda_{j}+0\right) v_{\lambda}^{+} \\
& =\left(\langle\lambda, \lambda\rangle+\sum_{i=1}^{n}((n-i)-(i-1)) \lambda_{i}\right) v_{\lambda}^{+} \\
& =\left(\langle\lambda, \lambda\rangle+\sum_{i=1}^{n}(2 n-2 i) \lambda_{i}-(n-1) \lambda_{i}\right) v_{\lambda}^{+}=(\langle\lambda, \lambda\rangle+\langle\lambda, 2 \delta\rangle-(n-1)|\lambda|) v_{\lambda}^{+} .
\end{aligned}
$$


Since $\kappa$ acts on $L(\mu) \otimes L(\nu)$ by $\left(\kappa \otimes \mathrm{id}_{L(\nu)}\right)+\left(\mathrm{id}_{L(\mu)} \otimes \kappa\right)+2 \gamma$,

$$
\gamma_{\mu \nu}^{\lambda}=\frac{1}{2}(\langle\lambda, \lambda+2 \delta\rangle-\langle\mu, \mu+2 \delta\rangle-\langle\nu, \nu+2 \delta\rangle)-\frac{1}{2}(n-1)(|\lambda|-|\mu|-|\nu|) .
$$

If $L(\lambda) \subseteq L(\mu) \otimes L(\nu)$, then $|\lambda|=|\mu|+|\nu|$, so the desired action of $\gamma$ follows.

If $B$ is the box in column $c$ and row $r$ of a partition $\lambda$, the content of $B$ is

$$
c(B)=c-r .
$$

See Figure 1 part (III) for an example of a filling of boxes in a multisegment with their respective contents. We can now give a combinatorial description of the values $\gamma$ takes on tensor products in the special cases described in Examples 4.1 and 4.2.

Lemma 4.2. If $L(\lambda)$ is a submodule of $L(\mu) \otimes L\left(\omega_{1}\right)$, then $\gamma$ acts on the $L(\lambda)$ isotypic component of $L(\mu) \otimes L\left(\omega_{1}\right)$ by the constant

$$
\gamma_{\mu \omega_{1}}^{\lambda}= \begin{cases}c(\lambda / \mu) & \text { if } \mathfrak{g}=\mathfrak{g l}_{n}, \\ c(\lambda / \mu)-|\mu| / n & \text { if } \mathfrak{g}=\mathfrak{s l}_{n},\end{cases}
$$

where $\lambda / \mu$ is the box added to $\mu$ to obtain $\lambda$.

Proof. These values are also known in the literature, but we give an illustrative calculation. Let $\mathfrak{g}=\mathfrak{g l}_{n}$ and write $\mu=\mu_{1} \varepsilon_{1}+\cdots+\mu_{n} \varepsilon_{n}$. Adding a box to $\mu$ in the $i$-th row is equivalent to adding $\varepsilon_{i}$ to $\mu$. So, since $\omega_{1}=\varepsilon_{1}$, by Lemma 4.1,

$$
\begin{aligned}
2 \gamma_{\mu \omega_{1}}^{\lambda} & =\left(\left\langle\mu+\varepsilon_{i}, \mu+\varepsilon_{i}+2 \delta\right\rangle-\langle\mu, \mu+2 \delta\rangle-\left\langle\omega_{1}, \omega_{1}+2 \delta\right\rangle\right) \\
& =2\left\langle\mu, \varepsilon_{1}\right\rangle+2\left\langle\varepsilon_{i}-\varepsilon_{1}, \mu\right\rangle+2\left\langle\varepsilon_{i}-\varepsilon_{1}, \varepsilon_{1}\right\rangle+\left\langle\varepsilon_{i}-\varepsilon_{1}, \varepsilon_{i}-\varepsilon_{1}+2 \delta\right\rangle \\
& =2\left(\mu_{1}+\mu_{i}-\mu_{1}-1+1+(n-i)-(n-1)\right)=2\left(\mu_{i}+1-i\right) .
\end{aligned}
$$

A box added to row $i$ of $\mu$ is in position $\left(i, \mu_{i}+1\right)$ and has content $\left(\mu_{i}+1\right)-i$, so $\gamma_{\mu \omega_{1}}^{\lambda}=c(\lambda / \mu)$.

The case where $\mathfrak{g}=\mathfrak{s l}_{n}$ follows analogously, since adding a box to $\mu$ in the $i$-th is equivalent to adding $\varepsilon_{i}-\left(\varepsilon_{1}+\cdots+\varepsilon_{n}\right) / n$ to $\mu$, so $\lambda=\mu+\varepsilon_{i}-\varepsilon_{1}+\omega_{1}$.

Finally, we will need to understand the action of $\gamma$ on $M \otimes N$, where $M$ and $N$ are indexed by rectangular partitions. Specifically, fix $a, b, p, q$ positive integers with $p \geq q$ and

$$
p+q \leq \begin{cases}n & \text { if } \mathfrak{g}=\mathfrak{g l}_{n}, \\ n-1 & \text { if } \mathfrak{g}=\mathfrak{s l}_{n} .\end{cases}
$$

Let $M=L\left(\left(a^{p}\right)\right)$ and $N=L\left(\left(b^{q}\right)\right)$, and recall from Example 4.2 that nontrivial submodules of $M \otimes N$ have multiplicity 1 and are indexed by partitions in $\mathscr{P}$.

Remark 4.3. As a consequence of the description of $\mathscr{P}$ in (4-1), if a box in $\lambda \in$ $\mathscr{P}\left(\left(a^{b}\right),\left(p^{q}\right)\right)$ is moved from position $(i, j)$ to form another partition in $\mathscr{P}$, it must be moved to position $(a+b+1-i, p+q+1-j)$. 
Any partition in $\mathscr{P}$ can be built iteratively by beginning with the partition

$$
\left(a^{p}\right)+\left(b^{q}\right)=\left\{\begin{array}{lr}
a\left(\varepsilon_{1}+\cdots+\varepsilon_{p}\right)+b\left(\varepsilon_{1}+\cdots+\varepsilon_{q}\right) & \text { when } \mathfrak{g}=\mathfrak{g l}_{n}, \\
a\left(\varepsilon_{1}+\cdots+\varepsilon_{p}\right)+b\left(\varepsilon_{1}+\cdots+\varepsilon_{q}\right)-\frac{a p+b q}{n}\left(\varepsilon_{1}+\cdots+\varepsilon_{n}\right) & \text { when } \mathfrak{g}=\mathfrak{s l}_{n}
\end{array}\right.
$$

and moving successive boxes down. Figure 3 gives an example of this process.

Lemma 4.4. Let $\mu$ and $\lambda$ index distinct nontrivial components of $M \otimes N$, assume $\lambda$ differs from $\mu$ by moving one box from position $\left(\mu_{i}, i\right)$. Denote the constant by which $\kappa$ acts on an irreducible component $L(v)$ as $\kappa_{L(v)}$. Then

$$
\kappa_{L(\lambda)}=\kappa_{L(\mu)}-4\left(\left(\mu_{i}-i\right)-\frac{1}{2}(a-p+b-q)\right) .
$$

Proof. If $\mathfrak{g}=\mathfrak{g l}_{n}$ and $\lambda=\mu-\varepsilon_{i}+\varepsilon_{j}$ is obtained from $\mu$ by moving a box from row $i$ into row $j$, then

$$
\begin{aligned}
\kappa_{L(\lambda)} & =\langle\lambda, \lambda+2 \delta\rangle-(n-1)|\lambda| \\
& =\langle\mu, \mu+2 \delta\rangle-(n-1)|\mu|+2\left\langle\mu, \varepsilon_{j}-\varepsilon_{i}\right\rangle+\left\langle\varepsilon_{j}-\varepsilon_{i}, \varepsilon_{j}-\varepsilon_{i}+2 \delta\right\rangle \\
& =\kappa_{L(\mu)}-2\left(\left(\mu_{i}-i\right)-\left(\lambda_{j}-j\right)\right) \\
& \left.=\kappa_{L(\mu)}-2 \text { (content of old box }- \text { content of new box }\right) .
\end{aligned}
$$

A similar computation will show the same for $\mathfrak{g}=\mathfrak{s l}_{n}$. Now, if $\lambda$ and $\mu$ are both elements of $\mathscr{P}$, then $j=p+q+1-i$ and $\lambda_{j}=a+b+1-\mu_{i}$. So

$$
\begin{aligned}
\kappa_{L(\lambda)} & =\kappa_{L(\mu)}-2\left(\left(\mu_{i}-i\right)-\left((a-p)+(b-q)-\left(\mu_{i}-i\right)\right)\right) \\
& =\kappa_{L(\mu)}-4\left(\left(\mu_{i}-i\right)-\frac{1}{2}(a-p+b-q)\right) .
\end{aligned}
$$

Lemma 4.5. Let $\lambda \in \mathscr{P}$ and define $\mathscr{B}_{\lambda}$ to be the set of boxes in $\lambda$ in rows $p+1$ and below. Then $\gamma$ acts on an irreducible component $L(\lambda)$ of $L\left(\left(a^{p}\right)\right) \otimes L\left(\left(b^{q}\right)\right)$ by the constant

$$
\gamma_{\left(a^{p}\right)\left(b^{q}\right)}^{\lambda}= \begin{cases}a b q+2 \sum_{B \in \mathscr{B}_{\lambda}}\left(c(B)-\frac{1}{2}(a-p+b-q)\right) & \text { if } \mathfrak{g}=\mathfrak{g l}_{n}, \\ a b q-a b p q / n+2 \sum_{B \in \mathscr{B}_{\lambda}}\left(c(B)-\frac{1}{2}(a-p+b-q)\right) & \text { if } \mathfrak{g}=\mathfrak{s l}_{n} .\end{cases}
$$

Proof. Both cases proceed similarly by a direct calculation.

Let $\mathfrak{g}=\mathfrak{g l}_{n}$. By Lemma 4.1,

$$
\begin{aligned}
\kappa_{L\left(\left(a^{p}\right)+\left(b^{q}\right)\right)=} & \left\langle\left(a^{p}\right)+\left(b^{q}\right),\left(a^{p}\right)+\left(b^{q}\right)+2 \delta\right\rangle-(n-1)(a p+b q) \\
= & \left\langle\left(a^{p}\right),\left(a^{p}\right)+2 \delta\right\rangle-(n-1) a p \\
& \quad+\left\langle\left(b^{q}\right),\left(b^{q}\right)+2 \delta\right\rangle-(n-1) b q+2\left\langle\left(a^{p}\right),\left(b^{q}\right)\right\rangle \\
= & \kappa_{M}+\kappa_{N}+2\left\langle a\left(\varepsilon_{1}+\cdots+\varepsilon_{p}\right), b\left(\varepsilon_{1}+\cdots+\varepsilon_{q}\right)\right\rangle \\
= & \kappa_{M}+\kappa_{N}+2 a b q .
\end{aligned}
$$


Since any partition indexing a component of $M \otimes N$ can be arrived at recursively by beginning with $\lambda_{0}=\left(a^{p}\right)+\left(b^{q}\right)$ and moving boxes down, iteratively applying Lemma 4.4 implies

$$
\begin{aligned}
\kappa_{L(\lambda)} & =\kappa_{L\left(\lambda_{0}\right)}+4 \sum_{B \in \mathscr{B}_{\lambda}}\left(c(B)-\frac{1}{2}(a-p+b-q)\right) \\
& =\kappa_{M}+\kappa_{N}+2 a b q+4 \sum_{B \in \mathscr{B}_{\lambda}}\left(c(B)-\frac{1}{2}(a-p+b-q)\right) .
\end{aligned}
$$

So $\gamma$ acts on the $L(\lambda)$ component of $M \otimes N$ by

$$
\gamma_{\left(a^{p}\right),\left(b^{q}\right)}^{\lambda}=a b q+2 \sum_{B \in \mathscr{B}_{\lambda}}\left(c(B)-\frac{1}{2}(a-p+b-q)\right) .
$$

In the case where $\mathfrak{g}=\mathfrak{s l}_{n}$,

$$
\kappa_{L\left(\left(a^{p}\right)+\left(b^{q}\right)\right)}=\kappa_{M}+\kappa_{N}+2 a b q-2 a p b q / n,
$$

and so the desired result follows analogously.

An illustration of Lemmas 4.4 and 4.5 is given in Figure 3. We now have all of the machinery needed to rework the representation of the braid algebra $\varphi_{k}$ from Section 2.1 into a representation of $\mathscr{H}_{k}^{\text {ext }}$, which we will do in Section 4.2. However, the recursive process in Figure 3 suggests something further about those partitions obtained by adding a box to a partition in $\mathscr{P}$, as we explore in the following two lemmas. These results will prove useful later in Section 5. Let $\mathscr{P}_{1}$ be the set of partitions that are obtained by adding a box to an element of $\mathscr{P}$.

Lemma 4.6. If $\mu \in \mathscr{P}_{1}\left(\left(a^{p}\right),\left(b^{q}\right)\right)$, then there are exactly one or two $\lambda \in \mathscr{P}$ for which $\lambda \subseteq \mu$.

Proof. As described in Example 4.2, $\mathscr{P}$ is the set of partitions $\lambda$ with height $\leq p+q$ such that

$$
\begin{aligned}
& \lambda_{q+1}=\lambda_{q+2}=\cdots=\lambda_{p}=a, \quad \lambda_{q} \geq \max (a, b), \\
& \lambda_{i}+\lambda_{p+q-i+1}=a+b \quad \text { for } i=1, \ldots, q .
\end{aligned}
$$

Again, a useful visualization of these partitions is provided in Figure 2. As stated in Remark 4.3, if a box is removed from $\lambda \in \mathscr{P}$ in position $(i, j)$, then a box must be added to position $(a+b+1-i, p+q+1-j)$ to get another partition in $\mathscr{P}$. Consider a partition $\mu \in \mathscr{P}_{1}\left(\left(a^{p}\right),\left(b^{q}\right)\right)$. Assume, in addition to having $p \geq q$, that if $p=q$ then $a \geq b$. By moving through the criteria in (4-5) and considering addable boxes for a partition that meets these criteria, we can see that this partition falls into one of the following categories. 


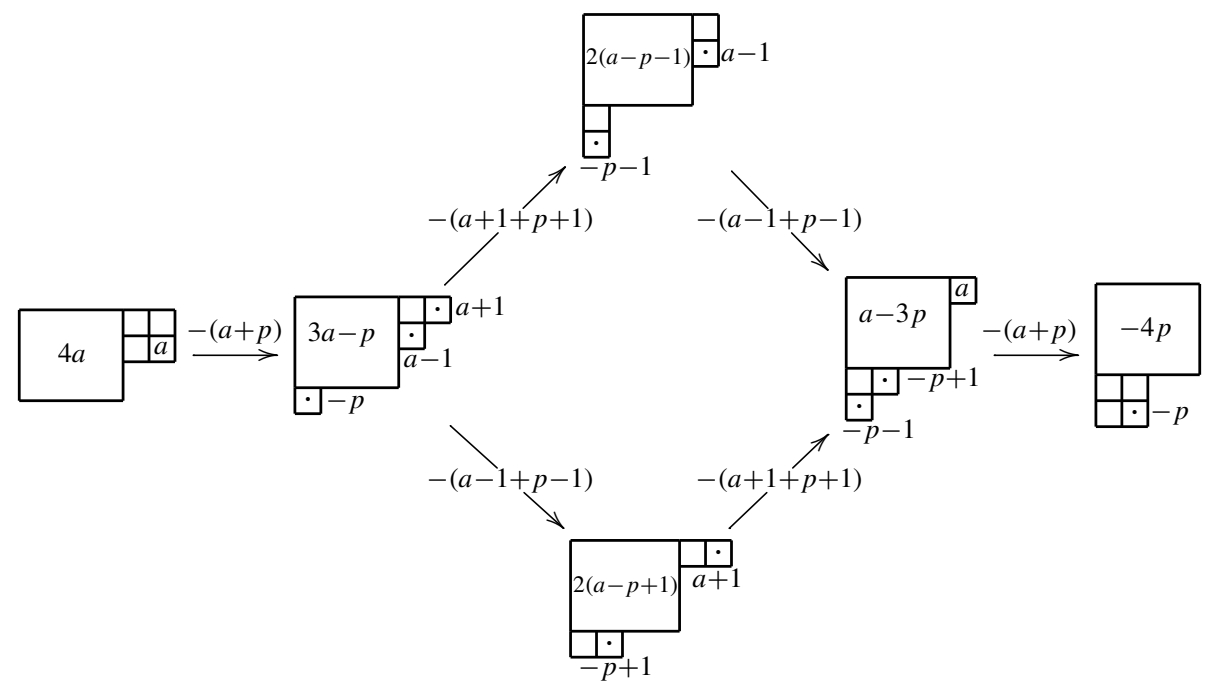

Figure 3. An illustration of Lemmas 4.4 and 4.5: The process of constructing partitions in $\mathscr{P}$, those partitions indexing nontrivial components of $L\left(\left(a^{p}\right)\right) \otimes L\left(\left(b^{q}\right)\right)$. In this example, $a, p \geq 2$ and $b=q=2$. The leftmost partition is $\left(a^{p}\right)+\left(2^{2}\right)$. The larger outlined area represents $a \times p$ boxes. Partitions are labeled with the action of $\gamma$ in the case where $\mathfrak{g}=\mathfrak{g l}_{n}$. Edges represent a box in the leftmost partition being moved down to its lower complementary position (as described in Remark 4.3) to form the rightmost partition, and are labeled by the change this presents in the value of $\gamma$. Boxes are marked if they are a change to the left or right, and are labeled by their contents.

placed some dots in the figure

(1) $\mu$ has height $p+q+1$ : In this case, exactly one box can be removed to form a partition that satisfies (4-5), the box in position $(1, p+q+1)$. This partition $\mu$ looks like the partition in Figure 4 with only box 1 added. For example

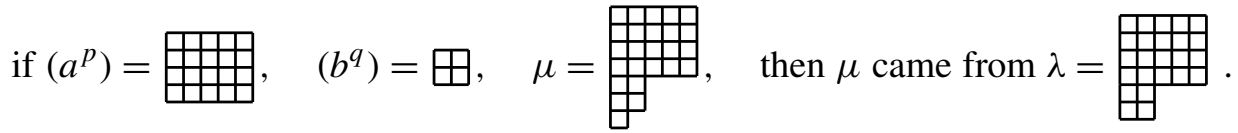

(2) $\mu_{q+1}=a+1$ : In this case, there is exactly one box that can be removed to obtain a partition that satisfies (4-5), the box in position $(a+1, q+1)$. This partition $\mu$ looks like the partition in Figure 4 with only box 2 added. 

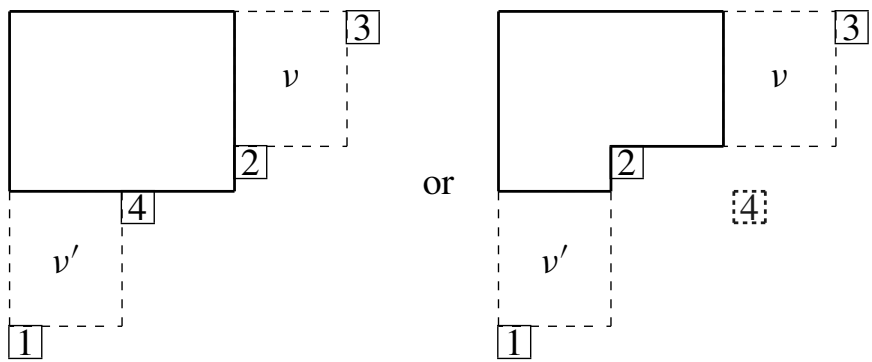

Figure 4. Added boxes corresponding to partitions with one parent, as described in cases 1-4 in the proof of Lemma 4.6 (see also Figure 2). At left, the case $a>b$. At right, the case $a<b$.

(3) $\mu_{1}=a+b+1$ : Again, there is exactly one box that can be removed, the box in position $(a+b+1,1)$. This partition $\mu$ looks like the partition in Figure 4 with only box 3 added.

(4) $\mu_{p+1}=b+1$ : This is similar to the case above, but is a little more complex. We can only see $\mu_{p+1}=b+1$ when $a>b$ and $\mu_{q}=a$. So the only removable box is the one in position $(b+1, p+1)$. This partition $\mu$ looks like the partition in Figure 4 with only box 4 added.

(5) $\mu_{j}+\mu_{p+q-j+1}=a+b+1$ for some $1 \leq j \leq p$, but $\mu_{j}<a+b+1$ and $\mu_{p+q-j+1}<\min (a, b)+1$ : This is the case which will yield two partitions. One is the partition in which we remove the box in position $\left(\mu_{j}, j\right)$; the other is the partition in which we remove the box in position $\left(a+b+1-\mu_{j}\right.$, $p+q+1-j$ ). This partition $\mu$ looks like those in Figure 5, where the boxes marked $x$ and $y$ are corner boxes, one of $x$ or $y$ has position $(i, j)$, and the other has position $\left(a+b+1-\mu_{j}, p+q+1-j\right)$.

For example,

if $\left(a^{p}\right)=\bigoplus, \quad\left(b^{q}\right)=\boxplus, \quad$ and $\quad \mu=\prod^{\prime}$,

then $\mu$ came from $\lambda=\bigoplus^{\text {or }} \lambda=\prod^{\text {. }}$.

Lemma 4.7. Suppose $\mu \in \mathscr{P}_{1}$ and $\lambda \in \mathscr{P}$ differ by a box, and $c(\mu / \lambda)$ is the content of this box. Then

(1) there is exactly one such $\lambda$ if and only if $c(\mu / \lambda)$ is equal to $-p-q, a-q$, $a+b$, or $b-p$, and 

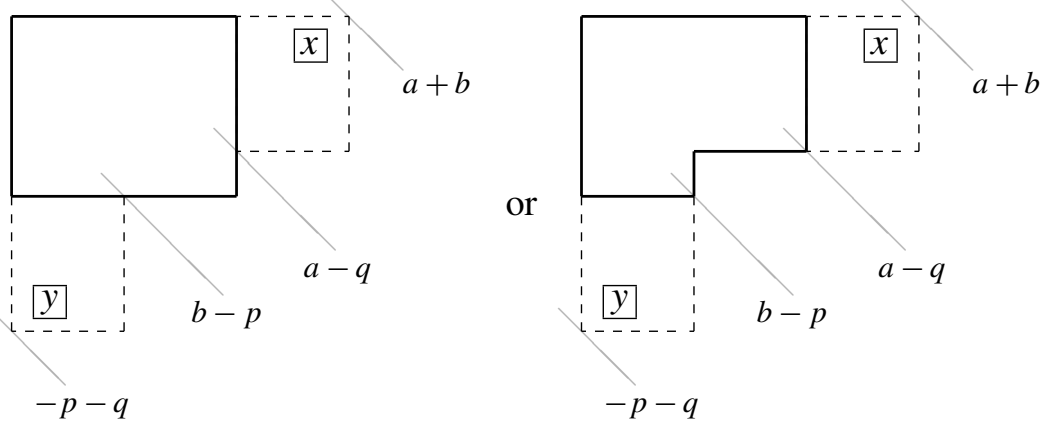

Figure 5. Added boxes corresponding to partitions with two parents, as described in case 5 in the proof of Lemma 4.6 (see also Figure 2). At left, the case $a>b$. At right, the case $a<b$. Critical diagonals are marked with contents $a+b, a-q, b-p$, and $-p-q$ for Lemma 4.7.

(2) if $c(\mu / \lambda)$ is not equal to $-p-q, a-q, a+b$, or $b-p$, then there is exactly one $\lambda^{\prime} \in \mathscr{P}$ distinct from $\lambda$ that differs from $\mu$ by a box, and

$$
c\left(\mu / \lambda^{\prime}\right)=a-p+b-q-c(\mu / \lambda) .
$$

Proof. If $\mu \in \mathscr{P}_{1}$ satisfies cases $1-4$ in Lemma 4.6, then

$$
c(\mu / \lambda)=-p-q, a-q, a+b, \text { or } b-p .
$$

The final case yielded two partitions that differ by the movement of one box. If a box in position $(i, j)$ in $\lambda \in \mathscr{P}$ can be moved to get another partition in $\mathscr{P}$, then that box must satisfy either

(i) $\max (a, b)<i \leq a+b$ and $0<j \leq q$, or

(ii) $0<i \leq \min (a, b)$ and $p<j \leq p+q$.

If $(i, j)$ satisfies $(i)$, then

$$
\max (a, b)-q<i-j<a+b-q \text {. }
$$

So since $p \geq q$,

$$
-p-q<i-j, \quad a-q<i-j, \quad b-p<i-j, \quad \text { and } \quad i-j<a+b \text {. }
$$

If $(i, j)$ satisfies (ii), then

$$
-p-q<i-j<\min (a, b)-p \text {. }
$$


So, similarly,

$$
-p-q<i-j, \quad i-j<a-q, \quad i-j<b-p \quad \text { and } \quad i-j<a+b .
$$

Thus, if there are two partitions in $\mathscr{P}$ that can be obtained by removing a box from $\mu$, then the contents of those boxes are distinct from $-p-q, a-q, a+b$, and $b-p$. See Figure 5 for an illustration of these bounds.

4.2. Action on tensor space. We return now to the representation

$$
\Phi: \mathscr{G}_{k} \rightarrow \operatorname{End}_{\mathfrak{g}}\left(M \otimes N \otimes V^{\otimes k}\right)
$$

in Theorem 2.1. Unfortunately, this map does not factor through the quotient defined in (3-1) and (3-2). However, we can twist by the following automorphism of $\mathscr{G}_{k}$ to acquire the desired representations of $\mathscr{H}_{k}^{\text {ext }}$.

Lemma 4.8. Fix $c_{x}, c_{y}, c_{z}, d \in \mathbb{C}$. The map $\phi: \mathscr{G}_{k} \rightarrow \mathscr{G}_{k}$ given by

$$
\begin{aligned}
x_{i} & \mapsto x_{i}+\left((i-1) d+c_{x}\right), & t_{s_{i}} \mapsto t_{s_{i}}, \\
y_{i} & \mapsto y_{i}+\left((i-1) d+c_{y}\right), & z_{0} \mapsto z_{0}+c_{z}, \\
z_{i} \mapsto z_{i}+\left((i-1) d+c_{x}+c_{y}\right), & &
\end{aligned}
$$

is an algebra automorphism.

Proof. Observe that for $i \geq 1$,

$$
\begin{aligned}
& \phi\left(x_{i+1}-t_{s_{i}} x_{i} t_{s_{i}}\right)=x_{i+1}-t_{s_{i}} x_{i} t_{s_{i}}+d, \\
& \phi\left(y_{i+1}-t_{s_{i}} y_{i} t_{s_{i}}\right)=y_{i+1}-t_{s_{i}} y_{i} t_{s_{i}}+d, \quad \phi\left(m_{i}\right)=m_{i}+(i-1) d .
\end{aligned}
$$

Relations (2-10), (2-11), and (2-4) follow directly.

Reviewing notation from Section 2.1, we denote by $\gamma_{M, N}$ the operator that acts on $M$ and $N$ in $M \otimes N \otimes V^{\otimes k}$ by $\gamma$ and on all other factors by the identity. Similarly, $\gamma_{i, j}$ acts by $\gamma$ on $i$-th and $j$-th factors of $V$, and for a factor $X, \gamma_{X, i}$ acts on $X$ and the $i$-th copy of $V, \kappa_{X}$ acts by $\kappa$ on $X$, and $\kappa_{X, \leq i}$ acts by $\kappa$ on $X$ and the first $i$ factors of $V$ (where $\kappa_{X, \leq 0}=\kappa_{X}$ ).

Now, define

$$
\Phi^{\prime}=\Phi \circ \phi: \mathscr{G}_{k} \rightarrow \operatorname{End}_{\mathfrak{g}}\left(M \otimes N \otimes V^{\otimes k}\right),
$$


so that

$$
\begin{aligned}
& \Phi^{\prime}\left(x_{i}\right)=\frac{1}{2}\left(\kappa_{M, \leq i}-\kappa_{M, \leq i-1}\right)+\left((i-1) d+c_{x}\right) \mathrm{id}, \\
& \Phi^{\prime}\left(y_{i}\right)=\frac{1}{2}\left(\kappa_{N, \leq i}-\kappa_{N, \leq i-1}\right)+\left((i-1) d+c_{y}\right) \mathrm{id}, \\
& \Phi^{\prime}\left(z_{i}\right)=\frac{1}{2}\left(\kappa_{M \otimes N, \leq i}-\kappa_{M \otimes N, \leq i-1}+\kappa_{V}\right)+\left((i-1) d+c_{x}+c_{y}\right) \mathrm{id} \\
& \quad \text { for } 1 \leq i \leq k, \\
& \Phi^{\prime}\left(z_{0}\right)=\frac{1}{2}\left(\kappa_{M \otimes N}-\kappa_{M}-\kappa_{N}\right)+c_{z} \mathrm{id}=\gamma_{M, N}+c_{z} \mathrm{id}, \\
& \Phi^{\prime}\left(t_{s_{j}}\right)=\mathrm{id}_{M} \otimes \mathrm{id}_{N} \otimes \mathrm{id}_{V}^{\otimes(j-1)} \otimes s \otimes \mathrm{id}_{V}^{\otimes(k-j-1)}, \\
& \quad \text { where } s \cdot(u \otimes v)=v \otimes u \text { for } 1 \leq j \leq k-1 .
\end{aligned}
$$

Theorem 4.9. Fix $\mathfrak{g}=\mathfrak{g l}_{n}$ or $\mathfrak{s l}_{n}$, and let $M=L\left(\left(a^{p}\right)\right), \quad N=\left(\left(b^{q}\right)\right)$, and $V=$ $L\left(\omega_{1}\right)$.

(a) When $\mathfrak{g}=\mathfrak{g l}_{n}$, fix $c_{x}=c_{y}=-\frac{1}{2} n$, and $d=0$.

(b) When $\mathfrak{g}=\mathfrak{s l}_{n}$, fix

$$
c_{x}=\frac{a p}{n}-\frac{1}{2}\left(n-\frac{1}{n}\right) \quad c_{y}=\frac{b q}{n}-\frac{1}{2}\left(n-\frac{1}{n}\right), \quad d=\frac{1}{n} .
$$

For these $c_{x}, c_{y}$, and $d$, and any choice of $c_{z}$, the map $\Phi^{\prime}$ in (4-6) factors through the quotient by (3-1) and (3-2), and so defines an action of $\mathcal{H}_{k}^{\text {ext }}$ that commutes with the action of $\mathfrak{g}$.

Proof. The relations in (3-1) can be rewritten as

$$
x_{i+1}-t_{s_{i}} x_{i} t_{s_{i}}=t_{s_{i}}, \quad y_{i+1}-t_{s_{i}} y_{i} t_{s_{i}}=t_{s_{i}} \quad \text { for } i=1, \ldots, k-1 .
$$

Recall from (2-16) that for $X=M, N$, or $M \otimes N$,

$$
\kappa_{X, \leq j}=\kappa_{X}+j \kappa_{V}+2\left(\sum_{1 \leq i \leq j} \gamma_{X, i}+\sum_{1 \leq r<s \leq j} \gamma_{r, s}\right)
$$

and so

$$
\kappa_{X, \leq i}-\kappa_{X, \leq i-1}=\kappa_{V}+2 \gamma_{X, i}+2 \sum_{1 \leq \ell<i} \gamma_{\ell, i}
$$

as an operator on $X \otimes V^{\otimes k}$. Therefore

$$
\begin{aligned}
& \left(\kappa_{X, \leq i+1}-\kappa_{X, \leq i}\right)-s_{i}\left(\kappa_{X, \leq i}-\kappa_{X, \leq i-1}\right) s_{i} \\
& \quad=\kappa_{V}+2 \gamma_{X, i+1}+2 \sum_{1 \leq \ell<i+1} \gamma_{\ell, i+1}-s_{i}\left(\kappa_{V}+2 \gamma_{X, i}+2 \sum_{1 \leq \ell<i} \gamma_{\ell, i}\right) s_{i} \\
& \quad=\kappa_{V}+2 \gamma_{X, i+1}+2 \sum_{1 \leq \ell<i+1} \gamma_{\ell, i+1}-\left(\kappa_{V}+2 \gamma_{X, i+1}+2 \sum_{1 \leq \ell<i} \gamma_{\ell, i+1}\right) \\
& \quad=2 \gamma_{i, i+1} .
\end{aligned}
$$


This means that to show (3-1), it only remains to be checked that $\mathrm{id}_{M} \otimes \mathrm{id}_{N} \otimes \mathrm{id}_{V}^{\otimes i-1} \otimes s \otimes \mathrm{id}_{V}^{\otimes k-i-1}$

$$
\begin{aligned}
& =\Phi^{\prime}\left(t_{s_{i}}\right)=\Phi^{\prime}\left(x_{i+1}-t_{s_{i}} x_{i} t_{s_{i}}\right) \\
& =\frac{1}{2}\left(\left(\kappa_{M, \leq i+1}-\kappa_{M, \leq i}\right)+2\left(i d+c_{x}\right)\right. \\
& \qquad \gamma_{i, i+1}+d= \begin{cases}\left.\gamma_{i, i+1}\left(\kappa_{M, \leq i}-\kappa_{M, \leq i-1}+2\left((i-1) d+c_{x}\right)\right) s_{i}\right) \\
\gamma_{i, i+1}+1 / n & \text { when } \mathfrak{g}=\mathfrak{g l}_{n},\end{cases}
\end{aligned}
$$

as operators on $M \otimes N \otimes V^{\otimes k}$ (the check for $\Phi^{\prime}\left(t_{s_{i}}\right)=\Phi^{\prime}\left(y_{i+1}-t_{s_{i}} y_{i} t_{s_{i}}\right)$ is the same).

The decomposition of $V \otimes V$ is

$$
V \otimes V=L(\square \square) \oplus L(\square)
$$

where if $v_{1}, \ldots, v_{n}$ is a basis for $V$, then

$$
\begin{aligned}
L(\square) & =\operatorname{span}_{\mathbb{C}}\left\{v_{i} \otimes v_{j}+v_{j} \otimes v_{i} \mid 1 \leq i, j \leq n\right\}, \quad \text { and } \\
L(\square) & =\operatorname{span}_{\mathbb{C}}\left\{v_{i} \otimes v_{j}-v_{j} \otimes v_{i} \mid 1 \leq i, j \leq n\right\} .
\end{aligned}
$$

It follows from this decomposition and Lemma 4.2 that the actions of $s$ and $\gamma$ are given by
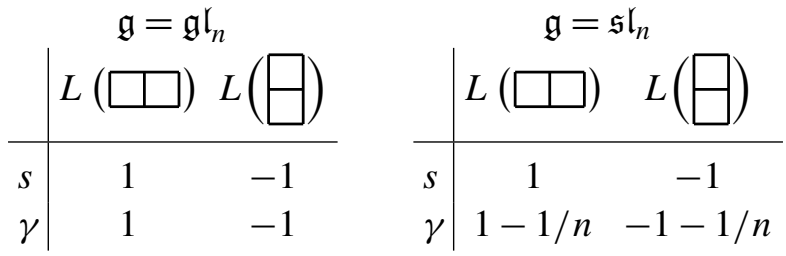

so (3-1) is satisfied.

Next we check $\left(x_{1}-a\right)\left(x_{1}+p\right)=0$. By (4-7), we have

$$
\Phi^{\prime}\left(x_{1}\right)=\frac{1}{2} \kappa_{V}+\gamma_{M, 1}+c_{x} .
$$

The module $M \otimes V$ decomposes as

$$
M \otimes V=L\left(\begin{array}{ll}
a & p
\end{array}\right) \oplus L\left(\begin{array}{l}
a \\
p
\end{array}\right) .
$$

Case $1\left(\mathfrak{g}=\mathfrak{g l}_{n}\right)$. By Lemma 4.1,

$$
\kappa_{V}=\left\langle\omega_{1}, \omega_{1}+2 \delta\right\rangle-(n-1)\left|\omega_{1}\right|=1+(n-1)-(n-1)=n,
$$


so $\kappa_{V} / 2+c_{x}=0$. By Lemma 4.2 and the decomposition in (4-8), $\gamma_{M, 1}$ is equal to $a$ or $-p$, so $\Phi^{\prime}\left(x_{1}-a\right)\left(x_{1}+p\right)=0$ as desired.

Case $2\left(\mathfrak{g}=\mathfrak{s l}_{n}\right)$. By Lemma 4.1,

$$
\kappa_{V}=\left\langle\omega_{1}, \omega_{1}+2 \rho\right\rangle=n-1 / n,
$$

so $\kappa_{V} / 2+c_{x}=a p / n$. By Lemma 4.2 and the decomposition in (4-8), $\gamma_{M, 1}$ is equal to $(a-a p / n)$ or $(-p-a p / n)$ so $\Phi^{\prime}\left(x_{1}-a\right)\left(x_{1}+p\right)=0$ as desired.

The relation $\left(y_{1}-b\right)\left(y_{1}+q\right)=0$ follows analogously, and so (3-2) is satisfied.

\subsection{Bratteli diagrams and seminormal bases. Let}

$$
\mathbb{C}=\mathscr{C}_{0} \subseteq \mathscr{b}_{1} \subseteq \mathscr{b}_{2} \subseteq \cdots
$$

be a chain of semisimple algebras. Let $\hat{\mathscr{C}}_{k}$ be the set of equivalence classes of finitedimensional irreducible $\mathscr{C}_{k}$-modules for $k=0,1, \ldots$, and write $\mathscr{C}^{\mu}$ for a module in the class $\mu \in \hat{\mathscr{C}}_{k}$. Here we will be describing an oriented ranked graph, the Bratteli diagram for (4-11), which encodes the representation theory of $\mathscr{C}_{k}$ in terms of the representation theory of $\mathscr{C}_{i}$ for $i<k$. In the example where $\mathscr{C}_{k}$ is the group algebra of the symmetric group $\mathbb{C} S_{k}$, this diagram the same as Young's diagram. This exposition on Bratteli diagrams and seminormal bases follows closely Okounkov and Vershik's discussion [1996] of chains of finite-dimensional semisimple associative algebras in general.

The Bratteli diagram associated to a chain (4-11) is an oriented ranked graph, with a rank for each $\mathscr{C}_{i}$. The vertices of rank or on level $k$ are the elements of the set $\hat{\mathscr{C}}_{k}$. Two vertices $\mu \in \hat{\mathscr{C}}_{k-1}$ and $v \in \hat{\mathscr{C}}_{k}$ are joined by $d$ oriented edges from $\mu$ to $v$ if

$$
d=\operatorname{dim} \operatorname{Hom}_{\mathscr{C}_{k-1}}\left(\mathscr{C}^{\mu}, \mathscr{C}^{\nu}\right),
$$

that is, $d$ is the multiplicity of $\mathscr{C}^{\mu}$ in the restriction of $\mathscr{C}^{v}$ to a $\mathscr{C}_{k-1}$-module. Write $\mu \searrow v$ if $\mu$ and $v$ are connected by an edge from $\mu$ to $v$.

If $\mu \in \hat{\mathscr{C}}_{i}$ and $\lambda \in \hat{\mathscr{C}}_{k}$ with $i<k$, write

$\mu \subset \lambda \quad$ if there is a path $\mu \searrow \cdots \searrow \lambda$ from $\mu$ to $\lambda$ in the Bratteli diagram.

In other words, $\mu \subset \lambda$ if and only if the multiplicity of $\mu$ in $\lambda$ after appropriate restriction is nonzero.

Our favorite examples are when $\mathfrak{g}=\mathfrak{s l}_{n}$ or $\mathfrak{g l}_{n}, M$ and $N$ are finite-dimensional simple modules indexed by rectangular partitions, $V$ is the standard representation, and

(1) $\mathscr{C}_{k}=\operatorname{End}_{\mathfrak{g}}\left(M \otimes V^{\otimes k}\right)$, 
(2) $\mathscr{C}_{k}=\operatorname{End}_{\mathfrak{g}}\left(N \otimes V^{\otimes k}\right)$, or

(3) $\mathscr{C}_{0}=\operatorname{End}_{\mathfrak{g}}(M)$ and $\mathscr{C}_{k}=\operatorname{End}_{\mathfrak{g}}\left(M \otimes N \otimes V^{\otimes k-1}\right)$.

In fact, as we will see in Examples 4.3 and 4.4, these Bratteli diagrams are all multiplicity free ( $d$ in (4-12) is always 0 or 1$)$. With multiplicity free diagrams, the decomposition

$$
\mathscr{C}^{\lambda}=\bigoplus_{\substack{\mu \in \hat{\mathbb{E}}_{k-1} \\ \mu \lambda \lambda}} \mathscr{C}^{\mu}
$$

is canonical. By induction, we obtain a canonical decomposition of the module $\mathscr{C}^{\lambda}$ into irreducible one-dimensional $\mathscr{C}_{0}$-modules

$$
\mathscr{C}^{\lambda}=\bigoplus_{T} \mathbb{C} v_{T}
$$

indexed by all possible paths

$$
T=\left(T^{(0)} \searrow T^{(1)} \searrow \cdots \searrow T^{(k)}=\lambda\right),
$$

where $T^{(i)} \in \hat{\mathscr{C}}_{i}$ for each $0 \leq i \leq k$. In particular, $v_{T}$ is the unique element (up to scalar multiplication) respecting the inductive process, that is, for each $0 \leq i \leq k$, after the induction

$$
\operatorname{Ind}_{\mathscr{C}_{0}}^{\mathscr{C}_{i}} \bigoplus_{T} \mathbb{C} v_{T}=\bigoplus_{\nu} c_{\nu} \mathscr{C}^{v}
$$

each vector $v_{T}$ lands in the isotypic component $c_{T^{(i)}} \mathscr{C}^{T^{(i)}}$. We call the basis $\left\{v_{T}\right\}$ of $\mathscr{C}^{\lambda}$ a (nonnormalized) seminormal basis.

Any finite dimensional $\mathfrak{g}$-module $U$ decomposes as a $\left(\mathfrak{g}, \operatorname{End}_{\mathfrak{g}}(U)\right)$-bimodule as

$$
U \cong \bigoplus_{\lambda} L(\lambda) \otimes \mathscr{L}^{\lambda}
$$

where $\mathscr{L}^{\lambda}$ are distinct irreducible $\operatorname{End}_{\mathfrak{g}}(U)$-modules (see [Goodman and Wallach 1998, Theorem 3.3.7]) and are isomorphic to the span of all highest weight vectors of weight $\lambda$ in $U$. So both the irreducible $\mathfrak{g}$-modules and the irreducible $\operatorname{End}_{\mathfrak{g}}\left(M \otimes N \otimes V^{\otimes k}\right)$-modules appearing in $M \otimes N \otimes V^{\otimes k}$ are indexed by the same set. Therefore, the result of the combinatorics outlined in Section 4.1 is that, for our favorite examples, the paths in (4-13) are in bijection with specific sets of tableaux, which we define now.

For two partitions $\lambda \subseteq \mu$, the skew shape $\mu / \lambda$ is the portion of $\mu$ not contained in $\lambda$. A (standard) $\mu / \lambda$-tableaux is a filling of the skew shape $\mu / \lambda$ with the integers $1, \ldots,|\mu|-|\lambda|$ so that the row fillings increase from left to right and the column fillings increase from top to bottom. For example, if

$$
\lambda=\square \text { and } \mu=\square \text {, }
$$


then there are three $\mu / \lambda$-tableaux,

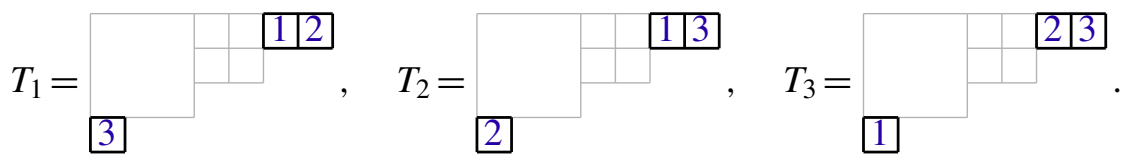

Now consider sequences of partitions $T=\left(\lambda=T^{(0)} \searrow T^{(1)} \searrow \cdots \searrow T^{(k)}=\mu\right)$, where $T^{(i)}$ is obtained from $T^{(i-1)}$ by adding a box. We can identify each $T$ with the $\mu / \lambda$-tableaux built by placing the integer $i$ in the box added at the $i$-th step. For example,

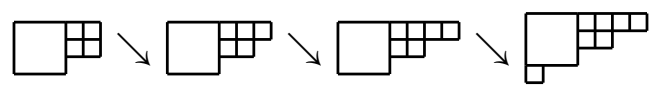

is identified with $T_{1}$ in (4-15).

Example 4.3 (Bratteli diagram for $\operatorname{End}_{\mathfrak{g}}\left(M \otimes V^{\otimes k}\right)$ ). Let $\mathfrak{g}=\mathfrak{s l}_{n}$ or $\mathfrak{g l}_{n}$, let $M=$ $L\left(\left(a^{p}\right)\right)$ and $V=L\left(\left(1^{1}\right)\right)$, and consider the example where $\mathscr{C}_{k}=\operatorname{End}_{\mathfrak{g}}\left(M \otimes V^{\otimes k}\right)$. The inclusion map in (2-13) provides a chain

$$
\mathbb{C}=\operatorname{End}_{\mathfrak{g}}(M) \subseteq \operatorname{End}_{\mathfrak{g}}(M \otimes V) \subseteq \cdots
$$

as in Equation (4-11). By identifying classes of $\hat{\mathscr{C}}_{k}$ with partitions as in Section 4.1, we learn from Example 4.1 that the dimensions in (4-12) are all 0 or 1, and that the paths in (4-13) are in bijection with the set of tableaux

$$
\left\{T=\left(\left(a^{p}\right)=T^{(0)} \searrow \cdots \searrow T^{(k)}\right)\right\} .
$$

In particular, each $\mathscr{C}_{k}$-module $\mathscr{C}^{\lambda}$ has its seminormal basis $\left\{v_{T}\right\}$ indexed by the tableaux in (4-17) that end at $T^{(k)}=\lambda$. Moreover, $\mathscr{C}^{\lambda}$ is the same as $\mathscr{L}^{\lambda}$ in (4-14), and each $v_{T}$ is a highest weight vector of weight $T^{(i)}$ in $\operatorname{Res}_{\mathscr{C}_{i}}^{\ell_{k}} M \otimes V^{\otimes k}$ for each $i=0, \ldots, k$.

Example 4.4 (Bratteli diagram for $\operatorname{End}_{\mathfrak{g}}\left(M \otimes N \otimes V^{\otimes k}\right)$ ). Let $\mathfrak{g}=\mathfrak{s l}_{n}$ or $\mathfrak{g l}_{n}$, $M=L\left(\left(a^{p}\right)\right), N=L\left(\left(b^{q}\right)\right)$, and $V=L\left(\left(1^{1}\right)\right)$, and consider the example where

$$
\mathscr{C}_{0}=\operatorname{End}_{\mathfrak{g}}(M) \quad \text { and } \quad \mathscr{C}_{k}=\operatorname{End}_{\mathfrak{g}}\left(M \otimes N \otimes V^{\otimes k-1}\right) .
$$

Just as in the previous example, these $\mathscr{C}_{k}$ satisfy the chain

$$
\mathbb{C}=\operatorname{End}_{\mathfrak{g}}(M) \subseteq \operatorname{End}_{\mathfrak{g}}(M \otimes N) \subseteq \operatorname{End}_{\mathfrak{g}}(M \otimes N \otimes V) \subseteq \cdots .
$$

As in Example 4.2, if $\left(a^{p}\right)$ and $\left(b^{q}\right)$ are rectangular partitions, let $\mathscr{P}=\mathscr{P}\left(\left(a^{p}\right),\left(b^{q}\right)\right)$ be the set of partitions $\mu$ for which $L(\mu)$ appears as a submodule of $L\left(\left(a^{p}\right)\right) \otimes$ $L\left(\left(b^{q}\right)\right)$. In particular, each $L(\mu)$ appears with multiplicity 1 . Let $\mathscr{P}_{0}=\mathscr{P}$ and define $\mathscr{P}_{k}$ to be the set of partitions that are obtained by adding a box to an element of $\mathscr{P}_{k-1}$.

The classes in $\hat{\mathscr{C}}_{k}$ are in bijection with the partitions in $\mathscr{P}_{k-1}$, and the Bratteli diagram for the chain in (4-18) is the following oriented ranked graph: 


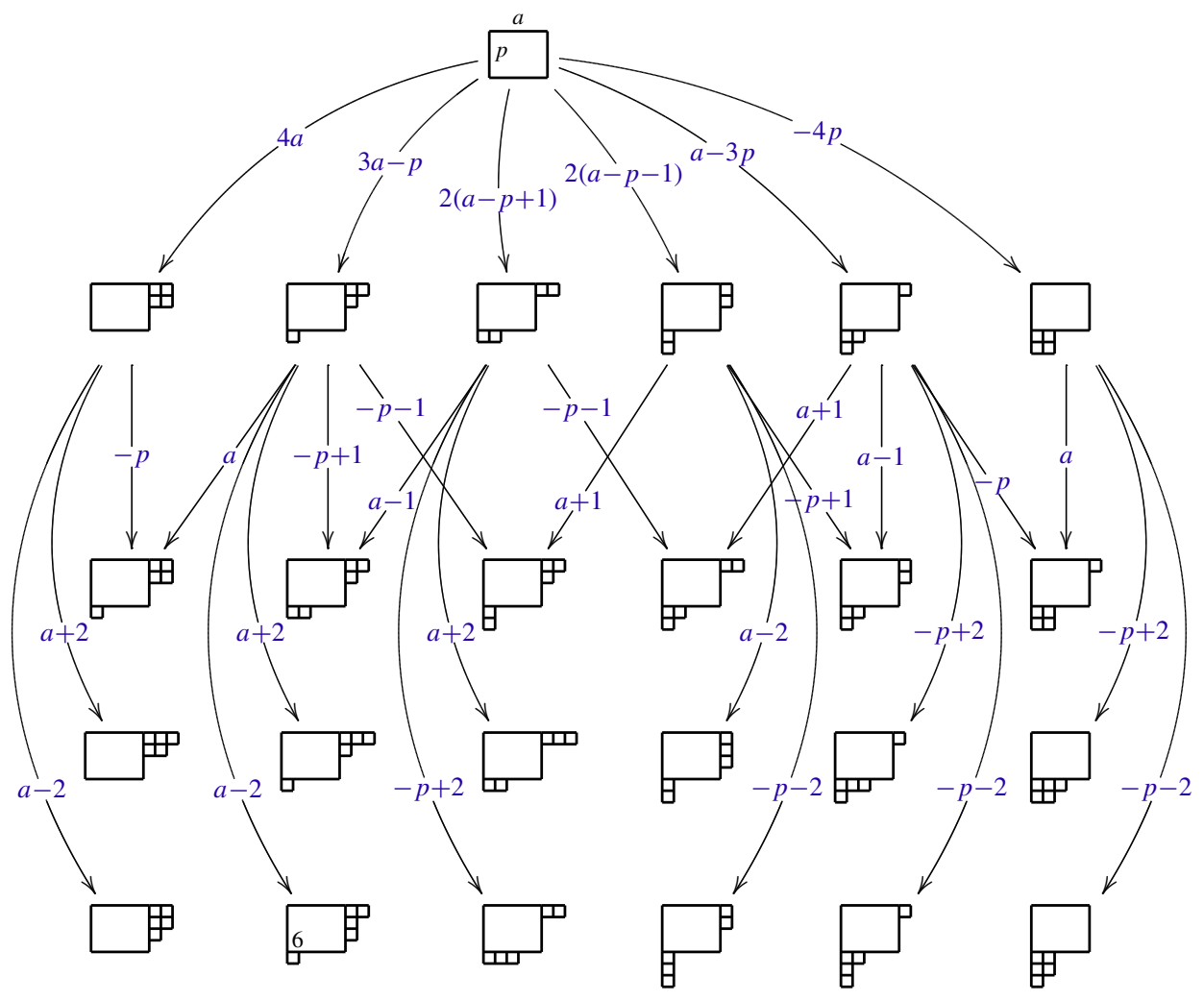

Figure 6. Levels 0,1 , and 2 of a Bratteli diagram encoding isotypic components of $M \otimes N \otimes V$. The edges are labeled by combinatorial values given by the action of $\mathcal{H}_{k}^{e x t}$ as stated later in Theorem 4.10.

Vertices: The vertices are labeled by partitions.

Level 0: On level 0, place one vertex, labeled by $\left(a^{p}\right)$.

Level $k>0$ : On level $k>0$, place one vertex for each partition in $\mathscr{P}_{k-1}$.

Edges: Edges connect two vertices on adjacent levels.

Connect the vertex on level 0 to each of the vertices on level 1 with one edge. Connect each vertex on level $k-1$ to a vertex on level $k$ if the vertex on level $k$ can be obtained by adding a box to the corresponding vertex on level $k-1$.

For the examples where $a, p>2$ and $b=q=2$, levels 0,1 , and 2 are shown of the Bratteli diagram in Figure 6. Notice that each of the partitions in $\mathscr{P}_{1}$ comes from exactly one or two partitions in $\mathscr{P}_{0}$; this happens for any choice of $a, b, p$, and $q$ by Lemma 4.6. 
Again, we learn from Examples 4.1 and 4.2 that the dimensions in (4-12) are all 0 or 1 , and that the paths in (4-13) are in bijection with the set of tableaux

$$
\left\{T=\left(T^{(1)} \searrow \cdots \searrow T^{(k)}\right) \mid T^{(i)} \in \mathscr{P}_{i-1} \text { for } i=1, \ldots, k\right\} .
$$

In particular, for each $\lambda \in \mathscr{P}_{k-1}$, the $\mathscr{C}_{k}$-module $\mathscr{C}^{\lambda}$ has seminormal basis $\left\{v_{T}\right\}$ indexed by the tableaux in (4-19) that end at $T^{(k)}=\lambda$. Moreover, $\mathscr{C}^{\lambda}$ is the same as $\mathscr{L}^{\lambda}$ in (4-14), and each $v_{T}$ is a highest weight vector of weight $T^{(i)}$ in $\operatorname{Res}_{\mathscr{C}_{i}}^{\ell_{k}} M \otimes$ $N \otimes V^{\otimes k-1}$ for each $i=0, \ldots, k-1$.

We now return to the representation $\Phi^{\prime}$ in Theorem 4.9, and combinatorially determine the eigenvalues of the operators $\Phi^{\prime}\left(x_{i}\right), \Phi^{\prime}\left(y_{i}\right)$, and $\Phi^{\prime}\left(z_{i}\right)$ for $i=1, \ldots, k$. If $\lambda \subset \mu$ are partitions differing by a box in column $c$ and row $r$, recall $c(\mu / \lambda)=c-r$ is the content of the box $\mu / \lambda$ in $\mu$.

Theorem 4.10. Let $\Phi^{\prime}: \mathscr{H}_{k}^{\text {ext }} \rightarrow \operatorname{End}_{\mathfrak{g}}\left(M \otimes N \otimes V^{\otimes k}\right)$ be the representation in Theorem 4.9, with

$$
c_{z}= \begin{cases}0 & \text { if } \mathfrak{g}=\mathfrak{g l}_{n}, \\ \text { abpq } / n & \text { if } \mathfrak{g}=\mathfrak{s l}_{n} .\end{cases}
$$

There is a basis $\left\{v_{T}\right\}$ of $M \otimes N \otimes V^{\otimes k}$ indexed by standard tableaux

$$
\left\{T=\left(T^{(0)} \searrow \cdots \searrow T^{(k)}\right) \mid T^{(j)} \in \mathscr{P}_{j} \text { for } j=0, \ldots, k\right\}
$$

with action

$$
\Phi^{\prime}\left(z_{i}\right) v_{T}=c\left(T^{(i)} / T^{(i-1)}\right) v_{T} \quad \text { for } i=1, \ldots, k,
$$

and

$$
\Phi^{\prime}\left(z_{0}\right) v_{T}=\left(a b q+2 \sum_{B \in \mathscr{P}_{T^{(0)}}}\left(c(B)-\frac{1}{2}(a-p+b-q)\right)\right) v_{T},
$$

where $\mathscr{B}_{\lambda}$ is the set of boxes in $\lambda$ in rows $p+1$ and below.

Proof. The basis for $M \otimes N \otimes V^{\otimes k}$ produced in Example 4.4 is specifically one that satisfies

$$
v_{T} \in v_{T^{(i)}} \otimes V^{\otimes(k-i)} \subseteq M \otimes N \otimes V^{\otimes k} \quad \text { for } i=0, \ldots, k,
$$

where $v_{T^{(i)}}$ is a highest weight vector of weight $T^{(i)}$ in $L\left(T^{(i)}\right) \subseteq M \otimes N \otimes V^{\otimes i}$. Therefore

$$
\begin{aligned}
\Phi^{\prime}\left(z_{i}\right) \cdot v_{T} & =\left(\frac{1}{2}\left(\kappa_{M \otimes N, \leq i}-\kappa_{M \otimes N, \leq i-1}+\kappa_{V}\right)+\left((i-1) d+c_{x}+c_{y}\right) \mathrm{id}\right) \cdot v_{T} \\
& =\left(\gamma_{L\left(T^{(i-1)}\right), V}+\kappa_{V}+\left((i-1) d+c_{x}+c_{y}\right) \mathrm{id}\right) v_{T} .
\end{aligned}
$$


By Lemma 4.2, (4-9) and (4-10),

$$
\begin{aligned}
\gamma_{L\left(T^{(i-1)}\right), V} & = \begin{cases}c\left(T^{(i)} / T^{(i-1)}\right) & \text { if } \mathfrak{g}=\mathfrak{g l}_{n}, \\
c\left(T^{(i)} / T^{(i-1)}\right)-(a p-b q+i-1) / n & \text { if } \mathfrak{g}=\mathfrak{s l}_{n}\end{cases} \\
\kappa_{V} & = \begin{cases}n & \text { if } \mathfrak{g}=\mathfrak{g l}_{n}, \\
n-1 / n & \text { if } \mathfrak{g}=\mathfrak{s l}_{n} .\end{cases}
\end{aligned}
$$

So since $c_{x}=c_{y}=-\frac{1}{2} n$ and $d=0$ when $\mathfrak{g}=\mathfrak{g l}_{n}$, and

$$
c_{x}=\frac{a p}{n}-\frac{1}{2}\left(n-\frac{1}{n}\right), c_{y}=\frac{b q}{n}-\frac{1}{2}\left(n-\frac{1}{n}\right), \text { and } d=\frac{1}{n} \quad \text { when } \mathfrak{g}=\mathfrak{s l}_{n},
$$

we have $\Phi^{\prime}\left(z_{i}\right) \cdot v_{T}=c\left(T^{(i)} / T^{(i-1)}\right) v_{T}$ as desired. Similarly, the action of $\Phi^{\prime}\left(z_{0}\right)$ follows from Lemma 4.5 .

Example 4.5. To illustrate, we apply Theorem 4.10 to the example where $a, p>2$, $b=q=2$, and $k=1$. Returning to Figure 6 above, we can read that there are eighteen distinct isotypic components of $M \otimes N \otimes V$, six of which correspond to 2-dimensional $\operatorname{End}_{\mathfrak{g}}(M \otimes N \otimes V)$-modules and twelve of which correspond to 1-dimensional End $\mathrm{E}_{\mathfrak{g}}(M \otimes N \otimes V)$-modules.

The edges connecting level 0 to level 1 are labeled by the combinatorial formula for the action of $z_{0}$, and the edges connecting level 1 to level 2 are labeled by the content of the box added. In general, we label the edges connecting level $i$ to level $i+1$ by the content of the box added. The paths in this diagram from $\left(a^{p}\right)$ to $\lambda \in \mathscr{P}_{1}$ index the basis of $\mathscr{L}^{\lambda}$, and $\Phi^{\prime}\left(z_{1}\right)$ and $\Phi^{\prime}\left(z_{0}\right)$ act on those basis elements by the corresponding edge labels.

Remark 4.11. Example 4.3 gives a basis $\left\{v_{T}\right\}$ of $M \otimes V^{\otimes k}$ indexed by standard tableaux

$$
\left\{T=\left(\left(a^{p}\right)=T^{(0)} \searrow \cdots \searrow T^{(k)}\right)\right\} .
$$

For every $n \in N$, there is a canonical map

$$
\iota_{n}: M \otimes V^{\otimes k} \hookrightarrow M \otimes V^{\otimes k} \otimes N \cong M \otimes N \otimes V^{\otimes k} .
$$

Therefore, by picking a basis $\left\{n_{j}\right\}$ of $N,\left\{v_{T}\right\}$ can be lifted to a basis $\left\{\iota_{n_{j}}\left(v_{T}\right)\right\}_{j, T}$ for $M \otimes N \otimes V^{\otimes k}$. A similar calculation as in Theorem 4.10 will produce

$$
\Phi^{\prime}\left(x_{i}\right) \cdot \iota_{n_{j}}\left(v_{T}\right)=c\left(T^{(i)} / T^{(i-1)}\right) \iota_{n_{j}}\left(v_{T}\right) .
$$

Similarly, there is a basis of highest weight vectors $\left\{v_{T}\right\}$ for $N \otimes V^{\otimes k}$ indexed by standard tableaux

$$
\left\{T=\left(\left(b^{q}\right)=T^{(0)} \searrow \cdots \searrow T^{(k)}\right)\right\} .
$$


By picking a basis $\left\{m_{j}\right\}$ for $M$, the map

$$
\iota_{m}: N \otimes V^{\otimes k} \hookrightarrow M \otimes N \otimes V^{\otimes k}
$$

produces a basis $\left\{\iota_{m_{j}}\left(v_{T}\right)\right\}_{j, T}$ that satisfies

$$
\Phi^{\prime}\left(y_{i}\right) \cdot \iota_{m_{j}}\left(v_{T}\right)=c\left(T^{(i)} / T^{(i-1)}\right) \iota_{m_{j}}\left(v_{T}\right) .
$$

These two examples reflect the fact that the degenerate two-boundary braid algebras and Hecke algebras contain one-boundary analogs (though this isomorphic containment is left for future work). Later, Theorem 5.3 will provide explicit formulas for $x_{1}$ (and therefore $y_{1}$ ) in terms of the basis given in Theorem 4.10, but we can already ascertain the eigenvalues of $x_{1}$ and $y_{1}$, as they are stable under a change of basis.

Remark 4.12. The algebras in (4-11) are also known as Gelfand-Zetlin algebras, and the seminormal bases are (nonnormalized) Gelfand-Zetlin bases. Denote the center of $\mathscr{C}_{i}$ by $Z\left(\mathscr{C}_{i}\right)$. The commutative subalgebra $\mathscr{A}_{k} \subseteq \mathscr{C}_{k}$ generated by the subalgebras $Z\left(\mathscr{C}_{0}\right), Z\left(\mathscr{C}_{1}\right), \ldots, Z\left(\mathscr{C}_{k}\right)$ is called the Gelfand-Zetlin subalgebra in [Okounkov and Vershik 1996]. It remains for future work to show that the subalgebra of $\operatorname{End}\left(M \otimes N \otimes V^{\otimes k}\right)$ generated by $\Phi^{\prime}\left(z_{0}\right), \Phi^{\prime}\left(z_{1}\right), \ldots, \Phi^{\prime}\left(z_{k}\right)$ has large index inside of the Gelfand-Zetlin subalgebra for the chain in Example 4.4. However, Theorem 4.10 is suggestive of this relationship, and future work on the center of $\mathscr{H}_{k}^{\text {ext }}$ will show that the subalgebra generated by subalgebras $Z\left(\mathscr{H}_{0}^{\text {ext }}\right), Z\left(\mathscr{H}_{1}^{\text {ext }}\right)$, $\ldots, Z\left(\mathscr{H}_{k}^{\text {ext }}\right)$, is in fact the same as the subalgebra generated by $z_{0}, z_{1}, \ldots, z_{k}$.

\section{Seminormal representations of $\mathscr{H}_{k}^{e x t}$}

Section 4.2 showed that a quotient of $\mathscr{H}_{k}^{\text {ext }}$ is a subalgebra of $\operatorname{End}_{\mathfrak{g}}\left(M \otimes N \otimes V^{\otimes k}\right)$, when $\mathfrak{g}=\mathfrak{g l}_{n}$ or $\mathfrak{s l}_{n}, \quad M$ and $N$ are simple $\mathfrak{g}$-modules indexed by rectangular partitions, and $V$ is the standard representation. Section 4.3 then showed that the action of the generators $z_{0}, \ldots, z_{k}$ on $M \otimes N \otimes V^{\otimes k}$ is simultaneously diagonalizable with eigenvalues given by combinatorial values. In this section, we study all seminormal representations, and conclude finally in Corollary 5.5 that the simple End $_{\mathfrak{g}}\left(M \otimes N \otimes V^{\otimes k}\right)$-modules in $M \otimes N \otimes V^{\otimes k}$ are also simple as $\mathscr{H}_{k}$-modules.

This section serves as a culmination of work done so far on $\mathscr{H}_{k}$ and $\mathscr{H}_{k}^{\text {ext }}$, and will draw on many results throughout the paper. We will be primarily citing results from Sections 3, 4.1 and 4.3. The presentation of choice for $\mathcal{H}_{k}^{\text {ext }}$ is given in Theorem 3.2; in particular, we switch from the generating set

$$
z_{0}, z_{1}, \ldots, z_{k}, x_{1}, \ldots, x_{k}, y_{1}, \ldots, y_{k}, t_{s_{1}}, \ldots, t_{s_{k-1}}
$$

to the generating set

$$
w_{0}, w_{1}, \ldots, w_{k}, x_{1}, t_{s_{1}}, \ldots, t_{s_{k-1}}
$$


where $w_{i}=z_{i}-(a-p+b-q) / 2$. Section 4.3 (specifically Example 4.4) introduces the combinatorial backbone of the modules that we study in this section; the bases for the modules in Proposition 5.2 are indexed by the same tableaux as in the Bratteli diagram for $\operatorname{End}_{\mathfrak{g}}\left(M \otimes N \otimes V^{\otimes k}\right)$ in Example 4.4. The specific combinatorial properties of these tableaux begin in Section 4.1: Example 4.2 describes the set $\mathscr{P}$ of partitions that index the simple submodules of $L\left(\left(a^{p}\right)\right) \otimes L\left(\left(p^{q}\right)\right)$, and Figure 2 provides a useful illustration of the partitions in $\mathscr{P}$; then Lemmas 4.6 and 4.7 tell us about the shape and symmetries of the Bratteli diagram at levels 0-2, and Figure 5 illustrates this symmetry. Finally, in Section 4.3, Theorem 4.10 tells us the correct action of the $w_{i}$, and Remark 4.11 tells us how to anticipate the eigenvalues of the action of $x_{1}$.

Fix $a, b, p, q$ nonnegative integers with $q \leq p$. Recall from Example 4.4 that $\mathscr{P}_{0}=\mathscr{P}$ is the set of partitions indexing simple submodules of $L\left(\left(a^{p}\right)\right) \otimes L\left(\left(b^{q}\right)\right)$, and $\mathscr{P}_{i}$ is the set of partitions obtained by adding a box to any partition in $\mathscr{P}_{i-1}$. Let $\mathscr{T}_{\lambda}$ be the set of tableaux

$$
\mathscr{T}_{\lambda}=\left\{T=\left(T^{(0)} \searrow \cdots \searrow T^{(k)}=\lambda\right) \mid T^{(0)} \in \mathscr{P}, T^{(i)} \in \mathscr{P}_{i}\right\} .
$$

The box added to $T^{(i-1)}$ to get $T^{(i)}$ is $b_{i}=T^{(i)} / T^{(i-1)}$. Define shifted contents

$$
\begin{aligned}
& c_{T}(0)=a b q-\left(\left|\Re_{\mu}\right|+\frac{1}{2}\right)(a-p+b-q)+2 \sum_{B \in \mathscr{B}_{\mu}} c(B), \\
& c_{T}(i)=c\left(T^{(i)} / T^{(i-1)}\right)-\frac{1}{2}(a-p+b-q),
\end{aligned}
$$

where $\mathscr{B}_{\mu}$ is the set of boxes in $\mu$ in rows $p+1$ and below, as described in Lemma 4.5.

Lemma 5.1. A tableau $T \in \mathscr{T}_{\lambda}$ is fully determined by $c_{T}(1), \ldots, c_{T}(k)$ and $T^{(k)}$.

Proof. This can be shown by induction on $k$. The key observation is that the value $c_{T}(i)$ for $i>0$ determines the diagonal on which $T^{(i)} / T^{(i-1)}$ lies. In any given partition, there is at most one removable box on any diagonal. So $c_{T}(k)$ and $T^{(k)}$ determines $T^{(k-1)}$. By iterating, $c_{T}(i)$ and $T^{(i)}$ determines $T^{(i-1)}$, so we can recover $T^{(k-1)}, T^{(k-2)}, \ldots, T^{(0)}$.

Two consecutive boxes $b_{i}$ and $b_{i+1}$ are in the same row or column if and only if $c\left(b_{i}\right)=c\left(b_{i+1}\right) \pm 1$. So for any $i$ for which $c_{T}(i) \neq c_{T}(i+1) \pm 1$, we can define $s_{i} T^{(i)}$ as the partition obtained removing $b_{i}$ and adding $b_{i+1}$, and so

$$
s_{i} T=\left(T^{(0)} \searrow T^{(1)} \searrow \cdots \searrow T^{(i-1)} \searrow s_{i} T^{(i)} \searrow T^{(i+1)} \searrow \cdots \searrow T^{(k)}\right)
$$

is the tableau constructed from $T$ by switching the order of adding the $i$-th and $(i+1)$-st boxes. Notice that if $c_{T}(i) \neq c_{T}(i+1) \pm 1$, then $s_{i} T$ is the only tableau that varies from $T$ only at the $i$-th position; otherwise, if $c_{T}(i)=c_{T}(i+1) \pm 1$, then there is no such tableau. 
Similarly, for any $\mu \in \mathscr{P}_{1}$, there are exactly one or two partitions $v \in \mathscr{P}$ that differ from $\mu$ by a box by Lemma 4.6. In other words, there are exactly one or two $v \in \mathscr{P}$ that could be the first step in a tableau with a given shifted content list $c_{T}(2), \ldots, c_{T}(k)$. Lemma 4.7 tells us that there is one when

$$
c_{T}(1)=\frac{1}{2}( \pm(a+p) \pm(b+q)),
$$

and there are two otherwise. So if $c_{T}(1) \neq \frac{1}{2}( \pm(a+p) \pm(b+q))$ define

$$
s_{0} T=\left(s_{0} T^{(0)} \searrow T^{(1)} \searrow \cdots \searrow T^{(k)}\right),
$$

where $s_{0} T^{(0)}$ is the unique partition built by moving $T^{(1)} / T^{(0)}$ to its complementary position (see Remark 4.3 or Figure 5). Since $c\left(T^{(1)} / s_{0} T^{(0)}\right)=a-p+b-q-$ $2 c\left(T^{(1)} / T^{(0)}\right)$, we have

$$
c_{s_{0} T}(1)=-c_{T}(1) \text {. }
$$

Proposition 5.2. Fix $\lambda \in \mathscr{P}_{k}$ and define

$$
\mathscr{H}^{\lambda}=\operatorname{span}_{\mathbb{C}}\left\{v_{T} \mid T \in \mathscr{T}_{\lambda}\right\}
$$

as a vector space with basis indexed by all tableaux from any $\mu \in \mathscr{P}$ to $\lambda$. Define an action of $\mathscr{H}_{k}^{\text {ext }}$ by

$$
\begin{aligned}
w_{i} \cdot v_{T} & =c_{T}(i) v_{T} & \text { for } 0 \leq i \leq k \\
t_{s_{i}} \cdot v_{T} & =\left[t_{i}\right]_{T, T} v_{T}+\left[t_{i}\right]_{T, s_{i} T} v_{s_{i} T} & \text { for } 1 \leq i \leq k-1 \\
x_{1} \cdot v_{T} & =\left[x_{1}\right]_{T, T} v_{T}+\left[x_{1}\right]_{T, s_{0} T} v_{s_{0} T}, &
\end{aligned}
$$

where $\left[t_{i}\right]_{T, s_{i} T}=0$ if and only if $c_{T}(i)=c_{T}(i+1) \pm 1$, and $\left[x_{1}\right]_{T, s_{0} T}=0$ if and only if $c_{T}(1)=\frac{1}{2}( \pm(a+p) \pm(b+q))$. Then $\mathscr{H}^{\lambda}$ is a simple $\mathscr{H}_{k}^{\text {ext }}$-module with respect to this action if and only if

(1) $\left[t_{i}\right]_{T, T}=1 /\left(c_{T}(i+1)-c_{T}(i)\right)$,

(2) $\left[x_{1}\right]_{T, T}=\frac{(a-p) c_{T}(1)+c_{T}^{2}(1)+\frac{1}{4}\left((a+p)^{2}-(b+q)^{2}\right)}{2 c_{T}(1)}$,

(3) (commutation)

$$
\begin{aligned}
& {\left[t_{i}\right]_{s_{j} T, s_{i} s_{j} T}\left[t_{j}\right]_{T, s_{j} T}=\left[t_{i}\right]_{T, s_{i} T}\left[t_{j}\right]_{s_{i} T, s_{j} s_{i} T} \quad \text { for } j \neq i \pm 1,} \\
& {\left[t_{i}\right]_{s_{0} T, s_{i} s_{0} T}\left[x_{1}\right]_{T, s_{0} T}=\left[t_{i}\right]_{T, s_{i} T}\left[x_{1}\right]_{s_{i} T, s_{0} s_{i} T} \text { for } i>1 \text {, }}
\end{aligned}
$$

(4) (involutions)

$$
\left[t_{i}\right]_{T, s_{i} T}\left[t_{i}\right]_{s_{i} T, T}=1-\left(\left[t_{i}\right]_{T, T}\right)^{2}
$$


(5) (quadratic relation)

$\left[x_{1}\right]_{T, s_{0} T}\left[x_{1}\right]_{s_{0} T, T}=-\frac{1}{\left(2 c_{T}(1)\right)^{2}}\left(c_{T}(1)+\frac{(a+p)+(b+q)}{2}\right)\left(c_{T}(1)-\frac{(a+p)-(b+q)}{2}\right)$
$\cdot\left(c_{T}(1)-\frac{(a+p)+(b+q)}{2}\right)\left(c_{T}(1)+\frac{(a+p)-(b+q)}{2}\right)$,

(6) (braid relations)

$\left[t_{i}\right]_{T, s_{i} T}\left[t_{i+1}\right]_{s_{i} T, s_{i+1} s_{i} T}\left[t_{i}\right]_{s_{i+1} s_{i} T, s_{i} s_{i+1} s_{i} T}$

$$
=\left[t_{i+1}\right]_{T, s_{i+1} T}\left[t_{i}\right]_{s_{i+1} T, s_{i} s_{i+1} T}\left[t_{i+1}\right]_{s_{i} s_{i+1} T, s_{i} s_{i+1} s_{i} T},
$$

$\left[x_{1}\right]_{s_{1} T, s_{0} s_{1} T}\left[x_{1}\right]_{s_{1} s_{0} s_{1} T, s_{0} s_{1} s_{0} s_{1} T}\left[t_{1}\right]_{T, s_{1} T}\left[t_{1}\right]_{s_{0} s_{1} T, s_{1} s_{0} s_{1} T}$

$$
=\left[x_{1}\right]_{T, s_{0} T}\left[x_{1}\right]_{s_{1} s_{0} T, s_{0} s_{1} s_{0} T}\left[t_{1}\right]_{s_{0} T, s_{1} s_{0} T}\left[t_{1}\right]_{s_{0} s_{1} s_{0} T, s_{1} s_{0} s_{1} s_{0} T} .
$$

Before we provide a proof of this proposition, we will give a nice example of such a seminormal representation.

Theorem 5.3. Define an action of $\mathscr{H}_{k}^{\text {ext }}$ on $\mathscr{H}^{\lambda}$ by

$$
\begin{aligned}
w_{i} \cdot v_{T} & =c_{T}(i) v_{T} & \text { for } 0 \leq i \leq k \\
t_{s_{i}} \cdot v_{T} & =\left[t_{i}\right]_{T, T} v_{T}+\left[t_{i}\right]_{T, s_{i} T} v_{s_{i} T} & \text { for } 1 \leq i \leq k-1, \\
x_{1} \cdot v_{T} & =\left[x_{1}\right]_{T, T} v_{T}+\left[x_{1}\right]_{T, s_{0} T} v_{s_{0} T}, &
\end{aligned}
$$

and

$$
\begin{gathered}
{\left[t_{i}\right]_{T, S}= \begin{cases}\sqrt{1-\left[t_{i}\right]_{T, T}^{2}} & \text { if } S \neq T, \\
1 /\left(c_{T}(i+1)-c_{T}(i)\right) & \text { if } S=T,\end{cases} } \\
{\left[x_{1}\right]_{T, S}= \begin{cases}\sqrt{\frac{-\frac{1}{\left(2 c_{T}(1)\right)^{2}}\left(c_{T}(1)+\frac{(a+p)+(b+q)}{2}\right)\left(c_{T}(1)-\frac{(a+p)-(b+q)}{2}\right)}{\cdot\left(c_{T}(1)-\frac{(a+p)+(b+q)}{2}\right)\left(c_{T}(1)+\frac{(a+p)-(b+q)}{2}\right)}} & \text { if } S \neq T, \\
\frac{(a-p) c_{T}(1)+c_{T}^{2}(1)+\frac{1}{4}\left((a+p)^{2}-(b+q)^{2}\right)}{2 c_{T}(1)} & \text { if } S=T .\end{cases} }
\end{gathered}
$$

With this action, $\mathscr{H}^{\lambda}$ is a simple $\mathscr{H}_{k}^{\mathrm{ext}}$-module.

Proof. The values for $\left[t_{i}\right]_{T, T}$ and $\left[x_{1}\right]_{T, T}$ are pulled directly from Proposition 5.2, so we need only check criteria 3-6: commutation, quadratic relation, and braid relations. We will verify these using the fact that $\left[x_{1}\right]_{T, S}$ and $\left[t_{i}\right]_{T, S}$ for $S \neq T$ are functions of shifted contents $c_{T}(j)$.

Commutation: For $j \neq i \pm 1, c_{T}(i)=c_{s_{j} T}(i), c_{T}(i+1)=c_{s_{j} T}(i+1), c_{T}(j)=$ $c_{s_{i} T}(j)$, and $c_{T}(j+1)=c_{s_{i} T}(j+1)$, so

$$
\left[t_{i}\right]_{s_{j} T, s_{i} s_{j} T}=\left[t_{i}\right]_{T, s_{i} T} \text { and }\left[t_{j}\right]_{T, s_{j} T}=\left[t_{j}\right]_{s_{i} T, s_{j} s_{i} T} .
$$

Similarly, for $i>1, c_{T}(i)=c_{s_{0} T}(i)$ and $c_{T}(i+1)=c_{s_{0} T}(i+1)$, so $\left[t_{i}\right]_{s_{0} T, s_{i} s_{0} T}=$ $\left[t_{i}\right]_{T, s_{i} T}$, and $c_{T}(1)=c_{s_{i} T}(1)$, so $\left[x_{1}\right]_{T, s_{0} T}=\left[x_{1}\right]_{s_{i} T, s_{0} s_{i} T}$. Thus criterion 3 is satisfied. 


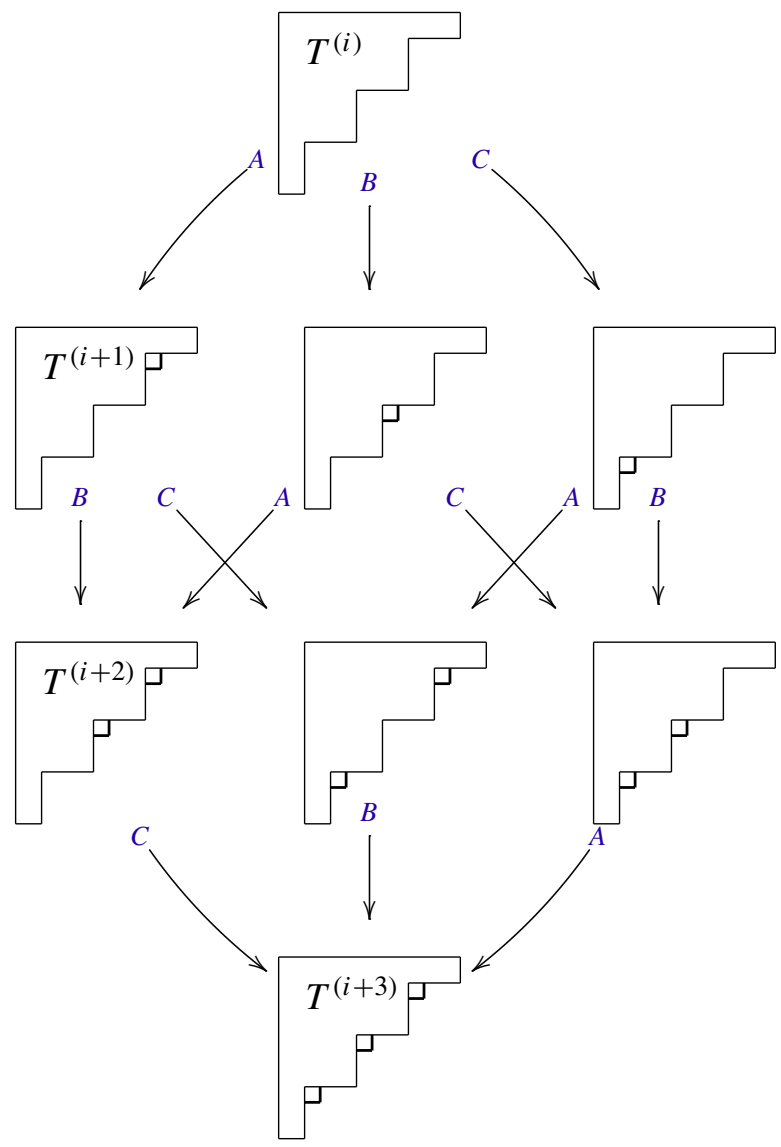

Figure 7. A generic subgraph of the Bratteli diagram in levels $i+1$ through $i+4$.

Quadratic relation: By (5-4), $\left[x_{1}\right]_{T, s_{0} T}=\left[x_{1}\right]_{T, s_{0} T}$, so criterion 4 is satisfied.

Braid relations: For the first braid relation, let $A=c_{T}(i), B=c_{T}(i+1)$, and $C=c_{T}(i+2)$. Either both sides of the equality

$\left[t_{i}\right]_{T, s_{i} T}\left[t_{i+1}\right]_{s_{i} T, s_{i+1} s_{i} T}\left[t_{i}\right]_{s_{i+1} s_{i} T, s_{i} s_{i+1} s_{i} T}$

$$
=\left[t_{i+1}\right]_{T, s_{i+1} T}\left[t_{i}\right]_{s_{i+1} T, s_{i} s_{i+1} T}\left[t_{i+1}\right]_{s_{i} s_{i+1} T, s_{i} s_{i+1} s_{i} T}
$$

are zero, or the six tableaux involved sit in a subgraph of the Bratteli diagram depicted in Figure 7. This encodes the fact that for whichever of these $S$ exist, their shifted contents follow the pattern in (5-5), and one can use these values to 


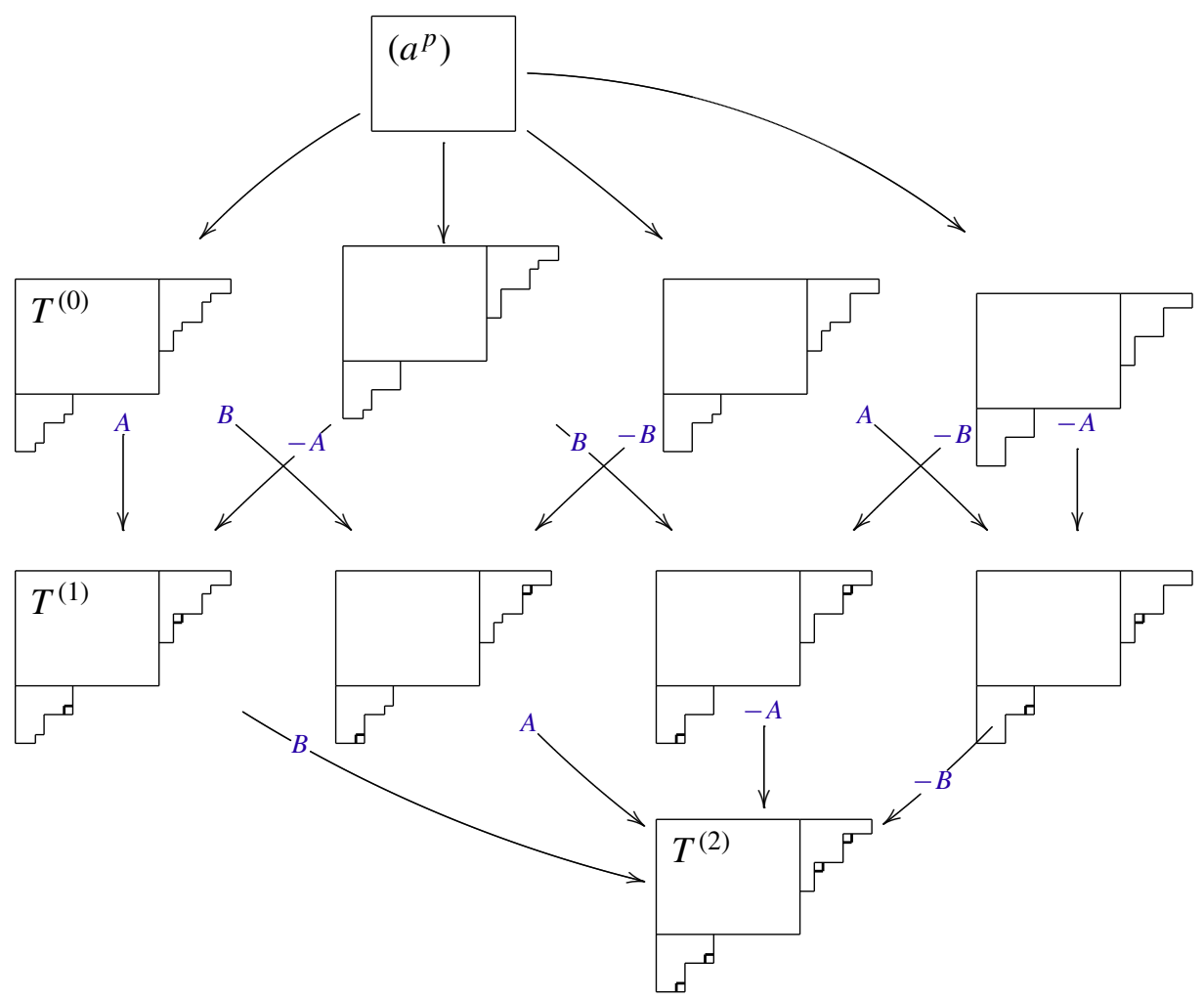

Figure 8. A generic subgraph of the Bratteli diagram in levels 0 through 3 .

check that the first braid relation is satisfied.

\begin{tabular}{c|cccccc}
$S \rightarrow$ & $T$ & $s_{i} T$ & $s_{i+1} T$ & $s_{i} s_{i+1} T$ & $s_{i+1} s_{i} T$ & $s_{i} s_{i+1} s_{i} T$ \\
\hline$c_{S}(i)$ & $A$ & $B$ & $A$ & $C$ & $B$ & $C$ \\
$c_{S}(i+1)$ & $B$ & $A$ & $C$ & $A$ & $C$ & $B$ \\
$c_{S}(i+2)$ & $C$ & $C$ & $B$ & $B$ & $A$ & $A$
\end{tabular}

For the second braid relation, let $A=c_{T}(1)$ and $B=c_{T}(2)$. So either both sides of the equality

$\left[x_{1}\right]_{s_{1} T, s_{0} s_{1} T}\left[x_{1}\right]_{s_{1} s_{0} s_{1} T, s_{0} s_{1} s_{0} s_{1} T}\left[t_{1}\right]_{T, s_{1} T}\left[t_{1}\right]_{s_{0} s_{1} T, s_{1} s_{0} s_{1} T}$

$$
=\left[x_{1}\right]_{T, s_{0} T}\left[x_{1}\right]_{s_{1} s_{0} T, s_{0} s_{1} s_{0} T}\left[t_{1}\right]_{s_{0} T, s_{1} s_{0} T}\left[t_{1}\right]_{s_{0} s_{1} s_{0} T, s_{1} s_{0} s_{1} s_{0} T}
$$

are zero, or the eight tableaux involved sit in a subgraph of the Bratteli diagram depicted in Figure 8. This encodes the fact that for whichever of these $S$ exist, their shifted contents follow the pattern in (5-6), and one can use these values to check 
that the first braid relation is satisfied

\begin{tabular}{c|rrrrrrrr}
$S \rightarrow$ & $T$ & $s_{0} T$ & $s_{1} T$ & $s_{0} s_{1} T$ & $s_{1} s_{0} T$ & $s_{0} s_{1} s_{0} T$ & $s_{1} s_{0} s_{1} T$ & $s_{0} s_{1} s_{0} s_{1} T$ \\
\hline$c_{S}(1)$ & $A$ & $-A$ & $B$ & $-B$ & $B$ & $-B$ & $A$ & $-A$ \\
$c_{S}(2)$ & $B$ & $B$ & $A$ & $A$ & $-A$ & $-A$ & $-B$ & $-B$
\end{tabular}

Thus criterion 6 is satisfied, concluding the proof of Theorem 5.3.

Proof of Proposition 5.2. The proof comes in two parts. In the first, we check that the relations in the presentation of $\mathscr{H}_{k}^{\text {ext }}$ given in Theorem 3.2 hold, showing that $\mathscr{H}^{\lambda}$ is a $\mathscr{H}_{k}^{\text {ext }}$-module. In the second, we verify that $\mathscr{H}^{\lambda}$ is simple.

Part 1: $\mathscr{H}^{\lambda}$ is $\boldsymbol{a} \mathscr{H}_{\boldsymbol{k}}^{\text {ext }}$-module. By (3-9), the elements $w_{0}, w_{1}, \ldots, w_{k}$ generate a commutative subalgebra of $\mathcal{H}_{k}^{\text {ext }}$, so we begin by fixing the diagonal action as stated above,

$$
\begin{aligned}
& w_{0} v_{T}=\left(a b q+2 \sum_{B \in \mathscr{B}_{\lambda}}\left(c(B)-\frac{1}{2}(a-p+b-q)\right)\right) v_{T}, \\
& w_{i} v_{T}=c_{T}(i) v_{T} \quad \text { for } 1 \leq i \leq k .
\end{aligned}
$$

Now write

$$
t_{s_{i}} v_{T}=\sum_{S \in \mathcal{T}_{\lambda}}\left[t_{i}\right]_{T, S} v_{S} \quad \text { and } \quad x_{1} v_{T}=\sum_{S \in \mathcal{T}_{\lambda}}\left[x_{1}\right]_{T, S} v_{S}
$$

where $\mathscr{T}_{\lambda}$ is the set of tableaux $(5-1)$ and $\left[t_{i}\right]_{T, S},\left[x_{1}\right]_{T, S} \in \mathbb{C}$.

Claim a. Relations $t_{s_{i}}^{2}=1,(3-6)$, and (3-10) are satisfied if and only if

$$
\begin{aligned}
t_{s_{i}} v_{T} & =\left[t_{i}\right]_{T, T} v_{T}+\left[t_{i}\right]_{T, s_{i} T} v_{s_{i} T} \quad \text { for } i=1, \ldots, k-1, \\
{\left[t_{i}\right]_{T, T} } & =\frac{1}{c_{T}(i+1)-c_{T}(i)} \quad \text { and } \quad\left[t_{i}\right]_{T, S}\left[t_{i}\right]_{S, T}=1-\left(\left[t_{i}\right]_{T, T}\right)^{2} .
\end{aligned}
$$

Proof. The first commutation relation (3-6), $t_{s_{i}} w_{j}=w_{j} t_{s_{i}}$ for $j \neq i, i+1$, implies

$$
t_{s_{i}} w_{j} v_{T}=\sum_{S \in \mathcal{T}_{\lambda}} c_{T}(j)\left[t_{i}\right]_{T, S} v_{S}=w_{j} t_{S_{i}} v_{T}=\sum_{S \in \mathcal{T}_{\lambda}} c_{S}(j)\left[t_{i}\right]_{T, S} v_{S}
$$

So for each $S$, either

$$
\left[t_{i}\right]_{T, S}=0 \quad \text { or } \quad c_{T}(j)=c_{S}(j) \quad \text { for all } j \neq i, i+1 .
$$

The first twisting relation (3-10), together with relation $t_{s_{i}}^{2}=1$, requires

$$
t_{s_{i}} w_{i}-w_{i+1} t_{s_{i}}=-1=w_{i} t_{s_{i}}-t_{s_{i}} w_{i+1},
$$


that is,

$$
\begin{aligned}
\left(t_{s_{i}} w_{i}-w_{i+1} t_{s_{i}}\right) v_{T} & =\sum_{S \in \mathcal{T}_{\lambda}}\left(c_{T}(i)-c_{S}(i+1)\right)\left[t_{i}\right]_{T, S} v_{S}=-v_{T} \\
& =\left(w_{i} t_{S_{i}}-t_{s_{i}} w_{i+1}\right) v_{T}=\sum_{S \in \mathcal{T}_{\lambda}}\left(c_{S}(i)-c_{T}(i+1)\right)\left[t_{i}\right]_{T, S} v_{S}
\end{aligned}
$$

So

$$
\left[t_{i}\right]_{T, T}=\frac{1}{c_{T}(i+1)-c_{T}(i)}
$$

and for $S \neq T$, either

$$
c_{S}(i+1)=c_{T}(i) \quad \text { and } \quad c_{S}(i)=c_{T}(i+1) \quad \text { or } \quad\left[t_{i}\right]_{T, S}=0 .
$$

By Lemma 5.1, Equations (5-7) and (5-9) tell us

$$
t_{s_{i}} v_{T}=\left[t_{i}\right]_{T, T} v_{T}+\left[t_{i}\right]_{T, s_{i} T} v_{s_{i} T} \text { for } i=1, \ldots, k-1,
$$

where $\left[t_{i}\right]_{T, s_{i} T}=0$ if $c_{T}(i)=c_{T}(i+1) \pm 1$. Finally, the involution relation $t_{s_{i}}^{2}=1$ implies

$$
\left[t_{i}\right]_{T, T}=-\left[t_{i}\right]_{s_{i} T, s_{i} T} \text { and } \quad\left[t_{i}\right]_{T, S}\left[t_{i}\right]_{S, T}=1-\left(\left[t_{i}\right]_{T, T}\right)^{2} .
$$

The first is implied by $\left[t_{i}\right]_{T, T}=(1) / c_{T}(i+1)-c_{T}(i)$, but the second places a new condition on coefficients.

Claim b. Relation (3-7) is satisfied if and only if

$$
x_{1} v_{T}=\left[x_{1}\right]_{T, T} v_{T}+\left[x_{1}\right]_{T, s_{0} T} v_{s_{0} T},
$$

where $\left[x_{1}\right]_{T, s_{0} T}=0$ if $c_{T}(1)= \pm(a+p) \pm(b+q)$. Furthermore, (3-5), (3-11), and (3-12) are additionally satisfied if and only if

$$
\left[x_{1}\right]_{T, T}=\frac{(a-p) c_{T}(1)+c_{T}^{2}(1)+\frac{1}{4}\left((a+p)^{2}-(b+q)^{2}\right)}{2 c_{T}(1)}
$$

and

$$
\begin{array}{r}
{\left[x_{1}\right]_{T, s_{0} T}\left[x_{1}\right]_{s_{0} T, T}=-\frac{1}{\left(2 c_{T}(1)\right)^{2}}\left(c_{T}(1)+\frac{(a+p)+(b+q)}{2}\right)\left(c_{T}(1)-\frac{(a+p)-(b+q)}{2}\right)} \\
\cdot\left(c_{T}(1)-\frac{(a+p)+(b+q)}{2}\right)\left(c_{T}(1)+\frac{(a+p)-(b+q)}{2}\right) .
\end{array}
$$

Proof. The relation $x_{1} w_{i}=w_{i} x_{1}$ for $i>1$ implies

$$
x_{1} w_{i} v_{T}=\sum_{S \in T_{\lambda}} c_{T}(i)\left[x_{1}\right]_{T, S} v_{S}=w_{i} x_{1} v_{T}=\sum_{S \in T_{\lambda}} c_{S}(i)\left[x_{1}\right]_{T, S} v_{S} .
$$


So by Lemmas 4.6 and 5.1,

$$
x_{1} v_{T}=\left[x_{1}\right]_{T, T} v_{T}+\left[x_{1}\right]_{T, s_{0} T} v_{s_{0} T}
$$

where $\left[x_{1}\right]_{T, s_{0} T}=0$ if $c_{T}(1)= \pm(a+p) \pm(b+q)$.

Now let $K=\frac{1}{4}\left((a+p)^{2}-(b+q)^{2}\right)$, so the third twisting relation (3-12),

$$
x_{1} w_{1}=-w_{1} x_{1}+(a-p) w_{1}+w_{1}^{2}+K,
$$

says

$$
\begin{aligned}
\left(x_{1} w_{1}+w_{1} x_{1}\right) v_{T} & =\left(c_{T}(1)+c_{T}(1)\right)\left[x_{1}\right]_{T, T} v_{T}+\left(c_{T}(1)+c_{s_{0} T}(1)\right)\left[x_{1}\right]_{T, s_{0} T} v_{s_{0} T} \\
& =2 c_{T}(1)\left[x_{1}\right]_{T, T} v_{T}=\left((a-p) w_{1}+w_{1}^{2}+K\right) v_{T} \\
& =\left((a-p) c_{T}(1)+\left(c_{T}(1)\right)^{2}+K\right) v_{T}
\end{aligned}
$$

So

$$
\left[x_{1}\right]_{T, T}=\frac{\left((a-p) c_{T}(1)+\left(c_{T}(1)\right)^{2}+K\right)}{2 c_{T}(1)} .
$$

If $S=s_{0} T$ exists, then the quadratic relation (3-5) implies

$$
\begin{aligned}
x_{1}^{2} v_{T} & =\left(\left[x_{1}\right]_{T, T}^{2}+\left[x_{1}\right]_{T, S}\left[x_{1}\right]_{S, T}\right) v_{T}+\left(\left[x_{1}\right]_{T, T}\left[x_{1}\right]_{T, S}+\left[x_{1}\right]_{T, S}\left[x_{1}\right]_{S, S}\right) v_{S} \\
& =(a-p) x_{1}+a p=\left((a-p)\left[x_{1}\right]_{T, T}+a p\right) v_{T}+(a-p)\left[x_{1}\right]_{T, S} v_{S} .
\end{aligned}
$$

We could conclude $\left(\left[x_{1}\right]_{T, T}\left[x_{1}\right]_{T, S}+\left[x_{1}\right]_{T, S}\left[x_{1}\right]_{S, S}\right)=(a-p)\left[x_{1}\right]_{T, S}$ from (5-11), so this simply tells us that

$$
\begin{aligned}
{\left[x_{1}\right]_{T, S}\left[x_{1}\right]_{S, T}=} & -\left[x_{1}\right]_{T, T}^{2}+(a-p)\left[x_{1}\right]_{T, T}+a p \\
= & -\left(\frac{\left((a-p) c_{T}(1)+c_{T}^{2}(1)+K\right)}{2 c_{T}(1)}\right)^{2} \\
+(a-p)\left(\frac{\left((a-p) c_{T}(1)+c_{T}^{2}(1)+K\right)}{2 c_{T}(1)}\right)+a p & \\
= & -\frac{1}{4 c_{T}^{2}(1)}\left(c_{T}(1)+\frac{(a+p)+(b+q)}{2}\right)\left(c_{T}(1)-\frac{(a+p)-(b+q)}{2}\right) \\
& \cdot\left(c_{T}(1)-\frac{(a+p)+(b+q)}{2}\right)\left(c_{T}(1)+\frac{(a+p)-(b+q)}{2}\right) .
\end{aligned}
$$

Finally, the second twisting relation (3-11) implies

$$
\begin{aligned}
x_{1}\left(w_{0}+w_{1}\right) v_{T} & =\left(c_{T}(0)+c_{T}(1)\right)\left[x_{1}\right]_{T, T} v_{T}+\left(c_{T}(0)+c_{T}(1)\right)\left[x_{1}\right]_{T, s_{0} T} v_{s_{0} T} \\
& =\left(w_{0}+w_{1}\right) x_{1} v_{T} \\
& =\left(c_{T}(0)+c_{T}(1)\right)\left[x_{1}\right]_{T, T} v_{T}+\left(c_{s_{0} T}(0)+c_{s_{0} T}(1)\right)\left[x_{1}\right]_{T, s_{0} T} v_{s_{0} T} .
\end{aligned}
$$

So we require

$$
\left[x_{1}\right]_{T, s_{0} T}=\left[x_{1}\right]_{T, s_{0} T}=0 \quad \text { or } \quad c_{T}(0)+c_{T}(1)=c_{s_{0} T}(0)+c_{s_{0} T}(1) .
$$


Recall from (5-4) that if $v_{s_{0} T}$ exists, then $c_{s_{0} T}(1)=-c_{T}(1)$. So this requirement is equivalent to

$$
\left[x_{1}\right]_{T, s_{0} T}=0 \quad \text { or } \quad c_{T}(1)=\frac{1}{2}\left(c_{s_{0} T}(0)-c_{T}(0)\right),
$$

and is therefore a consequence of the construction in Lemmas 4.4 and 4.5.

Claim c. The second relation in (3-3) and relation (3-8) are satisfied if and only if

$$
\begin{aligned}
{\left[t_{i}\right]_{s_{j} T, s_{i} s_{j} T}\left[t_{j}\right]_{T, s_{j} T}=\left[t_{i}\right]_{T, s_{i} T}\left[t_{j}\right]_{s_{i} T, s_{j} s_{i} T} } & \text { for } j \neq i \pm 1, \\
{\left[t_{i}\right]_{s_{0} T, s_{i} s_{0} T}\left[x_{1}\right]_{T, s_{0} T}=\left[t_{i}\right]_{T, s_{i} T}\left[x_{1}\right]_{s_{i} T, s_{0} s_{i} T} } & \text { for } i>1,
\end{aligned}
$$

respectively.

Proof. For $j \neq i \pm 1$, the second relation in (3-3) implies

$$
\begin{aligned}
& t_{s_{i}} t_{s_{j}} v_{T}=\left[t_{i}\right]_{T, T}\left[t_{j}\right]_{T, T} v_{T}+\left[t_{i}\right]_{T, s_{i} T}\left[t_{j}\right]_{T, T} v_{s_{i} T} \\
& \quad+\left[t_{i}\right]_{s_{j} T, s_{j} T}\left[t_{j}\right]_{T, s_{j} T} v_{s_{j} T}+\left[t_{i}\right]_{s_{j} T, s_{i} s_{j} T}\left[t_{j}\right]_{T, s_{j} T} v_{s_{i} s_{j} T} \\
& =t_{s_{j}} t_{s_{i}} v_{T}=\left[t_{i}\right]_{T, T}\left[t_{j}\right]_{T, T} v_{T}+\left[t_{i}\right]_{T, s_{i} T}\left[t_{j}\right]_{s_{i} T, s_{i} T} v_{s_{i} T} \\
& \quad+\left[t_{i}\right]_{T, T}\left[t_{j}\right]_{T, s_{i} T} v_{s_{j} T}+\left[t_{i}\right]_{T, s_{i} T}\left[t_{j}\right]_{s_{i} T, s_{j} s_{i} T} v_{s_{j} s_{i} T} .
\end{aligned}
$$

If $s_{i} T$ and $s_{j} T$ exist, we already know $\left[t_{j}\right]_{T, T}=\left[t_{j}\right]_{s_{i} T, s_{i} T}$ and $\left[t_{i}\right]_{s_{j} T, s_{j} T}=\left[t_{i}\right]_{T, T}$ because $c_{T}(j)=c_{s_{i} T}(j)$ and $c_{T}(i)=c_{s_{j} T}(i)$ for $j \neq i \pm 1$. However, since $s_{i} s_{j} T=$ $s_{j} s_{i} T$, we gain the requirement

$$
\left[t_{i}\right]_{s_{j} T, s_{i} s_{j} T}\left[t_{j}\right]_{T, s_{j} T}=\left[t_{i}\right]_{T, s_{i} T}\left[t_{j}\right]_{s_{i} T, s_{j} s_{i} T} .
$$

Similarly, for $i>1$, relation (3-8) implies

$$
\begin{aligned}
t_{s_{i}} x_{1} v_{T}=\left[t_{i}\right]_{T, T}\left[x_{1}\right]_{T, T} v_{T}+\left[t_{i}\right]_{s_{0} T, s_{0} T}\left[x_{1}\right]_{T, s_{0} T} v_{s_{0} T} & \\
& +\left[t_{i}\right]_{T, s_{i} T}\left[x_{1}\right]_{T, T} v_{s_{i} T}+\left[t_{i}\right]_{s_{0} T, s_{i} s_{0} T}\left[x_{1}\right]_{T, s_{0} T} v_{s_{i} s_{0} T} \\
=x_{1} t_{s_{i}} v_{T}=\left[t_{i}\right]_{T, T}\left[x_{1}\right]_{T, T} v_{T}+\left[t_{i}\right]_{T, T}\left[x_{1}\right]_{T, s_{0} T} v_{s_{0} T} & +\left[t_{i}\right]_{T, s_{i} T}\left[x_{1}\right]_{s_{i} T, s_{i} T} v_{s_{i} T}+\left[t_{i}\right]_{T, s_{i} T}\left[x_{1}\right]_{s_{i} T, s_{0} s_{i} T} v_{s_{i} s_{0} T}
\end{aligned}
$$

since $s_{0} s_{i} T=s_{i} s_{0} T$ for $i>1$. If $s_{0} T$ and $s_{i} T$ exist, we already require that

$$
\left[t_{i}\right]_{s_{0} T, s_{0} T}=\left[t_{i}\right]_{T, T} \text { and }\left[x_{1}\right]_{T, T}=\left[x_{1}\right]_{s_{i} T, s_{i} T},
$$

since $c_{T}(i)=c_{s_{0} T}(i), c_{T}(i+1)=c_{s_{0} T}(i+1)$, and $c_{T}(1)=c_{s_{i} T}(1)$. However, given $s_{0} T, s_{i} T$, and $s_{0} s_{i} T$ exist, we gain the requirement

$$
\left[t_{i}\right]_{s_{0} T, s_{i} s_{0} T}\left[x_{1}\right]_{T, s_{0} T}=\left[t_{i}\right]_{T, s_{i} T}\left[x_{1}\right]_{s_{i} T, s_{0} s_{i} T} .
$$

Claim d. The braid relation (3-3) is satisfied if and only if $\left[t_{i}\right]_{T, s_{i} T}\left[t_{i+1}\right]_{s_{i} T, s_{i+1} s_{i} T}\left[t_{i}\right]_{s_{i+1} s_{i} T, s_{i} s_{i+1} s_{i} T}$

$$
=\left[t_{i+1}\right]_{T, s_{i+1} T}\left[t_{i}\right]_{s_{i+1} T, s_{i} s_{i+1} T}\left[t_{i+1}\right]_{s_{i} s_{i+1} T, s_{i} s_{i+1} s_{i} T} .
$$


Proof. If $v_{S}$ exists for $S=s_{i} T, s_{i+1} T, s_{i} s_{i+1} T, s_{i+1} s_{i} T, s_{i} s_{i+1} s_{i} T$, then

$$
\begin{aligned}
t_{s_{i}} t_{s_{i+1}} t_{s_{i}} v_{T} t_{s_{i}} t_{s_{i+1}}\left(\left[t_{i}\right]_{T, T} v_{T}+\left[t_{i}\right]_{T, s_{i} T} v_{s_{i} T}\right) \\
=\left(\left[t_{i}\right]_{T, T}^{2}\left[t_{i+1}\right]_{T, T}+\left[t_{i}\right]_{T, s_{i} T}\left[t_{i+1}\right]_{s_{i} T, s_{i} T}\left[t_{i}\right]_{s_{i} T, T}\right) v_{T} \\
\quad+\left(\left[t_{i}\right]_{T, T}\left[t_{i+1}\right]_{T, T}\left[t_{i}\right]_{T, s_{i} T}+\left[t_{i}\right]_{T, s_{i} T}\left[t_{i+1}\right]_{s_{i} T, s_{i} T}\left[t_{i}\right]_{s_{i} T, s_{i} T}\right) v_{s_{i} T} \\
\quad+\left(\left[t_{i}\right]_{T, T}\left[t_{i+1}\right]_{T, s_{i+1} T}\left[t_{i}\right]_{s_{i+1} T, s_{i+1} T}\right) v_{s_{i+1} T} \\
\quad+\left(\left[t_{i}\right]_{T, T}\left[t_{i+1}\right]_{T, s_{i+1} T}\left[t_{i}\right]_{s_{i+1} T, s_{i} s_{i+1} T}\right) v_{s_{i} s_{i+1} T} \\
\quad+\left(\left[t_{i}\right]_{T, s_{i} T}\left[t_{i+1}\right]_{s_{i} T, s_{i+1} s_{i} T}\left[t_{i}\right]_{s_{i+1} s_{i} T, s_{i+1} s_{i} T}\right) v_{s_{i+1} s_{i} T} \\
\quad+\left(\left[t_{i}\right]_{T, s_{i} T}\left[t_{i+1}\right]_{s_{i} T, s_{i+1} s_{i} T}\left[t_{i}\right]_{s_{i+1} s_{i} T, s_{i} s_{i+1} s_{i} T}\right) v_{s_{i} s_{i+1} s_{i} T}
\end{aligned}
$$

because $s_{i} s_{i} T=T$. Similarly,

$$
\begin{aligned}
t_{s_{i+1}} t_{s_{i}} t_{s_{i+1}} v_{T}=( & {\left.\left[t_{i+1}\right]_{T, T}^{2}\left[t_{i}\right]_{T, T}+\left[t_{i+1}\right]_{T, s_{i+1} T}\left[t_{i}\right]_{s_{i+1} T, s_{i+1} T}\left[t_{i+1}\right]_{s_{i+1} T, T}\right) v_{T} } \\
& +\left(\left[t_{i+1}\right]_{T, T}\left[t_{i}\right]_{T, T}\left[t_{i+1}\right]_{T, s_{i+1} T}\right. \\
& \left.\quad+\left[t_{i+1}\right]_{T, s_{i+1} T}\left[t_{i}\right]_{s_{i+1} T, s_{i+1} T}\left[t_{i+1}\right]_{s_{i+1} T, s_{i+1} T}\right) v_{s_{i+1}} T \\
& +\left[t_{i+1}\right]_{T, T}\left[t_{i}\right]_{T, s_{i} T}\left[t_{i+1}\right]_{s_{i} T, s_{i} T} v_{s_{i} T} \\
& +\left[t_{i+1}\right]_{T, T}\left[t_{i}\right]_{T, s_{i} T}\left[t_{i+1}\right]_{s_{i} T, s_{i+1} s_{i} T} v_{s_{i+1} s_{i} T} \\
& +\left[t_{i+1}\right]_{T, s_{i+1} T}\left[t_{i}\right]_{s_{i+1} T, s_{i} s_{i+1} T}\left[t_{i+1}\right]_{s_{i} s_{i+1} T, s_{i} s_{i+1} T} v_{s_{i} s_{i+1} T} \\
& +\left[t_{i+1}\right]_{T, s_{i+1} T}\left[t_{i}\right]_{s_{i+1} T, s_{i} s_{i+1} T}\left[t_{i+1}\right]_{s_{i} s_{i+1} T, s_{i} s_{i+1} s_{i} T} v_{s_{i} s_{i+1} s_{i} T .}
\end{aligned}
$$

To check the identity $t_{s_{i}} t_{s_{i+1}} t_{s_{i}} v_{T}=t_{s_{i+1}} t_{s_{i}} t_{s_{i+1}} v_{T}$, we show that each coefficient in $t_{s_{i}} t_{s_{i+1}} t_{s_{i}} v_{T}-t_{s_{i+1}} t_{s_{i}} t_{s_{i+1}} v_{T}$ is 0 , noting that if some $S$ does not exist, the result is trivial.

Let $A=c_{T}(i), B=c_{T}(i+1)$, and $C=c_{T}(i+2)$. By definition, for whichever of these $S$ exist, their shifted contents are given by the table in (5-5). So, by using the condition that $\left[t_{i}\right]_{T, T}=1 /\left(c_{T}(i+1)-c_{T}(i)\right)$ to simplify the expansion above, we find that the coefficients vanish on each $v_{S}$, for $S=T, s_{i} T, s_{i+1} T, s_{i} s_{i+1} T$, $s_{i+1} s_{i} T$. The remaining term,

$\left(\left[t_{i}\right]_{T, s_{i} T}\left[t_{i+1}\right]_{s_{i} T, s_{i+1} s_{i} T}\left[t_{i}\right]_{s_{i+1} s_{i} T, s_{i} s_{i+1} s_{i} T}\right.$

$$
\left.-\left[t_{i+1}\right]_{T, s_{i+1} T}\left[t_{i}\right]_{s_{i+1} T, s_{i} s_{i+1} T}\left[t_{i+1}\right]_{s_{i} s_{i+1} T, s_{i} s_{i+1} s_{i} T}\right) v_{s_{i} s_{i+1} s_{i} T}
$$

cannot be reduced using the determined values, and so we add the assumption that this coefficient is 0 .

Claim e. The braid relation (3-4) is satisfied if and only if

$\left[x_{1}\right]_{s_{1} T, s_{0} s_{1} T}\left[x_{1}\right]_{s_{1} s_{0} s_{1} T, s_{0} s_{1} s_{0} s_{1} T}\left[t_{1}\right]_{T, s_{1} T}\left[t_{1}\right]_{s_{0} s_{1} T, s_{1} s_{0} s_{1} T}$

$$
=\left[x_{1}\right]_{T, s_{0} T}\left[x_{1}\right]_{s_{1} s_{0} T, s_{0} s_{1} s_{0} T}\left[t_{1}\right]_{s_{0} T, s_{1} s_{0} T}\left[t_{1}\right]_{s_{0} s_{1} s_{0} T, s_{1} s_{0} s_{1} s_{0} T} .
$$


Proof. Let $a_{T}=\left[x_{1}\right]_{T, T}, b_{T}=\left[x_{1}\right]_{T, s_{0} T}, d_{T}=\left[t_{1}\right]_{T, T}$ and $e_{T}=\left[t_{1}\right]_{T, s_{1} T}$. So

$$
\begin{aligned}
x_{1} t_{s_{1}} v_{T}= & a_{T} d_{T} v_{T}+b_{T} d_{T} v_{s_{0} T}+a_{s_{1} T} e_{T} v_{s_{1} T}+b_{s_{1} T} e_{T} v_{s_{0} s_{1} T}, \quad \text { and } \\
x_{1} t_{s_{1}} x_{1} t_{s_{1}} v_{T}= & \left(a_{T}^{2} d_{T}^{2}+b_{T} b_{s_{0} T} d_{T} d_{s_{0} T}+a_{T} a_{s_{1} T} e_{T} e_{s_{1} T}\right) v_{T} \\
& +\left(a_{T} b_{T} d_{T}^{2}+a_{s_{0} T} b_{T} d_{T} d_{s_{0} T}+a_{s_{1} T} b_{T} e_{T} e_{s_{1} T}\right) v_{s_{0} T} \\
& +\left(a_{T} a_{s_{1} T} d_{T} e_{T}+a_{s_{1} T}^{2} d_{s_{1} T} e_{T}+b_{s_{1} T} b_{s_{0} s_{1} T} d_{s_{0} s_{1} T} e_{T}\right) v_{s_{1} T} \\
& +\left(a_{T} b_{s_{1} T} d_{T} e_{T}+a_{s_{1} T} b_{s_{1} T} d_{s_{1} T} e_{T}+a_{s_{0} s_{1} T} b_{s_{1} T} e_{T} d_{s_{0} s_{1} T}\right) v_{s_{0} s_{1} T} \\
& +\left(a_{s_{1} s_{0} T} b_{T} d_{T} e_{s_{0} T}\right) v_{s_{1} s_{0} T}+\left(b_{s_{1} s_{0} T} b_{T} d_{T} e_{s_{0} T}\right) v_{s_{0} s_{1} s_{0} T} \\
& +\left(a_{s_{1} s_{0} s_{1} T} b_{s_{1} T} e_{T} e_{s_{0} s_{1} T}\right) v_{s_{1} s_{0} s_{1} T}+\left(b_{s_{1} T} b_{s_{1} s_{0} s_{1} T} e_{T} e_{s_{0} s_{1} T}\right) v_{s_{0} s_{1} s_{0} s_{1} T}
\end{aligned}
$$

and so

$$
\begin{aligned}
\left(x_{1} t_{s_{1}} x_{1} t_{s_{1}}\right. & \left.+x_{1} t_{s_{1}}\right) v_{T} \\
= & \left(a_{T}^{2} d_{T}^{2}+b_{T} b_{s_{0} T} d_{T} d_{s_{0} T}+a_{T} a_{s_{1} T} e_{T} e_{s_{1} T}+a_{T} d_{T}\right) v_{T} \\
& +\left(a_{T} b_{T} d_{T}^{2}+a_{s_{0} T} b_{T} d_{T} d_{s_{0} T}+a_{s_{1} T} b_{T} e_{T} e_{s_{1} T}+b_{T} d_{T}\right) v_{s_{0} T} \\
& +\left(a_{T} a_{s_{1} T} d_{T} e_{T}+a_{s_{1} T}^{2} d_{s_{1} T} e_{T}+b_{s_{1} T} b_{s_{0} s_{1} T} d_{s_{0} s_{1} T} e_{T}+a_{s_{1} T} e_{T}\right) v_{s_{1} T} \\
& +\left(a_{T} b_{s_{1} T} d_{T} e_{T}+a_{s_{1} T} b_{s_{1} T} d_{s_{1} T} e_{T}+a_{s_{0} s_{1} T} b_{s_{1} T} e_{T} d_{s_{0} s_{1} T}+b_{s_{1} T} e_{T}\right) v_{s_{0} s_{1} T} \\
& +\left(a_{s_{1} s_{0} T} b_{T} d_{T} e_{s_{0} T}\right) v_{s_{1} s_{0} T}+\left(b_{s_{1} s_{0} T} b_{T} d_{T} e_{s_{0} T}\right) v_{s_{0} s_{1} s_{0} T} \\
& +\left(a_{s_{1} s_{0} s_{1} T} b_{s_{1} T} e_{T} e_{s_{0} s_{1} T}\right) v_{s_{1} s_{0} s_{1} T}+\left(b_{s_{1} T} b_{s_{1} s_{0} s_{1} T} e_{T} e_{s_{0} s_{1} T}\right) v_{s_{0} s_{1} s_{0} s_{1} T}
\end{aligned}
$$

Similarly, since $s_{0} s_{1} s_{0} s_{1} T=s_{1} s_{0} s_{1} s_{0} T$,

$$
\begin{aligned}
\left(t_{s_{1}} x_{1} t_{s_{1}} x_{1}\right. & \left.+t_{s_{1}} x_{1}\right) v_{T} \\
= & \left(a_{T}^{2} d_{T}^{2}+b_{T} b_{s_{0} T} d_{T} d_{s_{0} T}+a_{T} a_{s_{1} T} e_{T} e_{s_{1} T}+a_{T} d_{T}\right) v_{T} \\
& +\left(a_{T} b_{T} d_{T} d_{s_{0} T}+a_{s_{0} T} b_{T} d_{s_{0} T}^{2}+a_{s_{1} s_{0} T} b_{T} e_{s_{0} T} e_{s_{1} s_{0} T}+b_{T} d_{s_{0} T}\right) v_{s_{0} T} \\
& +\left(a_{T}^{2} d_{T} e_{T}+b_{T} b_{s_{0} T} d_{s_{0} T} e_{T}+a_{T} a_{s_{1} T} d_{s_{1} T} e_{T}+a_{T} e_{T}\right) v_{s_{1} T} \\
& +\left(a_{T} b_{s_{1} T} d_{s_{0} s_{1} T} e_{T}\right) v_{s_{0} s_{1} T} \\
& +\left(a_{T} b_{T} d_{T} e_{s_{0} T}+a_{s_{0} T} b_{T} d_{s_{0} T} e_{s_{0} T}+a_{s_{1} s_{0} T} b_{T} d_{s_{1} s_{0} T} e_{s_{0} T}+b_{T} e_{s_{0} T}\right) v_{s_{1} s_{0} T} \\
& +\left(b_{T} b_{s_{1} s_{0} T} d_{s_{0} s_{1} s_{0} T} e_{s_{0} T}\right) v_{s_{0} s_{1} s_{0} T}+\left(a_{T} b_{s_{1} T} e_{T} e_{s_{0} s_{1} T}\right) v_{s_{1} s_{0} s_{1} T} \\
& +\left(b_{T} b_{s_{1} s_{0} T} e_{s_{0} T} e_{s_{0} s_{1} s_{0} T}\right) v_{s_{0} s_{1} s_{0} s_{1} T}
\end{aligned}
$$

Let $A=c_{T}$ (1) and $B=c_{T}$ (2). By definition, for whichever of these $S$ exist, their shifted contents are given by the table in (5-6). Thus the values of $a_{S}$ and $d_{S}$ 
are given by

\begin{tabular}{|c|c|c|c|c|}
\hline$S \rightarrow$ & $T$ & $s_{0} T$ & $s_{1} T$ & $s_{0} s_{1} T$ \\
\hline $\begin{array}{l}a_{S} \\
d_{S}\end{array}$ & $\begin{array}{l}a_{T} \\
\frac{1}{B-A}\end{array}$ & $\begin{array}{c}-a_{T}+(a-p) \\
\frac{1}{B+A}\end{array}$ & $\begin{array}{ll}a_{s_{1} T} & -a_{s_{1}} T \\
-d_{T} & \end{array}$ & $\begin{array}{l}+(a-p) \\
d_{s_{0} T}\end{array}$ \\
\hline$S \rightarrow$ & $s_{1} s_{0} T$ & $s_{0} s_{1} s_{0} T$ & $s_{1} s_{0} s_{1} T$ & $s_{0} s_{1} s_{0} s_{1} T$ \\
\hline $\begin{array}{l}a_{S} \\
d_{S}\end{array}$ & $\begin{array}{l}a_{s_{1} T} \\
-d_{s_{0} T}\end{array}$ & $\begin{array}{c}-a_{s_{1} T}+(a- \\
d_{T}\end{array}$ & $\begin{array}{c}a_{T} \\
-d_{s_{0} T}\end{array}$ & $\begin{array}{c}-a_{T}+(a-p) \\
-d_{T}\end{array}$ \\
\hline
\end{tabular}

Furthermore recall that $b_{T} b_{s_{0} T}=-a_{T}^{2}+(a-p) a_{T}+a p$ and $e_{s_{1} T} e_{T}=1-d_{T}^{2}$. Using these values, we can simplify the expansion of

$$
\left(\left(x_{1} t_{s_{1}} x_{1} t_{s_{1}}+x_{1} t_{s_{1}}\right)-\left(t_{s_{1}} x_{1} t_{s_{1}} x_{1}+t_{s_{1}} x_{1}\right)\right) v_{T}
$$

to find that the coefficients of $v_{S}$ vanish for $S=T, s_{0} T, s_{1} T, s_{0} s_{1} T, s_{1} s_{0} T, s_{0} s_{1} s_{0} T$, and $s_{1} s_{0} s_{1} T$. The remaining term,

$$
\begin{aligned}
& \left(\left[x_{1}\right]_{s_{1} T, s_{0} s_{1} T}\left[x_{1}\right]_{s_{1} s_{0} s_{1} T, s_{0} s_{1} s_{0} s_{1} T}\left[t_{1}\right]_{T, s_{1} T}\left[t_{1}\right]_{s_{0} s_{1} T, s_{1} s_{0} s_{1} T}\right. \\
& \left.\quad-\left[x_{1}\right]_{T, s_{0} T}\left[x_{1}\right]_{s_{1} s_{0} T, s_{0} s_{1} s_{0} T}\left[t_{1}\right]_{s_{0} T, s_{1} s_{0} T}\left[t_{1}\right]_{s_{0} s_{1} s_{0} T, s_{1} s_{0} s_{1} s_{0} T}\right) v_{s_{0} s_{1} s_{0} s_{1} T},
\end{aligned}
$$

cannot be reduced using the determined values, and so we add the assumption that this coefficient is 0 .

This concludes Part 1 , showing that $\mathscr{H}^{\lambda}$ is a $\mathscr{H}_{k}^{\text {ext }}$-module.

Part 2: $\mathscr{H}^{\lambda}$ is simple. We first show that any nontrivial submodule of $\mathscr{H}^{\lambda}$ contains some basis element $v_{T}$. We then prove that any basis element $v_{T}$ generates $\mathscr{H}^{\lambda}$, and conclude that $\mathscr{H}^{\lambda}$ contains no nontrivial proper submodules.

Claim i. If $0 \neq v \in \mathscr{H}^{\lambda}$, then $\mathscr{H}_{k}^{\text {ext }} v$ contains some element of the basis $v_{T}$.

Proof. For any $S \in \mathcal{T}_{\lambda}$, let

$$
W_{S}=\left(w_{1}-c_{S}(1)\right)^{2}+\left(w_{2}-c_{S}(2)\right)^{2}+\cdots+\left(w_{k}-c_{S}(k)\right)^{2} .
$$

By Lemma 5.1,

$$
W_{S} v_{T}=\left(\sum_{i=1}^{k}\left(c_{T}(i)-c_{S}(i)\right)^{2}\right) v_{T}=0 \quad \text { if and only if } T=S .
$$

Therefore, if

$$
\operatorname{Pr}_{T}=\prod_{\substack{S \in \mathcal{I}_{\lambda} \\ S \neq T}}\left(\frac{W_{S}}{\sum_{i=1}^{k}\left(c_{T}(i)-c_{S}(i)\right)^{2}}\right), \quad \text { then } \operatorname{Pr}_{T} v_{S}=\delta_{S T} v_{T}
$$


Write $v=\sum_{S \in \mathcal{T}_{\lambda}} d_{S} v_{S}$ and $d_{S} \in \mathbb{C}$. Since $v \neq 0$, there is some $d_{T} \neq 0$, and so $v_{T}=\left(1 / d_{T}\right) \operatorname{Pr}_{T} v \in \mathscr{H}_{k}^{\text {ext }} v$.

If $c_{T}(1) \neq \pm \frac{1}{2}((a+p) \pm(b+q))$, then $\left[x_{1}\right]_{T, s_{0} T} \neq 0$. Define the operator $\sigma_{0}$ on the basis $\left\{v_{T}\right\}_{T \in \mathcal{T}_{\lambda}}$ of $\mathscr{H}^{\lambda}$ by

$$
\sigma_{0} v_{T}= \begin{cases}0 & \text { if } c_{T}(1)= \pm \frac{1}{2}((a+p) \pm(b+q)) \\ \frac{1}{\left[x_{1}\right]_{T, s_{0} T}}\left(x_{1}-\left[x_{1}\right]_{T, T}\right) v_{T} & \text { otherwise }\end{cases}
$$

and extend linearly. Though $\sigma_{0}$ is not formally an element of $\mathscr{H}_{k}^{\text {ext }}$, it defines an operator on $\mathscr{H}^{\lambda}$ via $\mathcal{H}_{k}^{\text {ext }}$, that is, $\sigma_{0} v_{T} \in \mathcal{H}_{k}^{\text {ext }} v_{T}$. Therefore if $v_{s_{0} T}$ exists, then

$$
\begin{aligned}
\sigma_{0} v_{T} & =\frac{1}{\left[x_{1}\right]_{T, s_{0} T}}\left(x_{1}-\left[x_{1}\right]_{T, T}\right) v_{T} \\
& =\frac{1}{\left[x_{1}\right]_{T, s_{0} T}}\left(\left[x_{1}\right]_{T, T} v_{T}+\left[x_{1}\right]_{T, s_{0} T} v_{s_{0} T}-\left[x_{1}\right]_{T, T} v_{T}\right)=v_{s_{0} T},
\end{aligned}
$$

and so $v_{s_{0} T} \in \mathscr{H}_{k}^{\text {ext }} v_{T}$.

Similarly, if $c_{T}(i+1) \neq c_{T}(i) \pm 1$, then $\left[t_{i}\right]_{T, s_{i} T} \neq 0$. Define the operators $\sigma_{i}$ for $i=1, \ldots, k-1$ on the basis $\left\{v_{T}\right\}_{T \in \mathcal{T}_{\lambda}}$ of $\mathscr{H}^{\lambda}$ by

$$
\sigma_{i} v_{T}= \begin{cases}0 & \text { if } c_{T}(i+1)=c_{T}(i) \pm 1, \\ \frac{1}{\left[t_{i}\right]_{T, s_{i} T}}\left(t_{s_{i}}-\left[t_{i}\right]_{T, T}\right) v_{T} & \text { otherwise }\end{cases}
$$

and extend linearly. Again, $\sigma_{i}$ is not formally an element of $\mathcal{H}_{k}^{\text {ext }}$, but rather defines an operator on $\mathscr{H}^{\lambda}$ via $\mathscr{H}_{k}^{\text {ext }}$. So if $v_{s_{i} T}$ exists, we have

$$
\begin{aligned}
\sigma_{i} v_{T} & =\frac{1}{\left[t_{i}\right]_{T, s_{i} T}}\left(t_{s_{i}}-\left[t_{i}\right]_{T, T}\right) v_{T} \\
& =\frac{1}{\left[t_{i}\right]_{T, s_{i} T}}\left(\left[t_{i}\right]_{T, T} v_{T}+\left[t_{i}\right]_{T, s_{i} T} v_{s_{i} T}-\left[t_{i}\right]_{T, T} v_{T}\right)=v_{s_{i} T},
\end{aligned}
$$

and so $v_{s_{i} T} \in \mathscr{H}_{k}^{\text {ext }} v_{T}$.

Recall from Section 4.3 that we can view every tableau either as a sequence of partitions, as we have been doing, or as a skew shape filled with integers $1, \ldots, k$ with increasing rows and columns. Viewing $T$ as a standard filling now, consider the placement of labels $i$ and $i+1$. If they are adjacent (in row or column), then $c_{T}(i+1)=c_{T}(i) \pm 1$, and so $s_{i} T$ does not exist. However, if labels $i$ and $i+1$ are nonadjacent, then $s_{i} T$ is gotten from $T$ by switching $i$ and $i+1$. For example,

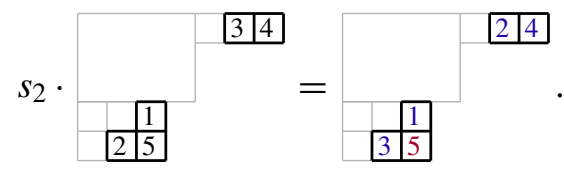


Define the tableau row $(T)$ as the filling of $\lambda / T^{(0)}$ built by placing values $1, \ldots, k$ left to right, top to bottom, consecutively (this tableau only depends on the shape of the first and last partitions in $T$ ).

Claim ii. For any tableau $T \in \mathscr{T}_{\lambda}$ and any submodule $U \subseteq \mathscr{H}^{\lambda}$,

$$
v_{T} \in U \quad \text { if and only if } \quad v_{\operatorname{row}(T)} \in U .
$$

Proof. For any $T$, the following process allows us to construct row $(T)$ by applying a series of $s_{i}$ moves to $T$ :

Reading left to right, top to bottom, find the first box that has a different filling from $\operatorname{row}(T)$. Let $j$ be the filling in this box and $i$ be the box immediately before it.

Notice $j-1$ is not placed in any boxes north (east or west) or directly west of $j$, since those boxes are filled with $1, \ldots, i$. Therefore, $j-1$ and $j$ can be switched by applying $s_{j-1}$.

If $s_{j-1} T=\operatorname{row}(T)$, we are done. Otherwise, begin again at step 1 with $s_{j-1} T$. Let $\mathrm{w}=s_{i_{\ell}} \cdots s_{i_{2}} s_{i_{1}}$ be the word generated by this process (where $s_{i_{1}}$ is the first transposition applied, and so on). In the example begun in (5-15), this process unfolds as follows.

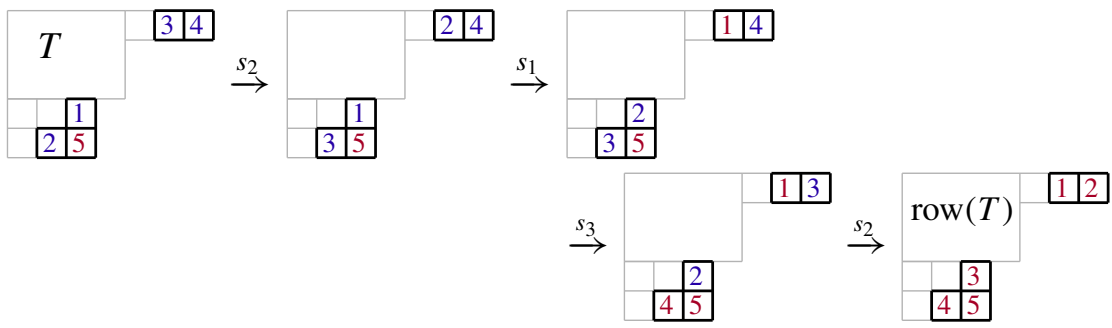

So $\mathrm{w}=s_{2} s_{3} s_{1} s_{2}$ and $s_{2} s_{3} s_{1} s_{2} T=\operatorname{row}(T)$.

If $\mathrm{w} T=s_{i_{\ell}} \cdots s_{i_{2}} s_{i_{1}} T=\operatorname{row}(T)$, then $\sigma_{i_{\ell}} \ldots \sigma_{i_{2}} \sigma_{i_{1}} v_{T}=v_{\operatorname{row}(T)}$ and so $v_{\operatorname{row}(T)} \in$ $\mathscr{H}_{k}^{\text {ext }} v_{T}$. We can apply the same process to find $\mathrm{w}^{-1} \operatorname{row}(T)=T$, implying that $\sigma_{i_{1}} \sigma_{i_{2}} \ldots \sigma_{i_{\ell}} v_{\text {row }(T)}=v_{T}$ and so $v_{T} \in \mathcal{H}_{k}^{\operatorname{ext}} v_{\operatorname{row}(T)}$.

Recall from Lemma 4.5 that if $\mu \in \mathscr{P}$, then $\mathscr{B}_{\mu}$ is the set of boxes in rows $p+1$ and below in $\mu$. If $\lambda$ is a partition containing $\mu$, let $\mathscr{R}_{\mu}^{\lambda}$ be the set of boxes $(i, j)$ in $\mathscr{B}_{\mu}$ for which box $(a+b+1-i, p+q+1-j)$ is also in $\lambda$. The criteria in (4-1) imply that the shape obtained by moving each of the boxes $(i, j) \in \mathscr{B}_{\mu}^{\lambda}$ to their complementary position $(a+b+1-i, p+q+1-j)$ gives another partition in $\mathscr{P}$, which we denote $(\lambda / \mu)^{\max }$. For example, if
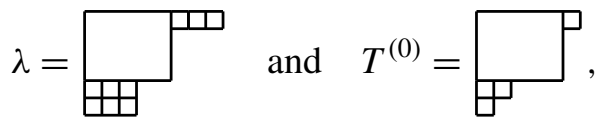

$$
\text { then } \quad \mathscr{B}_{T^{(0)}}^{\lambda}=\{(1, p+2)\} \quad \text { and } \quad(\lambda / \mu)^{\max }=\square .
$$


Moreover, since $(\lambda / \mu)^{\max } \in \lambda$, there is a tableau $S=\left((\lambda / \mu)^{\max }=S^{(0)} \searrow \cdots \searrow\right.$ $\left.S^{(k)}=\lambda\right)$ from $(\lambda / \mu)^{\max }$ to $\lambda$. Define $T_{\lambda}=\operatorname{row}(S)$, that is, $T_{\lambda}$ is the unique tableau in $\mathscr{T}_{\lambda}$ with $T^{(0)}$ highest in lexicographical order and with fillings reading left to right, top to bottom. From the example in (5-17), $T_{\lambda}$ is the last tableau pictured below in (5-18).

Claim iii. For any tableau $T \in \mathscr{T}_{\lambda}$ and submodule $U \subseteq \mathscr{H}^{\lambda}$,

$$
v_{T} \in U \quad \text { if and only if } \quad v_{T_{\lambda}} \in U \text {. }
$$

Proof. The following process allows us to construct $T_{\lambda}$ from $T$ through a series of $s_{i}$ :

(1) Use the process in Claim ii to move $T$ to $T^{\prime}=\operatorname{row}(T)$.

(2) Reading left to right, top to bottom, find the last box $(i, j)$ in $\mathscr{B}_{T^{\prime}(0)}^{\lambda}$.

(3) The box in position $(a+b+1-i, p+q+1-j)$ is filled with a 1 . Therefore, we can construct a new tableau $S=\left(S^{(0)} \searrow \cdots \searrow S^{(k)}=\lambda\right) \in \mathscr{T}_{\lambda}$, where $S^{(0)}$ is built from $T^{\prime(0)}$ by moving box $(a+b+1-i, p+q+1-j)$ to $(i, j)$, and $S^{(i)}=T^{\prime(i)}$ for $i=1, \ldots, k$. The resulting filling will have a 1 in box $(i, j)$ and $2, \ldots, k$ identical to $T^{\prime}$. This new tableau $S$ is equal to $s_{0} T^{\prime}$ (see the description of (5-3)).

(4) Use the process in Claim 2 to move to $\operatorname{row}\left(s_{0} T^{\prime}\right)$.

(5) If row $\left(s_{0} T^{\prime}\right)=T_{\lambda}$, then we are done. If not, return to step 1 with $\operatorname{row}\left(s_{0} T^{\prime}\right)$.

Let $\mathrm{w}=s_{i_{\ell}} \cdots s_{i_{2}} s_{i_{1}}$ be the word generated by this process (where $s_{i_{1}}$ is the first transposition applied, and so on). Continuing from the example in (5-16) this process proceeds as follows.

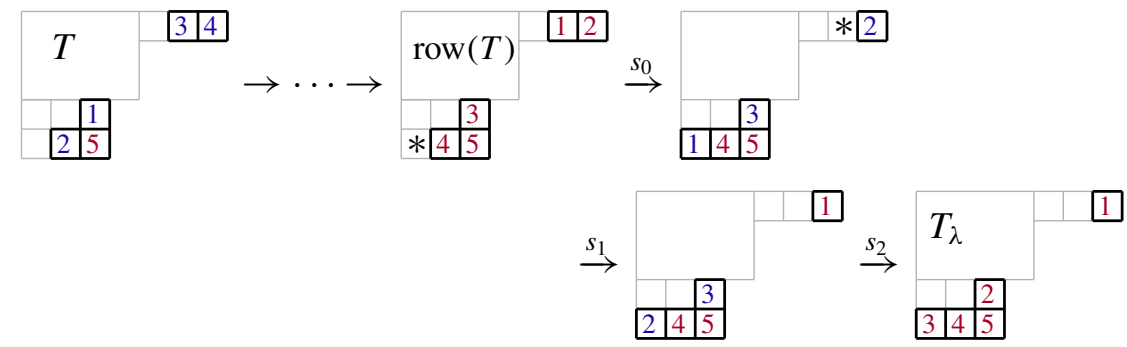

So $\mathrm{w}=s_{2} s_{1} s_{0} s_{2} s_{3} s_{1} s_{2}$, and $\mathrm{w} T=T_{\lambda}$.

If $\mathrm{w} T=s_{i_{\ell}} \cdots s_{i_{2}} s_{i_{1}} T=T_{\lambda}$, then $\sigma_{i_{\ell}} \cdots \sigma_{i_{2}} \sigma_{i_{1}} v_{T}=v_{T_{\lambda}}$ and so $v_{T^{\lambda}} \in \mathcal{H}_{k}^{\text {ext }} v_{T}$. We can apply the same process to find $\mathrm{w}^{-1} T^{\lambda}=s_{i_{1}} s_{i_{2}} \cdots s_{i_{\ell}} T_{\lambda}=T$, implying $\sigma_{i_{1}} \sigma_{i_{2}} \cdots \sigma_{i_{\ell}} v_{T_{\lambda}}=v_{T}$ and so $v_{T} \in \mathscr{H}_{k}^{\text {ext }} v_{T_{\lambda}}$.

By Claim i, any nonzero submodule $U \subseteq \mathscr{H}^{\lambda}$ contains some basis vector $v_{T}$. By Claim iii, $U$ therefore contains $v_{T_{\lambda}}$, and consequently contains all basis vectors $v_{T}$ 
of $\mathscr{H}^{\lambda}$. Thus, $U=\mathscr{H}^{\lambda}$ and so $\mathscr{H}^{\lambda}$ is simple. This concludes part 2 , and therefore completes the proof of Proposition 5.2.

Remark 5.4. We have shown slightly more than was stated in Proposition 5.2. Namely, if $\mathscr{H}^{\lambda}$ is a $\mathscr{H}_{k}^{\text {ext }}$-module with basis indexed by $T \in \mathscr{T}_{\lambda}$ and $w_{i} v_{T}=c_{T}(i) v_{T}$ for $0=1, \ldots, k$, then

(1) $t_{s_{i}} v_{T}=\left[t_{i}\right]_{T, T} v_{T}+\left[t_{i}\right]_{T, s_{i} T} v_{s_{i} T}$ and $x_{1} v_{T}=\left[x_{1}\right]_{T, T} v_{T}+\left[x_{1}\right]_{T, s_{0} T} v_{s_{0} T}$, where $\left[t_{i}\right]_{T, s_{i} T}=0$ if and only if $c_{T}(i)=c_{T}(i+1) \pm 1$, and $\left[x_{1}\right]_{T, s_{0} T}=0$ if and only if $c_{T}(1)=\frac{1}{2}( \pm(a+p) \pm(b+q))$,

(2) $\left[x_{1}\right]_{T, S}$ and $\left[t_{i}\right]_{T, S}$ satisfy items (1)-(6) of Proposition 5.2, and

(3) $\mathscr{H}^{\lambda}$ is simple as an $\mathscr{H}_{k}^{\text {ext }}$-module.

What is more is that the proof that $\mathscr{H}^{\lambda}$ is simple (part 2) relies only on the action of $\mathscr{H}_{k}$, and so $\operatorname{Res}_{\mathscr{H}_{k}^{k}}^{\mathscr{H}_{k}^{\text {ext }}}\left(\mathscr{H}^{\lambda}\right)$ is simple.

Corollary 5.5. In the setting of Theorem 4.10,

$$
\operatorname{Res}_{\Phi^{\prime}\left(\mathscr{H}_{k}^{\text {ext }}\right)}^{\operatorname{End}_{\mathfrak{m}}\left(M \otimes N \otimes V^{\otimes k}\right)}\left(\mathscr{L}^{\mu}\right) \quad \text { and } \quad \operatorname{Res}_{\Phi^{\prime}\left(\mathscr{H}_{k}\right)}^{\operatorname{End}_{\mathfrak{g}}\left(M \otimes N \otimes V^{\otimes k}\right)}\left(\mathscr{L}^{\mu}\right)
$$

are simple $\mathscr{H}_{k}^{\text {ext }}$ - and $\mathscr{H}_{k}$-modules, respectively.

Proof. By Theorem 4.10 any simple $\operatorname{End}_{\mathfrak{g}}\left(M \otimes N \otimes V^{\otimes k}\right)$-module

$$
\mathscr{L}^{\mu} \subseteq M \otimes N \otimes V^{\otimes k}
$$

has basis $\left\{v_{T} \mid T \in \mathscr{T}_{\mu}\right\}$ on which $w_{i}$ acts via $\Phi^{\prime}$ by $w_{i} v_{T}=c_{T}(i) v_{T}$. The restatement of Proposition 5.2 in Remark 5.4 implies $\mathscr{L}^{\mu}$ is simple as both a $\mathscr{H}_{k}^{\text {ext }}$-module and a $\mathscr{H}_{k}$-module.

\section{Acknowledgments}

This paper is a revised and streamlined version of my thesis [Daugherty 2010]. I would like to thank, among others, Georgia Benkart, Matthew Davis, and especially Arun Ram for many helpful conversations. I would also like to thank the referee for very thorough and thoughtful guidance.

\section{References}

[Arakawa and Suzuki 1998] T. Arakawa and T. Suzuki, "Duality between $\mathfrak{s l}_{n}(\mathbb{C})$ and the degenerate affine Hecke algebra”, J. Algebra 209:1 (1998), 288-304. MR 99h:17005 Zbl 0919.17005

[Ariki et al. 2006] S. Ariki, A. Mathas, and H. Rui, "Cyclotomic Nazarov-Wenzl algebras", Nagoya Math. J. 182 (2006), 47-134. MR 2007d:20005 Zbl 1159.20008

[Brauer 1937] R. Brauer, "On algebras which are connected with the semisimple continuous groups", Ann. of Math. (2) 38:4 (1937), 857-872. MR 1503378 Zbl 0017.39105

[Daugherty 2010] Z. Daugherty, Degenerate two-boundary centralizer algebras, thesis, University of Wisconsin, Madison, WI, 2010, available at http://tinyurl.com/Daugherty-thesis. MR 2814007 
[Daugherty et al. 2010] Z. Daugherty, A. Ram, and R. Virk, "Representation theory-The category of modules: Tantalizers", 2010, available at http://ms.unimelb.edu.au/ ram/notes.html.

[Daugherty et al. 2011] Z. Daugherty, A. Ram, and R. Virk, "Affine and degenerate affine BMW algebras: The center", preprint, 2011. arXiv 1105.4207

[Gier and Nichols 2009] J. de Gier and A. Nichols, "The two-boundary Temperley-Lieb algebra", J. Algebra 321:4 (2009), 1132-1167. MR 2009m:20005 Zbl 1188.20005

[Goodman and Wallach 1998] R. Goodman and N. R. Wallach, Representations and invariants of the classical groups, Encyclopedia of Mathematics and its Applications 68, Cambridge University Press, Cambridge, 1998. MR 99b:20073 Zbl 0901.22001

[Jacobson 1962] N. Jacobson, Lie algebras, Interscience Tracts in Pure and Applied Mathematics 10, Interscience/Wiley, New York, 1962. MR 26\#1345 Zbl 0121.27504

[Macdonald 1995] I. G. Macdonald, Symmetric functions and Hall polynomials, 2nd ed., Clarendon, Oxford, 1995. MR 96h:05207 Zbl 0824.05059

[Nazarov 1996] M. Nazarov, "Young's orthogonal form for Brauer's centralizer algebra", J. Algebra 182:3 (1996), 664-693. MR 97m:20057 Zbl 0868.20012

[Okada 1998] S. Okada, "Applications of minor summation formulas to rectangular-shaped representations of classical groups", J. Algebra 205:2 (1998), 337-367. MR 99g:20081 Zbl 0915.20023

[Okounkov and Vershik 1996] A. Okounkov and A. Vershik, "A new approach to representation theory of symmetric groups”, Selecta Math. (N.S.) 2 (1996), 581-605. MR 99g:20024 Zbl 0959.20014

[Orellana and Ram 2007] R. Orellana and A. Ram, "Affine braids, Markov traces and the category O", pp. 423-473 in Algebraic groups and homogeneous spaces (Mumbai, 2004), edited by V. B. Mehta, Tata Inst. Fund. Res. Stud. Math. 19, Narosa, New Delhi, 2007. MR 2008m:17034 Zbl 1172.17009

[Stanley 1986] R. P. Stanley, "Symmetries of plane partitions", J. Combin. Theory Ser. A 43:1 (1986), 103-113. MR 87m:05017a Zbl 0602.05007

Received May 2, 2011. Revised August 22, 2011.

\section{ZAJJ DAUGHERTY}

DEPARTMENT OF MATHEMATICS

DARTMOUTH COLLEGE

6188 KEMENY HALL

HANOVER, NH 03755

UNITED STATES

zajj.b.daugherty@dartmouth.edu

http://www.math.dartmouth.edu/ zdaugherty 


\title{
PACIFIC JOURNAL OF MATHEMATICS
}

\author{
http://pacificmath.org \\ Founded in 1951 by \\ E. F. Beckenbach (1906-1982) and F. Wolf (1904-1989)
}

\section{EDITORS}

V. S. Varadarajan (Managing Editor)

Department of Mathematics

University of California

Los Angeles, CA 90095-1555

pacific@math.ucla.edu

Vyjayanthi Chari

Department of Mathematics

University of California

Riverside, CA 92521-0135

chari@math.ucr.edu

\section{Robert Finn}

Department of Mathematics Stanford University

Stanford, CA 94305-2125

finn@math.stanford.edu

Kefeng Liu

Department of Mathematics

University of California

Los Angeles, CA 90095-1555

liu@math.ucla.edu
Darren Long

Department of Mathematics

University of California

Santa Barbara, CA 93106-3080

long@math.ucsb.edu

Jiang-Hua Lu

Department of Mathematics

The University of Hong Kong

Pokfulam Rd., Hong Kong jhlu@maths.hku.hk

Alexander Merkurjev

Department of Mathematics

University of California

Los Angeles, CA 90095-1555

merkurev@math.ucla.edu
Sorin Popa

Department of Mathematics University of California

Los Angeles, CA 90095-1555 popa@math.ucla.edu

Jie Qing

Department of Mathematics

University of California

Santa Cruz, CA 95064

qing@cats.ucsc.edu

Jonathan Rogawski

Department of Mathematics

University of California

Los Angeles, CA 90095-1555

jonr@math.ucla.edu

\section{PRODUCTION}

pacific@math.berkeley.edu

\section{SUPPORTING INSTITUTIONS}

ACADEMIA SINICA, TAIPEI

CALIFORNIA INST. OF TECHNOLOGY INST. DE MATEMÁTICA PURA E APLICADA KEIO UNIVERSITY

MATH. SCIENCES RESEARCH INSTITUTE NEW MEXICO STATE UNIV.

OREGON STATE UNIV.

\author{
STANFORD UNIVERSITY \\ UNIV. OF BRITISH COLUMBIA \\ UNIV. OF CALIFORNIA, BERKELEY \\ UNIV. OF CALIFORNIA, DAVIS \\ UNIV. OF CALIFORNIA, LOS ANGELES \\ UNIV. OF CALIFORNIA, RIVERSIDE \\ UNIV. OF CALIFORNIA, SAN DIEGO \\ UNIV. OF CALIF., SANTA BARBARA
}

\author{
UNIV. OF CALIF., SANTA CRUZ \\ UNIV. OF MONTANA \\ UNIV. OF OREGON \\ UNIV. OF SOUTHERN CALIFORNIA \\ UNIV. OF UTAH \\ UNIV. OF WASHINGTON \\ WASHINGTON STATE UNIVERSITY
}

These supporting institutions contribute to the cost of publication of this Journal, but they are not owners or publishers and have no responsibility for its contents or policies.

See inside back cover or pacificmath.org for submission instructions.

The subscription price for 2012 is US \$420/year for the electronic version, and \$485/year for print and electronic.

Subscriptions, requests for back issues from the last three years and changes of subscribers address should be sent to Pacific Journal of Mathematics, P.O. Box 4163, Berkeley, CA 94704-0163, U.S.A. Prior back issues are obtainable from Periodicals Service Company, 11 Main Street, Germantown, NY 12526-5635. The Pacific Journal of Mathematics is indexed by Mathematical Reviews, Zentralblatt MATH, PASCAL CNRS Index, Referativnyi Zhurnal, Current Mathematical Publications and the Science Citation Index.

The Pacific Journal of Mathematics (ISSN 0030-8730) at the University of California, c/o Department of Mathematics, 969 Evans Hall, Berkeley, CA 94720-3840, is published monthly except July and August. Periodical rate postage paid at Berkeley, CA 94704, and additional mailing offices. POSTMASTER: send address changes to Pacific Journal of Mathematics, P.O. Box 4163, Berkeley, CA 94704-0163.

PJM peer review and production are managed by EditFLOW ${ }^{\mathrm{TM}}$ from Mathematical Sciences Publishers.

PUBLISHED BY PACIFIC JOURNAL OF MATHEMATICS

at the University of California, Berkeley 94720-3840

A NON-PROFIT CORPORATION

Typeset in LATEX

Copyright $(02012$ by Pacific Journal of Mathematics 


\section{PACIFIC JOURNAL OF MATHEMATICS}

Volume $258 \quad$ No. $1 \quad$ July 2012

On the complexity of sails

LUKAS BRANTNER

Construction of Lagrangian submanifolds in $\mathbb{C P}^{n}$

QING Chen, SEN Hu and XiaOweI XU

Semisimple tunnels

SANGBUM CHO and DARRYL MCCULLOUGH

Degenerate two-boundary centralizer algebras

\section{ZAJJ DAUGHERTY}

Heegaard genera in congruence towers of hyperbolic 3-manifolds

BOGWANG JEON

The Heisenberg ultrahyperbolic equation: The basic solutions as distributions

ANTHONY C. KABLE

Rational Seifert surfaces in Seifert fibered spaces

JOAN E. LiCATA and JoshuA M. SABLOFF

Delaunay cells for arrangements of flats in hyperbolic space 